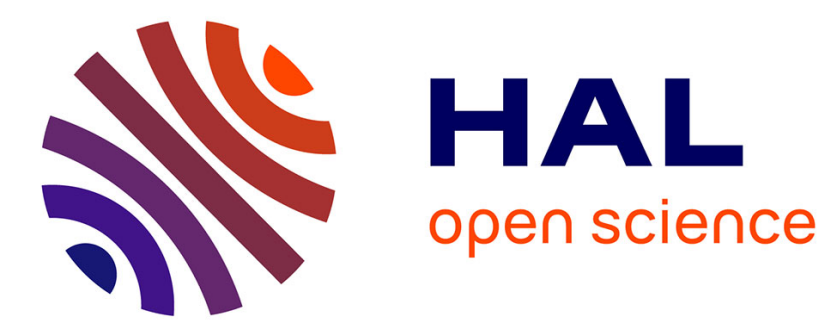

\title{
Supersingular irreducible symplectic varieties
}

\author{
Lie $\mathrm{Fu}$, Zhiyuan $\mathrm{Li}$
}

\section{To cite this version:}

Lie Fu, Zhiyuan Li. Supersingular irreducible symplectic varieties. 2019. hal-02158586

\section{HAL Id: hal-02158586 \\ https://hal.science/hal-02158586}

Preprint submitted on 18 Jun 2019

HAL is a multi-disciplinary open access archive for the deposit and dissemination of scientific research documents, whether they are published or not. The documents may come from teaching and research institutions in France or abroad, or from public or private research centers.
L'archive ouverte pluridisciplinaire HAL, est destinée au dépôt et à la diffusion de documents scientifiques de niveau recherche, publiés ou non, émanant des établissements d'enseignement et de recherche français ou étrangers, des laboratoires publics ou privés. 


\title{
SUPERSINGULAR IRREDUCIBLE SYMPLECTIC VARIETIES
}

\author{
LIE FU AND ZHIYUAN LI
}

\begin{abstract}
In complex geometry, irreducible symplectic varieties, also known as compact hyper-Kähler varieties, are natural higher-dimensional generalizations of K3 surfaces. We propose to study such varieties defined over fields of positive characteristics, especially the supersingular ones, generalizing the theory of supersingular K3 surfaces. In this work, we are mainly interested in the following two types of irreducible symplectic varieties over an algebraically closed field of characteristic $p>0$, under natural numerical conditions :

(1) smooth moduli spaces of stable sheaves on K3 surfaces and

(2) smooth Albanese fibers of moduli spaces of sheaves on abelian surfaces. Several natural definitions of the supersingularity for irreducible symplectic varieties are discussed, which are proved to be equivalent in both cases (1) and (2). Their equivalence is conjectured in general. On the geometric aspect, we conjecture that the unirationality characterizes the supersingularity for irreducible symplectic varieties, motivated from the fact that supersingular K3 surfaces are all unirational. We establish the equivalence between the unirationality and the supersingularity in case (1) and the equivalence between the rational chain connectedness and the supersingularity in case (2). On the motivic aspect, contrary to the situation over the complex numbers, we conjecture that the algebraic cycles on supersingular irreducible symplectic varieties, as well as their intersection theory, should be as simple as possible: its Chow motive is of supersingular abelian type, the rational Griffiths group vanishes, the algebraically trivial part of the rational Chow group has a supersingular abelian variety as its algebraic representative à la Murre, and the rational Chow ring satisfies Beauville's multiplicative splitting property which takes a particularly simple form. As evidences, we prove all these conjectures on algebraic cycles for supersingular varieties in both cases (1) and (2).
\end{abstract}

Date: November 16, 2018.

2010 Mathematics Subject Classification. 14J28, 53C26, 14J60, 14C25, 14M20, 14C15, 14K99.

Key words and phrases. K3 surfaces, irreducible symplectic varieties, hyper-Kähler varieties, supersingularity, moduli spaces, unirationality, motives, Bloch-Beilinson conjecture, Beauville splitting conjecture.

Lie $\mathrm{Fu}$ is supported by the Agence Nationale de la Recherche through ECOVA (ANR-15CE40-0002), HodgeFun (ANR-16-CE40-0011) and LABEX MILYON (ANR-10-LABX-0070) of Université de Lyon. Lie $\mathrm{Fu}$ is also benefited from Projet Inter-Laboratoire 2017 and 2018 by Fédération de Recherche en Mathématiques Rhône-Alpes/Auvergne CNRS 3490. Zhiyuan Li is supported by National Science Fund for General Program (11771086), Key Program (11731004); the "Dawn" Program (17SG01) of Shanghai Education Commission. 


\section{Contents}

1. Introduction 3

1.1. Supersingular K3 surfaces 3

1.2. Irreducible symplectic varieties 4

1.3. A legion of conjectures 4

1.4. Main results 8

2. Generalities on the notion of supersingularity 10

2.1. Supersingularity via $F$-crystals $\quad 10$

2.2. Supersingularity via cycle class map 11

2.3. Full supersingularity and algebraic cycles $\quad 12$

2.4. Supersingular abelian varieties and their motives 13

$\begin{array}{ll}3 . & \text { Supersingular irreducible symplectic varieties } \\ \text { 3. } & 17\end{array}$

$\begin{array}{ll}\text { 3.1. Irreducible symplectic varieties } & 18\end{array}$

3.2. Artin supersingularity and formal Brauer group 18

3.3. Artin supersingularity vs. Shioda supersingularity 20

3.4. Unirationality vs. supersingularity 21

3.5. Algebraic cycles and supersingularity 22

3.6. Birational irreducible symplectic varieties 25

4. Moduli spaces of stable sheaves on K3 surfaces 26

4.1. Preliminaries on K3 surfaces over positive characteristic 26

4.2. Moduli spaces of stable sheaves 28

4.3. Moduli spaces of sheaves on supersingular K3 surfaces 30

4.4. Hilbert schemes and their birational models 33

4.5. Relating moduli spaces to Hilbert schemes 34

5. Moduli spaces of twisted sheaves on K3 surfaces 38

5.1. Twisted sheaves on K3 surfaces 38

5.2. Moduli spaces of twisted sheaves 39

5.3. From moduli spaces of twisted sheaves to those of untwisted ones $\quad 40$

6. Generalized Kummer varieties 42

6.1. Preliminaries on abelian surfaces over positive characteristic $\quad 42$

6.2. Generalized Kummer varieties 44

6.3. Supersingularity and abelian motive $\quad 45$

6.4. Rational chain connectedness 46

6.5. Chow rings of supersingular generalized Kummer varieties 47

7. Irreducible symplectic varieties of generalized Kummer type 47

7.1. Moduli spaces of stable sheaves on abelian surfaces 47

7.2. Main results for generalized Kummer type moduli spaces 49

7.3. A remark on the unirationality 51

References $\quad 52$ 


\section{INTRODUCTION}

This paper is an attempt to generalize to higher dimensions the beautiful theory and exciting progresses on supersingular K3 surfaces. Throughout the paper, let $k$ be an algebraically closed field of characteristic $p>0$ and denote by $W(k)$ its ring of Witt vectors.

1.1. Supersingular K3 surfaces. Recall that a K3 surface is a smooth projective surface defined over $k$ with trivial canonical bundle and vanishing first cohomology of the structure sheaf. It takes a central place in the classification of algebraic surfaces. Let $X$ be a K3 surface. On one hand, $X$ is called Artin supersingular if its formal Brauer group $\widehat{\operatorname{Br}}(X)$ is the formal additive group $\widehat{\mathbb{G}}_{a}$ (see [5], [6] or $\S 3.2$ ), or equivalently, the Newton polygon associated to the second crystalline cohomology $H_{\text {cris }}^{2}(X / W(k))$ is a straight line (of slope 1 ). On the other hand, Shioda [81] has introduced another notion of supersingularity for K3 surfaces by considering the algebraicity of the $\ell$-adic cohomology classes of degree 2 , for any $\ell \neq p$ : we say that $X$ is Shioda supersingular if the first Chern class map

$$
c_{1}: \operatorname{Pic}(X) \otimes \mathbb{Q}_{\ell} \rightarrow H_{e ́ t}^{2}\left(X, \mathbb{Q}_{\ell}(1)\right)
$$

is surjective; this condition is independent of $\ell$, as it is equivalent to the maximality of the Picard rank, i.e. $\rho_{X}=b_{2}(X)=22$. It is easy to see that Shioda supersingularity implies Artin supersingularity. Conversely, the Tate conjecture [83] for K3 surfaces over finite fields, solved in [66], [67], [59], [18], [55], [19] and [43], implies that these two notions actually coincide for any algebraically closed fields of positive characteristic, $c f$. [54, Theorem 4.8]:

$$
\text { Shioda supersingularity } \Leftrightarrow \text { Artin supersingularity. }
$$

The supersingularity being essentially a cohomological notion, it is natural to look for its relation to geometric properties. Unlike complex K3 surfaces, there exist unirational K3 surfaces over fields of positive characteristic, first examples being constructed by Shioda in [81] and Rudakov-Šafarevič in [77]; then Artin [5] and Shioda [81] observed that unirational K3 surfaces must have maximal Picard rank 22, hence are supersingular. Conversely, one expects that the unirationality is a geometric characterization of the supersingularity for K3 surfaces:

Conjecture 1.1 (Artin [5], Shioda [81], Rudakov-Šafarevič [77]). A K3 surface is supersingular if and only if it is unirational.

This conjecture has been confirmed over fields of characteristic 2 by RudakovŠafarevič [77] via the existence of quasi-elliptic fibration. In [53], Liedtke proved the conjecture when the characteristic is at least 5 by using moving torsors. Very recently, Bragg and Lieblich [16] proved the conjecture for all positive characteristics, based on the study of their twistor spaces of supersingular K3 surfaces. Let us also remark that the unirationality of supersingular K3 surfaces implies that the Chow motive is of Tate type and in particular that the Chow group of 0-cycles is isomorphic to $\mathbb{Z}$, thus contrasting drastically to the situation over the complex numbers, where $\mathrm{CH}_{0}$ is infinite dimensional by Mumford's celebrated observation in $[63]$. 
1.2. Irreducible symplectic varieties. Thanks to the progress mentioned above, our knowledge for supersingular K3 surfaces is quite satisfying. We want to investigate the higher dimensional analogues. In the setting of complex geometry, the natural generalizations of K3 surfaces are the irreducible holomorphic symplectic manifolds or equivalently, compact hyper-Kähler manifolds. Those are by definition the simply connected compact Kähler manifolds admitting a Ricci flat metric with the holonomy group being the compact symplectic group. Together with abelian varieties (or more generally complex tori) and Calabi-Yau varieties, they form the fundamental building blocks for compact Kähler manifolds with vanishing first Chern class, thanks to the Beauville-Bogomolov decomposition theorem [11, Théorème 2]. Irreducible symplectic varieties over the complex numbers are extensively studied from various points of view over the last three decades. We refer to the huge existing literature for more details [11], [39], [35, Part III].

In positive characteristic, there seems no commonly accepted definition of irreducible symplectic varieties (see however [19]). In this paper, we define them as smooth projective varieties $X$ defined over $k$ with trivial étale fundamental group and $E_{1}$-degenerate Frölicher spectral sequence, such that $H^{0}\left(X, \Omega_{X / k}^{2}\right)$ is generated by a symplectic (i.e. nowhere-degenerate) algebraic 2-form (Definition 3.1).

The objective of this paper is to initiate a systematic study of supersingular irreducible symplectic varieties: we will discuss several natural definitions of the supersingularity, propose some general conjectures and provide ample evidence for them.

The notion(s) of supersingularity, which is subtle for higher-dimensional varieties, can be approached in essentially two ways ( $c f . \S 2)$ : via formal groups and $F$-crystal structures on the crystalline cohomology as Artin did [5], or via the algebraicity of $\ell$-adic or crystalline cohomology groups as Shioda did [81]. More precisely, an irreducible symplectic variety $X$ is called

- $2^{\text {nd }}$-Artin supersingular, if the $F$-crystal $H_{\text {cris }}^{2}(X / W(k))$ is supersingular, that is, its Newton polygon is a straight line. Artin supersingularity turns out to be equivalent to the condition that the formal Brauer group $\widehat{\operatorname{Br}}(X)$ is isomorphic to $\widehat{\mathbb{G}}_{a}$, provided that it is formally smooth of dimension 1 (Proposition 3.8).

- $2^{\text {nd }}$-Shioda supersingular, if its Picard rank $\rho(X)$ is equal to its second Betti number $b_{2}(X)$. Or equivalently, the first Chern class map is surjective for the $\ell$-adic cohomology for any $\ell \neq p$.

The reason that we use the second cohomology in both approaches is the general belief that for an irreducible symplectic variety, its second cohomology endowed with its enriched structure should capture a significant part of the information of the variety up to the birational equivalence.

1.3. A legion of conjectures. Inspired by the results for K3 surfaces discussed before, we expect the following conjectural picture for irreducible symplectic varieties, generalizing the equivalence (1) as well as Conjecture 1.1.

Equivalence Conjecture. An irreducible symplectic variety is $2^{\text {nd }}$-Artin supersingular if and only if it is $2^{\text {nd }}$-Shioda supersingular.

This is a consequence of the crystalline Tate conjecture (cf. [61]). 
We actually make the stronger Conjecture 3.13 , which says that for any $2^{n d}$ Artin supersingular irreducible symplectic variety $X$, the other cohomology groups satisfy similar properties (see Definitions 2.2 and 2.4):

- (Fully Artin supersingular.) For any $0 \leq i \leq 2 \operatorname{dim}(X)$, the $F$-crystal $H_{\text {cris }}^{i}(X / W(k))$ is supersingular, i.e. its Newton polygon is a straight line.

- (Fully Shioda supersingular.)

- For any $0 \leq i \leq \operatorname{dim}(X)$, the crystalline cycle class map $\mathrm{CH}^{i}(X)_{K} \rightarrow$ $H_{\text {cris }}^{2 i}(X / W(k))_{K}$ is surjective, where $K$ is the field of fractions of $W(k)$.

- For any $1 \leq i \leq \operatorname{dim}(X)$, there exists a supersingular abelian variety $A b^{i}$ defined over $k$ such that we have an algebraic correspondence between $X$ and $A b^{i}$ inducing an isomorphism of $F$-crystals between (the $(i-1)$-th Tate twist of) $H_{\text {cris }}^{2 i-1}(X / W(k))$ and $H_{\text {cris }}^{1}\left(A b^{i} / W(k)\right)$.

- For any $i$ and any $\ell \neq p$, the $\ell$-adic cycle class map $\mathrm{CH}^{i}(X) \otimes \mathbb{Q}_{\ell} \rightarrow$ $H_{e t}^{2 i}\left(X, \mathbb{Q}_{\ell}\right)$ is surjective.

As a geometric characterization for the cohomological notion of supersingularity, we propose the following:

Unirationality Conjecture. An irreducible symplectic variety is $2^{\text {nd }}$-Artin supersingular if and only if it is unirational.

The previous conjecture has the following weaker version.

RCC Conjecture. An irreducible symplectic variety is $2^{\text {nd }}$-Artin supersingular if and only if it is rationally chain connected.

We also expect that the algebraic cycles on a supersingular irreducible symplectic variety are "as easy as possible". The most fundamental way to formulate this is in the category of motives:

Abelian Motive Conjecture. The rational Chow motive of a $2^{\text {nd }}$-Artin supersingular irreducible symplectic variety is a direct summand of the motive of a supersingular abelian variety.

By the work of Fakhruddin [27], having supersingular abelian motive implies that the variety is fully Shioda supersingular (cf. Corollary 2.16). Therefore to summarize, we expect that the notions in the following diagram of implications are all equivalent for irreducible symplectic varieties:

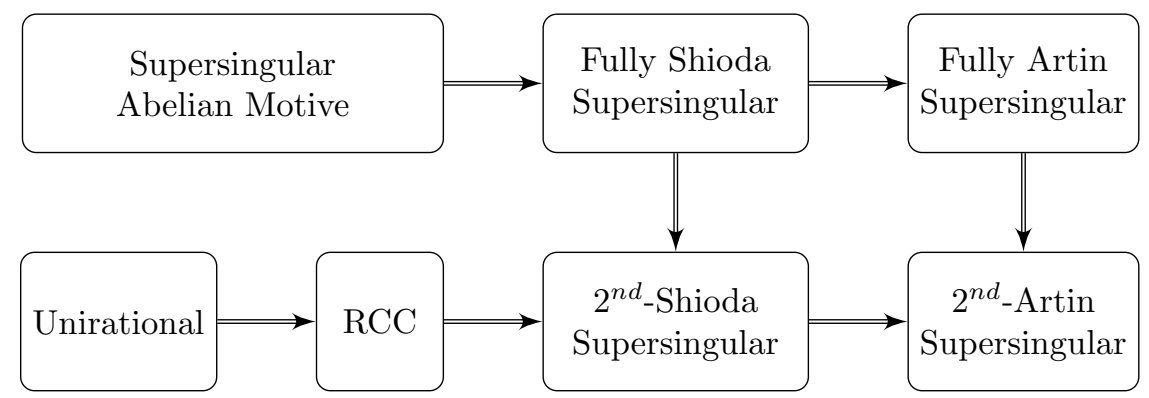

Figure 1. Characterizations of supersingularity for ISV 
As a consequence of the Abelian Motive Conjecture, using [27], we have the following supersingular version of the Bloch-Beilinson conjecture, which gives a quite complete description of the additive structure of the rational Chow groups of supersingular irreducible symplectic varieties. Recall that $\mathrm{CH}^{i}(X)_{\text {alg }}$ is the subgroup of the Chow group $\mathrm{CH}^{i}(X)$ consisting of algebraically trivial cycles. Denote also by $\overline{\mathrm{CH}}^{i}(X)_{\mathbb{Q}}$ the $i^{\text {th }}$ rational Chow group modulo the numerical equivalence.

Supersingular Bloch-Beilinson Conjecture. Let $X$ be a supersingular irreducible symplectic variety. Then for any $0 \leq i \leq \operatorname{dim}(X)$,

- The numerical equivalence and the algebraic equivalence coincide on $\mathrm{CH}^{i}(X)_{\mathbb{Q}}$. In particular, the Griffiths group $\operatorname{Griff}^{i}(X)_{\mathbb{Q}}=0$.

- There exists a regular surjective homomorphism

$$
\nu_{i}: \mathrm{CH}^{i}(X)_{\mathrm{alg}} \rightarrow A b^{i}(X)
$$

to an abelian variety $A b^{i}(X)$, called the algebraic representative, such that it is universal for regular homomorphisms from $\mathrm{CH}^{i}(X)$ alg to abelian varieties.

- The kernel of $\nu_{i}$ is finite and $A b^{i}(X)$ is a supersingular abelian variety of dimension $\frac{1}{2} b_{2 i-1}(X)$.

- The intersection product restricted to the subring $\mathrm{CH}^{*}(X)_{\text {alg }}$ is zero.

In particular, the kernel of the algebra epimorphism $\mathrm{CH}^{*}(X)_{\mathbb{Q}} \rightarrow \overline{\mathrm{CH}}^{*}(X)_{\mathbb{Q}}$ is a square zero graded ideal given by the supersingular abelian varieties $A b^{*}(X)_{\mathbb{Q}}:=$ $\oplus_{i} A b^{i}(X) \otimes_{\mathbb{Z}} \mathbb{Q}$.

Beyond the context of irreducible symplectic varieties, we believe that all the conclusions in the previous conjecture hold more generally for any so-called fully Shioda supersingular variety (Definition 2.4), see the Fully Shioda Supersingular Bloch-Beilinson Conjecture 2.11.

Now for irreducible symplectic varieties, the rational Chow ring has a supplementary feature, namely there is Beauville's insight that the Bloch-Beilinson filtration should possess a canonical multiplicative splitting [13], [87]. Inspired by this Splitting Conjecture, in a recent joint work of the first author and Charles Vial [30], the following Section Conjecture is proposed and studied mainly over the complex numbers. We expect it to hold in general in any characteristic (see Conjecture 3.17), but let us state it only in the supersingular situation here.

Supersingular Section Conjecture. Let $X$ be a supersingular irreducible symplectic variety. Then for any $0 \leq i \leq \operatorname{dim}(X)$, there is a subspace $\mathrm{DCH}^{i}(X) \subset$ $\mathrm{CH}^{i}(X)_{\mathbb{Q}}$, whose elements are called distinguished cycles, such that

- the composition $\mathrm{DCH}^{i}(X) \hookrightarrow \mathrm{CH}^{i}(X)_{\mathbb{Q}} \rightarrow \overline{\mathrm{CH}}^{i}(X)_{\mathbb{Q}}$ is an isomorphism.

- $\mathrm{DCH}^{*}(X):=\bigoplus_{i} \mathrm{DCH}^{i}(X)$ forms a $\mathbb{Q}$-subalgebra of $\mathrm{CH}^{*}(X)_{\mathbb{Q}}$.

- The Chern class $c_{i}\left(T_{X}\right) \in \mathrm{DCH}^{i}(X)$ for any $i$.

In other words, there exists a section of the natural algebra epimorphism $\mathrm{CH}^{*}(X)_{\mathbb{Q}} \rightarrow$ $\overline{\mathrm{CH}}^{*}(X)_{\mathbb{Q}}$ whose image contains all Chern classes of $X$.

The key point is that the section is multiplicative, which cannot hold in general for other fully supersingular varieties. The section in the conjecture is expected 
to be canonical. Combining the Equivalence Conjecture, the Supersingular BlochBeilinson Conjecture and the Section Conjecture, we have the following rather complete conjectural description for the structure of the rational Chow ring of a supersingular irreducible symplectic variety, which qualifies as the supersingular version of Beauville's Splitting Conjecture [13]:

Supersingular Beauville Splitting Conjecture. Let $X$ be a supersingular irreducible symplectic variety. Then the rational Chow ring of $X$ has a multiplicative decomposition

$$
\mathrm{CH}^{*}(X)_{\mathbb{Q}}=\mathrm{DCH}^{*}(X) \oplus \mathrm{CH}^{*}(X)_{\mathrm{alg}, \mathbb{Q}}
$$

such that

- $\mathrm{DCH}^{*}(X)$ is a graded $\mathbb{Q}$-subalgebra containing all the Chern classes of $X$ and mapped isomorphically to $\overline{\mathrm{CH}}^{*}(X)_{\mathbb{Q}}$ via the natural projection. It also provides a $\mathbb{Q}$-structure for the $\ell$-adic even cohomology ring $H_{e ̂ t}^{2 *}\left(X, \mathbb{Q}_{\ell}\right)$ for all $\ell \neq p$, i.e. the restriction of the cycle class map $\operatorname{DCH}^{*}(X)_{\mathbb{Q}_{\ell}} \stackrel{\stackrel{\sim}{\rightarrow}}{\rightarrow}$ $H^{2 *}\left(X, \mathbb{Q}_{\ell}\right)$ is an isomorphism.

- The algebraically trivial cycles $\mathrm{CH}^{*}(X)_{a l g, \mathbb{Q}}$ form a square zero graded ideal, which is mapped isomorphically to supersingular abelian varieties given by the algebraic representatives $A b^{*}(X)_{\mathbb{Q}}$.

In other words, $\mathrm{CH}^{*}(X)_{\mathbb{Q}}$ is the square zero extension of a graded subalgebra isomorphic to $\overline{\mathrm{CH}}^{*}(X)_{\mathbb{Q}}$ by a graded module $A b^{*}(X)_{\mathbb{Q}}$.

Remark 1.2. The decomposition (2) is expected to be canonical. Moreover, the $\overline{\mathrm{CH}}^{*}(X)_{\mathbb{Q}}$-module structure on $A b^{*}(X)_{\mathbb{Q}}$ should be determined by, or at least closely related to, the $H^{2 *}(X)$-module structure on $H^{2 *-1}(X)$, where $H$ is some Weil cohomology theory.

To summarize:

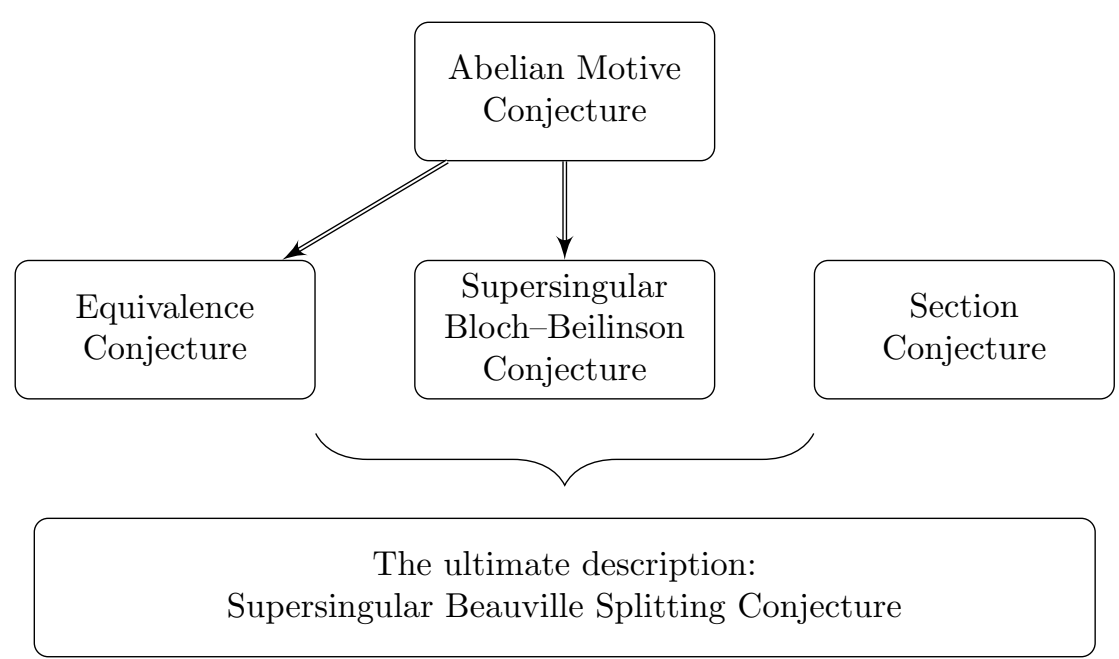

Figure 2. Conjectures on algebraic cycles of supersingular ISV 
1.4. Main results. As in characteristic zero, two important families of examples of irreducible symplectic varieties are provided by the moduli spaces of stable sheaves on K3 surfaces and the Albanese fibers of the moduli spaces of stable sheaves on abelian surfaces. As evidences to the aforementioned conjectures, we establish them for most of the varieties in these two series.

For moduli spaces of sheaves on K3 surfaces, the key result is the following Theorem 1.3, which relates these moduli spaces birationally to punctual Hilbert schemes of K3 surfaces, in the supersingular situation.

Theorem 1.3. Let $k$ be an algebraically closed field of characteristic $p>0$ and $S$ be a K3 surface defined over $k$. Let $H$ be an ample line bundle on $S$ and $X$ the moduli space of $H$-semistable sheaves on $S$ with Mukai vector $v=\left(r, c_{1}, s\right)$ satisfying $\langle v, v\rangle \geq 0$ and $r>0$.

(1) If $H$ is general with respect to $v$, then $X$ is an irreducible symplectic variety of dimension $2 n=\langle v, v\rangle+2$ and deformation equivalent to the $n^{\text {th }}$ Hilbert scheme of points of $S$. Moreover, $X$ is $2^{\text {nd }}$-Artin supersingular if and only if $S$ is supersingular.

(2) If $S$ is supersingular and $v$ is coprime to $p$, then $X$ is a $2^{\text {nd }}$-Artin supersingular irreducible symplectic variety and it is birational to the Hilbert scheme $S^{[n]}$, where $n=\frac{\langle v, v\rangle+2}{2}$.

The notion of generality for $H$ and that of $v$ being coprime to $p$ are explained in Definitions 4.4 and 4.7 respectively. In fact, these conditions are the natural ones for $X$ to be smooth.

The birational equivalence in the theorem above can be chosen to be the composition of several birational equivalences (between liftable irreducible symplectic varieties) that are liftable to characteristic zero bases, see Theorem 4.16. Combining this with an analysis of the motives of Hilbert schemes of supersingular K3 surfaces (Proposition 4.13), we can prove all the conjectures in $\S 1.3$ for the moduli spaces considered in Theorem 1.3:

Corollary 1.4. The notation and assumption are as in Theorem 1.3. If $v$ is coprime to $p$ and $H$ is general with respect to $v$, then the following conditions are equivalent:

(i) $S$ is supersingular.

(ii) $X$ is $2^{\text {nd }}$-Artin supersingular.

(ii') $X$ is fully Artin supersingular.

(iii) $X$ is $2^{\text {nd }}$-Shioda supersingular.

(iii') $X$ is fully Shioda supersingular.

(iv) $X$ is unirational.

(v) The Chow motive of $X$ is of Tate type (i.e. a direct sum of Tate motives).

In the case that the above conditions hold, the cycle class maps induce isomorphisms $\mathrm{CH}^{*}(X)_{K} \simeq H_{\text {cris }}^{*}(X / W)_{K}$ and $\mathrm{CH}^{*}(X)_{\mathbb{Q}_{\ell}} \simeq H_{\text {ét }}^{*}\left(X, \mathbb{Q}_{\ell}\right)$ for all $\ell \neq p$.

Similarly, for irreducible symplectic varieties of generalized Kummer type, we have the following results.

Theorem 1.5. Let $k$ be an algebraically closed field of characteristic $p>0$. Let $A$ be an abelian surface defined over $k$. Let $H$ be an ample line bundle on $A$ and $X$ the Albanese fiber of the projective moduli space of $H$-stable sheaves on $A$ with Mukai vector $v=\left(r, c_{1}, s\right)$ satisfying $\langle v, v\rangle \geq 2$ and $r>0$. 
(1) If $H$ is general with respect to $v$ and $X$ is smooth over $k$, then $X$ is an irreducible symplectic variety of dimension $2 n:=\langle v, v\rangle-2$ and deformation equivalent to the $n^{\text {th }}$ generalized Kummer variety. Moreover, $X$ is $2^{\text {nd }}$-Artin supersingular if and only if $A$ is supersingular.

(2) Suppose $A$ is supersingular and $p+\frac{1}{2}\langle v, v\rangle$. Then $X$ is $2^{\text {nd }}$-Artin supersingular and it is birational to the $n^{\text {th }}$ generalized Kummer variety associated to some supersingular abelian surface, with $n=\frac{\langle v, v\rangle-2}{2}$.

The numerical condition on $v$ is natural to ensure the smoothness of $X$. The birational equivalence here is again liftable to characteristic zero, see Theorem 7.6. Together with a study of rational curves and motives of generalized Kummer varieties associated to supersingular abelian varieties (Propositions 6.6, 6.8 and 6.10), we can show all the conjectures in 1.3, except the Unirationality Conjecture, for most Kummer type moduli spaces of sheaves on abelian varieties:

Corollary 1.6. The notation and assumption are as in Theorem 1.5. When $p+$ $\frac{1}{2}\langle v, v\rangle$ and $H$ is general with respect to $v$, the following conditions are equivalent:

(i) $A$ is supersingular.

(ii) $X$ is $2^{\text {nd }}$-Artin supersingular.

(ii') $X$ is fully Artin supersingular.

(iii) $X$ is $2^{\text {nd }}$-Shioda supersingular.

(iii') $X$ is fully Shioda supersingular.

(iv) $X$ is rationally chain connected.

(v) The Chow motive of $X$ is a supersingular abelian motive.

If one of these conditions are satisfied, all the conclusions in the Supersingular Bloch-Beilinson Conjecture, the Section Conjecture and the Supersingular Beauville Splitting Conjecture hold for $X$.

Remark 1.7. Some remarks on these results are in order.

- The coprime assumption on $v$ in Theorem 1.3 and Corollary 1.4 is automatically satisfied if $p+\frac{1}{2} \operatorname{dim} X-1$, see Remark 4.9.

- Our results also hold for moduli spaces of twisted sheaves on supersingular K3 surfaces with Artin invariant at most 9. See Theorem 5.9 in $\S 5$. We believe that this method also works for twisted sheaves on the product of supersingular elliptic curves. We leave the details to readers.

- The unirationality for generalized Kummer type moduli spaces seems out of reach in higher dimensions. We do not know how to decide the unirationality on a single example even in the case of generalized Kummer fourfolds associated to the product of two supersingular elliptic curves.

- It turns out that the understanding of motives of supersingular abelian varieties are crucial in the study of the algebraic cycles on supersingular irreducible symplectic varieties. For this reason, we obtain in passing Theorem 2.13 , a result possibly known to experts, which computes the rational Chow motive of a supersingular abelian variety $A$. Roughly speaking, $\mathfrak{h}^{i}(A)$ is a Tate motive when $i$ is even; $\mathfrak{h}^{i}(A)$ is a direct sum of copies of $\mathfrak{h}^{1}(E)\left(-\frac{i-1}{2}\right)$ when $i$ is odd, where $E$ is a supersingular elliptic curve. Based on this computation, we are able to recover and strengthen in Corollary 2.16 Fakhruddin's result in [27] on varieties with supersingular abelian motives. 
Conventions: Throughout this paper, $k$ is an algebraically closed field of characteristic $p>0, W=W(k)$ is its ring of Witt vectors, $W_{i}=W / p^{i} W$ is the $i$-th truncated Witt ring of $k$, and $K=K(W)=W[1 / p]$ is the field of fractions of $W$. If $X$ is a variety defined over a field $F$ and let $L$ be a field extension of $F$, we write $X_{L}=X \otimes_{F} L$ for the base change.

Acknowledgement: The authors want to thank Nicolas Addington, François Charles, Cyril Demarche, Najmuddin Fakhruddin, Daniel Huybrechts, Qizheng Yin and Weizhe Zheng for very helpful discussions. Lie Fu is also grateful to the Hausdorff Research Institute for Mathematics for the hospitality during the 2017 Trimester K-theory and related fields.

\section{Generalities on the nOtion OF SUPERsingularity}

In this section, we introduce several notions of supersingularity for cohomology groups of algebraic varieties in general and discuss the relations between them.

2.1. Supersingularity via $F$-crystals. Let $X$ be a smooth projective variety of dimension $n$ defined over $k$. The ring of Witt vectors $W=W(k)$ comes equipped with a morphism $\sigma: W \rightarrow W$ induced by the Frobenius morphism $x \mapsto x^{p}$ of $k$. For any $i \in \mathbb{N}$, we denote by $H_{\text {cris }}^{i}(X / W)$ the $i$-th integral crystalline cohomology of $X$, which is a $W$-module whose rank is equal to the $i$-th Betti number ${ }^{1}$ of $X$. We set

$$
H^{i}(X):=H_{\text {cris }}^{i}(X / W) / \text { torsion, } H^{i}(X)_{K}=H_{\text {cris }}^{i}(X / W) \otimes_{W} K .
$$

Then $H^{i}(X)$ is a free $W$-module and it is endowed with a natural $\sigma$-linear map

$$
\varphi: H^{i}(X) \rightarrow H^{i}(X)
$$

induced from the absolute Frobenius morphism $F: X \rightarrow X$ by functoriality. Moreover, by Poincaré duality, $\varphi$ is injective.

The pair $\left(H^{i}(X), \varphi\right)$ (resp. $\left.\left(H^{i}(X)_{K}, \varphi_{K}\right)\right)$ forms therefore an $F$-crystal (resp. $F$ isocrystal), associated to which we have the Newton polygon $\mathrm{Nt}^{i}(X)$ and the Hodge polygon $\operatorname{Hdg}^{i}(X)$. According to Dieudonné-Manin's classification theorem (cf. [56]), the $F$-crystal $\left(H^{i}(X), \varphi\right)$ is uniquely determined, up to isogeny, by (the slopes with multiplicities of) the Newton polygon $\mathrm{Nt}^{i}(X)$. Following [56]:

Definition 2.1. We say that an $F$-crystal $(M, \varphi)$ is ordinary if its Newton polygon and Hodge polygon agree and supersingular if its Newton polygon is a straight line.

For an $F$-crystal $\left(H^{2 r}(X), \varphi\right)$ arising as the even-degree crystalline cohomology of a projective variety $X$, the supersingularity condition that $\mathrm{Nt}^{2 r}(X)$ is a straight line implies that all slopes of $\mathrm{Nt}^{2 r}(X)$ are equal to $r$, since the Frobenius action on the image of an algebraic cycle of codimension $r$ is the multiplication by $p^{r}$.

Definition 2.2 (Artin supersingularity). For a given integer $i$, a smooth projective variety $X$ over $k$ is called $i^{\text {th }}$-Artin supersingular, if the $F$-crystal $\left(H^{i}(X), \varphi\right)$ is supersingular. $X$ is called fully Artin supersingular if it is $i^{\text {th }}$-Artin supersingular for all $i$.

\footnotetext{
${ }^{1}$ By definition, the $i$-th Betti number of $X$, denoted by $b_{i}(X)$, is the dimension (over $\mathbb{Q}_{\ell}$ ) of the $\ell$-adic cohomology group $H_{e ́ t}^{i}\left(X, \mathbb{Q}_{\ell}\right)$. The Betti number $b_{i}(X)$ is independent of the choice of the prime number $\ell$ different from $p$ ([23], [24], [41]).
} 
Example 2.3 (Supersingular abelian varieties). Let $A$ be an abelian variety over $k$. We say that $A$ is supersingular if the $F$-crystal $\left(H^{1}(A), \varphi\right)$ is supersingular, i.e. $A$ is $1^{\text {st }}$-Artin supersingular. When $g=1$, this definition is equivalent to the classical notion of supersingularity for elliptic curves: for instance, the group of $p$-torsion points is trivial. We will show in $\S 2.4$ that a supersingular abelian variety is actually fully Artin supersingular.

2.2. Supersingularity via cycle class map. We discuss the supersingularity in the sense of Shioda. Let $X$ be a smooth projective variety defined over $k$. For any $r \in \mathbb{N}$, there is the crystalline cycle class map

$$
\mathfrak{c l}^{r}: \mathrm{CH}^{r}(X) \otimes_{\mathbb{Z}} K \longrightarrow H^{2 r}(X)_{K}:=H_{\text {cris }}^{2 r}(X / W) \otimes_{W} K .
$$

whose image lands in the eigen-space of eigenvalue $p^{r}$ with respect to the action of $\varphi$ on $H^{2 r}(X)$,

Definition 2.4 (Shioda supersingularity). A smooth projective variety $X$ defined over $k$ is called

- $(2 r)^{t h}$-Shioda supersingular, if (3) is surjective;

- even Shioda supersingular ${ }^{2}$ if (3) is surjective for all $r$;

- $(2 r+1)^{t h}$-Shioda supersingular, if there exist a supersingular abelian variety $A$ and an algebraic correspondence $\Gamma \in \mathrm{CH}^{\operatorname{dim} X-r}(X \times A)$ such that the cohomological correspondence $\Gamma_{*}: H_{\text {cris }}^{2 r+1}(X / W)_{K} \rightarrow H_{\text {cris }}^{1}(A / W)_{K}$ is an isomorphism;

- odd Shioda supersingular, if it is $(2 r+1)^{t h}$-Shioda supersingular for all $r$;

- fully Shioda supersingular, if it is even and odd Shioda supersingular.

Remark 2.5 ("Shioda implies Artin"). Each notion of Shioda supersingularity is stronger than the corresponding notion of Artin supersingularity. More precisely,

(i) For even-degree cohomology, the $(2 r)^{t h}$-Shioda supersingularity implies that the Frobenius action $\varphi$ is the multiplication by $p^{r}$ on the crystalline cohomology of degree $2 r$, hence we have the $(2 r)^{t h}$-Artin supersingularity (i.e. the $F$-crystal $\left(H^{2 r}(X), \varphi\right)$ is supersingular). The converse is implied by the crystalline Tate conjecture (cf. [3, 7.3.3.2]).

(ii) For odd-degree cohomology, the idea of Shioda supersingularity is "of niveau 1 " and supersingular. The implication from $(2 r+1)^{t h}$-Shioda supersingularity to $(2 r+1)^{t h}$-Artin supersingularity follows from the definition and Example 2.3. The converse follows again from the crystalline Tate conjecture.

(iii) Moreover, one can try to define the (even) Shioda supersingularity using other Weil cohomology theory. For instance, for any prime number $\ell$ different from $p$, let $H^{i}\left(X, \mathbb{Q}_{\ell}(r)\right)$ be the $\ell$-adic cohomology of $X$ and let

$$
\mathfrak{c r}_{\ell}^{r}: \mathrm{CH}^{r}(X) \otimes \mathbb{Q}_{\ell} \rightarrow H^{2 r}\left(X, \mathbb{Q}_{\ell}(r)\right) .
$$

be the $\ell$-adic cycle class map. Note that the standard conjecture implies that the surjectivities of $\mathfrak{c l}_{\ell}^{r}$ and $\mathfrak{c l}^{r}$ are equivalent for all $\ell \neq p$, as the images of the cycle class maps for various Weil cohomology theories share the same $\mathbb{Q}$ structure, namely $\overline{\mathrm{CH}}^{r}(X)_{\mathbb{Q}}$, the rational Chow group modulo the numerical equivalence.

\footnotetext{
${ }^{2}$ This is called fully rigged in [84, Definition 5.4]
} 
When $r=1$, the $2^{n d}$-Shioda supersingularity does not depend on the choice of the Weil cohomology theory, as it is equivalent to say that the Picard rank is maximal: $\operatorname{rank}(\operatorname{Pic}(X))=b_{2}(X)$.

Example 2.6 (Abelian varieties). We have mentioned in Example 2.3 that supersingular abelian varieties are fully Artin supersingular. We will see in $§ 2.4$, Theorem 2.13 that supersingular abelian varieties are actually fully Shioda supersingular.

Remark 2.7 (First supersingularity). For a smooth projective variety, the $1^{s t}$ Shioda supersingularity and the $1^{\text {st }}$-Artin supersingularity are equivalent, as they are both equivalent to the supersingularity of the Picard variety (or Albanese variety).

One can get many examples of $2^{n d}$-Shioda supersingular varieties from varieties with "sufficiently many" rational curves. The result below, which says that the rational chain connectedness implies the algebraicity of $H^{2}$, is well-known in characteristic 0 , and it holds in positive characteristics as well.

Theorem 2.8 ( $c f$. [34, Theorem 1.2]). Let $X$ be smooth projective variety over $k$. If $X$ is rationally chain connected, then the first Chern class map induces an isomorphism $\operatorname{Pic}(X) \otimes \mathbb{Q}_{\ell} \cong H_{e}^{2}\left(X, \mathbb{Q}_{\ell}(1)\right)$ for all $\ell \neq p$. In particular, $\rho(X)=b_{2}(X)$ and $X$ is $2^{\text {nd }}$-Shioda supersingular.

2.3. Full supersingularity and algebraic cycles. We explore in this subsection the conjectural implications on the Chow groups of the full (Artin or Shioda) supersingularity discussed in $\S 2.1$ and $\S 2.2$.

Let $X$ be a smooth projective variety that is fully Shioda supersingular (Definition 2.4), which should be equivalent to full Artin supersingularity under the generalized crystalline Tate conjecture. Then by definition,

- All the even cohomology groups are of niveau 0 (i.e. of Tate type). Equivalently, $H^{2 i}(X)$ is of coniveau $i$ for all $0 \leq i \leq \operatorname{dim} X$.

- All the odd cohomology groups are of niveau 1 (i.e. of abelian type). Equivalently, $H^{2 i+1}(X)$ is of coniveau $\geq i$ for all $0 \leq i \leq \operatorname{dim} X-1$.

In particular, for any $2 \leq j \leq i \leq \operatorname{dim} X$, the coniveau of $H^{2 i-j}(X)$ is at least $i-j+1$. By the general philosophy of coniveau, the conjectural Bloch-Beilinson filtration $F^{*}$ on the rational Chow group satisfies that ( $c f .[3, \S 11.2]$, [85, Conjecture 23.21])

$$
G r_{F}^{j} \mathrm{CH}^{i}(X)_{\mathbb{Q}}=0, \forall j \geq 2, \forall i \text {. }
$$

Therefore by the conjectural separatedness of the filtration $F$, one expects that $F^{2} \mathrm{CH}^{i}(X)_{\mathbb{Q}}=0$ in this case. It is generally believed that $F^{2}$ is closely related, if not equal, to the Abel-Jacobi kernel. Hence one can naturally conjecture that for fully supersingular varieties, all rational Chow groups are representable ([63]), or better, their homologically trivial parts are represented by abelian varieties. The precise statement is Conjecture 2.11 below. Before that, let us explain the notion of representability with some more details.

Recall first the notion of algebraic representatives ([9], [64]) for Chow groups, developed by Murre. Let $\mathrm{CH}^{*}(X)_{\text {alg }}$ be the group of algebraically trivial cycles modulo the rational equivalence.

Definition 2.9 (Regular homomorphism [64, Definition 1.6.1]). Let $X$ be a smooth projective variety and $A$ an abelian variety. A homomorphism $\phi: \mathrm{CH}^{i}(X)_{\text {alg }} \rightarrow A$ 
is called regular, if for any family of algebraic cycles, that is, a connected pointed variety $\left(T, t_{0}\right)$ together with a cycle $Z \in \mathrm{CH}^{i}(X \times T)$, the map

$$
\begin{aligned}
T & \rightarrow A \\
t & \mapsto \phi\left(Z_{t}-Z_{t_{0}}\right)
\end{aligned}
$$

is a morphism of algebraic varieties.

Definition 2.10 (Algebraic representative [64]). Let $X$ be a smooth projective variety and $i$ be an integer. An algebraic representative for cycles of codimension $i$, is a couple $\left(\nu_{i}, A b^{i}\right)$, where $A b^{i}$ is an abelian variety and $\nu_{i}: \mathrm{CH}^{i}(X)_{\text {alg }} \rightarrow A b^{i}$ is a regular homomorphism (Definition 2.9), and it is universal in the following sense: for any regular homomorphism to an abelian variety $\phi: \mathrm{CH}^{i}(X)_{\text {alg }} \rightarrow A$, there exists a unique homomorphism of abelian varieties $\bar{\phi}: A b^{i} \rightarrow A$ such that $\phi=\bar{\phi} \circ \nu_{i}$. It is easy to see that an algebraic representative, if exists, is unique up to unique isomorphism and $\nu_{i}$ is surjective $([64, \S 1.8])$.

As examples, the algebraic representative for the Chow group of divisors (resp. 0cycles) is the Picard variety (resp. the Albanese variety). The main result of [64] is the existence of an algebraic representative for codimension 2 cycles and its relation to the algebraic part of the intermediate Jacobian. However, the understanding of the kernel of $\nu_{i}$, when it is not zero, seems out of reach.

In the spirit of the Bloch-Beilinson Conjecture, we can now make the following speculation on the algebraic cycles on fully supersingular varieties:

Conjecture 2.11 (Fully supersingular Bloch-Beilinson Conjecture). Let $X$ be a smooth projective variety over $k$ which is fully Shioda supersingular. Then for any $0 \leq i \leq \operatorname{dim}(X)$,

- The numerical equivalence and the algebraic equivalence coincide on $\mathrm{CH}^{i}(X)_{\mathbb{Q}}$. In particular, the rational Griffiths group $\operatorname{Griff}^{i}(X)_{\mathbb{Q}}=0$.

- There exists a regular surjective homomorphism

$$
\nu_{i}: \mathrm{CH}^{i}(X) \mathrm{alg} \rightarrow A b^{i}(X)
$$

to an abelian variety $A b^{i}(X)$, which is the algebraic representative in the sense of Murre (Definition 2.10).

- The kernel of $\nu_{i}$ is finite and $\operatorname{dim} A b^{i}(X)=\frac{1}{2} b_{2 i-1}(X)$.

- $A b^{i}$ is a supersingular abelian variety.

- The intersection product restricted to $\mathrm{CH}^{*}(X)_{\text {alg }}$ is zero.

In particular, the kernel of the algebra epimorphism $\mathrm{CH}^{*}(X)_{\mathbb{Q}} \rightarrow \overline{\mathrm{CH}}^{*}(X)_{\mathbb{Q}}$ is a square zero graded ideal given by the supersingular abelian varieties $A b^{*}(X)_{\mathbb{Q}}:=$ $\oplus_{i} A b^{i}(X) \otimes_{\mathbb{Z}} \mathbb{Q}$.

Thanks to the work of Fakhruddin [27], Conjecture 2.11 is known for supersingular abelian varieties as well as other varieties with supersingular abelian motives. We will give an account of this aspect in $§ 2.4$.

2.4. Supersingular abelian varieties and their motives. In this subsection, we illustrate the previous discussions in the special case of abelian varieties and establish some results on their motives for later use.

Let $A$ be a $g$-dimensional abelian variety defined over $k$. For any $n \in \mathbb{N}, H^{n}(A):=$ $H_{\text {cris }}^{n}(A / W)$ is a torsion free $W$-module of rank $\left(\begin{array}{c}2 g \\ n\end{array}\right)$ and there exists a canonical 
isomorphism of $F$-crystals induced by the cup-product

$$
H^{n}(A) \cong \bigwedge^{n} H^{1}(A) \text {. }
$$

By definition, $A$ is called supersingular if the $F$-crystal $H^{1}(A)$ is so (Example 2.3). In this case, by (4), all slopes of the $F$-crystal $\left(H^{n}(A), \varphi\right)$ are the same $\left(=\frac{n}{2}\right)$, and hence $H^{n}(A)$ is as well a supersingular $F$-crystal for all $n \in \mathbb{N}$; in other words, $A$ is fully Artin supersingular (Definition 2.2).

Before moving on to deeper results on cycles and motives of supersingular abelian varieties, let us recall some basics on their motivic decomposition. Denote by $\operatorname{CHM}(k)_{\mathbb{Q}}$ the category of rational Chow motives over $k$. Building upon earlier works of Beauville [10] and [12] on Fourier transforms of algebraic cycles of abelian varieties, Deninger-Murre [26] produced a canonical motivic decomposition for any abelian variety (actually more generally for any abelian scheme; see [45] for explicit formulae of the projectors):

$$
\mathfrak{h}(A)=\bigoplus_{i=0}^{2 g} \mathfrak{h}^{i}(A) \text { in } \operatorname{CHM}(k)_{\mathbb{Q}}
$$

such that the Beauville component $\mathrm{CH}^{i}(A)_{(s)}$ is identified with $\mathrm{CH}^{i}\left(\mathfrak{h}^{2 i-s}(A)\right)$. Furthermore, Kings [44] proved that $\mathfrak{h}^{i}(A)=\wedge^{i} \mathfrak{h}^{1}(A)$ for all $i, \mathfrak{h}^{2 g}(A) \simeq \mathbb{1}(-g)$ and $\wedge^{i} \mathfrak{h}^{1}(A)=0$ for $i>2 g$.

Lemma 2.12. Let $E$ be a supersingular elliptic curve over $k$. Then for any natural number $j$, we have in $\operatorname{CHM}(k)_{\mathbb{Q}}$,

$$
\begin{aligned}
& \operatorname{Sym}^{2 j} \mathfrak{h}^{1}(E) \simeq \mathbb{1}(-j)^{\oplus j(2 j+1)} \\
& \operatorname{Sym}^{2 j+1} \mathfrak{h}^{1}(E) \simeq \mathfrak{h}^{1}(E)(-j)^{\oplus(j+1)(2 j+1)} .
\end{aligned}
$$

Proof. As $\operatorname{dim} \operatorname{End}(E)_{\mathbb{Q}}=4$, we have $\mathfrak{h}^{1}(E) \otimes \mathfrak{h}^{1}(E) \simeq \mathbb{1}(-1)^{\oplus 4}$. Hence as a direct summand, $\operatorname{Sym}^{2} \mathfrak{h}^{1}(E)$ is also of Tate type, actually isomorphic to $\mathbb{1}(-1)^{\oplus 3}$. Since $\operatorname{Sym}^{2 j} \mathfrak{h}^{1}(E)$ is a direct summand of $\left(\operatorname{Sym}^{2} \mathfrak{h}^{1}(E)\right)^{\otimes j}=\mathbb{1}(-j)^{\oplus 3^{j}}$, it is also of the form $\mathbb{1}(-j)^{\oplus m}$. The rank can be obtained by looking at the realization. As for the odd symmetric powers, $\operatorname{Sym}^{2 j+1} \mathfrak{h}^{1}(E)$ is a direct summand of $\operatorname{Sym}^{2 j} \mathfrak{h}^{1}(E) \otimes$ $\mathfrak{h}^{1}(E)=\mathfrak{h}^{1}(E)(-j)^{\oplus j(2 j+1)}$, therefore $\operatorname{Sym}^{2 j+1} \mathfrak{h}^{1}(E)(j)$ is a direct summand of $\mathfrak{h}^{1}\left(E^{j(2 j+1)}\right)$. This corresponds to (up to isogeny) a sub-abelian variety of the supersingular abelian variety $E^{j(2 j+1)}$, which must be itself supersingular, hence isogenous to a power of $E$ by [73, Theorem 4.2]. In other words, $\operatorname{Sym}^{2 j+1} \mathfrak{h}^{1}(E)(j) \simeq$ $\mathfrak{h}^{1}\left(E^{m}\right)$, for some $m \in \mathbb{N}$, which can be identified by looking at the realization.

We can now compute the Chow motives of supersingular abelian varieties:

Theorem 2.13. Let $A$ be a g-dimensional supersingular abelian variety defined over an algebraically closed field $k$ of positive characteristic $p$. Let $b_{i}=\left(\begin{array}{c}2 g \\ i\end{array}\right)$ be the $i$-th Betti number of $A$. Then in the category $\operatorname{CHM}(k)_{\mathbb{Q}}$, we have for any $i$,

$$
\begin{aligned}
\mathfrak{h}^{2 i}(A) & \simeq \mathbb{1}(-i)^{\oplus b_{2 i}} ; \\
\mathfrak{h}^{2 i+1}(A) & \simeq \mathfrak{h}^{1}(E)(-i)^{\oplus \frac{1}{2} b_{2 i+1}},
\end{aligned}
$$

where $E$ is a/any supersingular elliptic curve. In particular, $A$ is fully Shioda supersingular. 
Proof. By Oort's result [73, Theorem 4.2], an abelian variety $A$ is supersingular if and only if it is isogenous to the self-product of a/any supersingular elliptic curve $E$. In $\operatorname{CHM}(k)_{\mathbb{Q}}$, we denote $\Lambda:=\mathbb{1}^{\oplus g}$ and then $\mathfrak{h}^{1}(A) \simeq \mathfrak{h}^{1}\left(E^{g}\right)=\mathfrak{h}^{1}(E) \otimes \Lambda$, since isogenous abelian varieties have isomorphic rational Chow motives. Standard facts on tensor operations in idempotent-complete symmetric monoïdal categories (cf. [32, Lecture 6]) yields

$$
\mathfrak{h}^{i}(A) \simeq \bigwedge^{i}\left(\mathfrak{h}^{1}(E) \otimes \Lambda\right) \simeq \bigoplus_{\lambda \rightarrow i} \mathbb{S}_{\lambda} \mathfrak{h}^{1}(E) \otimes \mathbb{S}_{\lambda^{\prime}} \Lambda,
$$

where $\lambda$ runs over all partitions of $i, \lambda^{\prime}$ is the transpose of $\lambda$ and $\mathbb{S}_{\lambda}$ is the Schur functor associated to $\lambda . \mathbb{S}_{\lambda^{\prime}} \Lambda$ being a direct sum of the unit motive $\mathbb{1}$, let us take a closer look at $\mathbb{S}_{\lambda} \mathfrak{h}^{1}(E)$. Recall that (cf. [4] for example $\left.{ }^{3}\right)$

- $\mathbb{S}_{\lambda} \mathfrak{h}^{1}(E)=0$ if $\lambda$ has length at least 3 ;

- if $\lambda$ has length at most 2 , say $\lambda=(a+b, a)$ with $a, b \geq 0$ and $2 a+b=i$, then since $\wedge^{2} \mathfrak{h}^{1}(E) \simeq \mathbb{1}(-1)$ is a $\otimes$-invertible object, we have

$$
\mathbb{S}_{(a+b, a)} \mathfrak{h}^{1}(E)=\left(\bigwedge^{2} \mathfrak{h}^{1}(E)\right)^{\otimes a} \otimes \operatorname{Sym}^{b} \mathfrak{h}^{1}(E)=\mathbb{1}(-a) \otimes \operatorname{Sym}^{b} \mathfrak{h}^{1}(E)
$$

Combining this with (7) and using Lemma 2.12, we see that $\mathfrak{h}^{i}(A)$ is a direct sum of some copies of $\mathbb{1}\left(-\frac{i}{2}\right)$ if $i$ is even and a direct sum of some copies of $\mathfrak{h}^{1}(E)\left(-\frac{i-1}{2}\right)$ if $i$ is odd. The numbers of copies needed are easily calculated by looking at their realizations.

Definition 2.14 (Supersingular abelian motives). Let $\mathrm{CHM}(k)_{\mathbb{Q}}$ be the category of rational Chow motives over $k$. Let $\mathcal{M}^{s s a b}$ be the idempotent-complete symmetric monoïdal subcategory of $\operatorname{CHM}(k)_{\mathbb{Q}}$ generated by the motives of supersingular abelian varieties. A smooth projective variety $X$ is said to have supersingular abelian motive if its rational Chow motive $\mathfrak{h}(X)$ belongs to $\mathcal{M}^{\text {ssab }}$.

Remark 2.15. $\mathcal{M}^{\text {ssab }}$ contains the Tate motives by definition. Thanks to Theorem $2.13, \mathcal{M}^{s s a b}$ is actually generated, as idempotent-complete tensor category, by the Tate motives together with $\mathfrak{h}^{1}(E)$ for a/any supersingular elliptic curve $E$. It can be shown that any object in $\mathcal{M}^{s s a b}$ is a direct summand of the motive of some supersingular abelian variety. Therefore, for a smooth projective variety $X$, the condition of having supersingular abelian motive is exactly Fakhruddin's notion of "strong supersingularity" in [27].

The following result confirms in particular the Fully Supersingular Bloch-Beilinson Conjecture 2.11 and the full Shioda supersingularity for varieties with supersingular abelian motives. The results $(i i)-(v)$ are due to Fakhruddin [27]; while our proof presented below is somehow different and emphasizes the more fundamental result Theorem 2.13; $(i)$ and $(v i)$ are new according to authors' knowledge.

Corollary 2.16 ( $c f$. Fakhruddin [27]). Let $X$ be an n-dimensional smooth projective variety defined over an algebraically closed field $k$ of characteristic $p>0$, such that $X$ has supersingular abelian motive (Definition 2.14). Let $b_{i}$ be the $i$-th Betti number of $X$. Then we have the following:

\footnotetext{
${ }^{3}$ The convention in [4] is the graded/super one, which is the reason why the symmetric product and exterior product are switched from ours when applied to an "odd" object $\mathfrak{h}^{1}$ in loc. cit. We prefer to stick to the ungraded convention so the comparison to the corresponding facts from classical cohomology theory is more transparent.
} 
(i) In the category $\operatorname{CHM}(k)_{\mathbb{Q}}$, we have

$$
\mathfrak{h}(X) \simeq \bigoplus_{i=0}^{n} \mathbb{1}(-i)^{\oplus b_{2 i}} \oplus \bigoplus_{i=0}^{n-1} \mathfrak{h}^{1}(E)(-i)^{\oplus \frac{1}{2} b_{2 i+1}},
$$

where $E$ is a supersingular elliptic curve.

(ii) $X$ is fully Shioda supersingular (Definition 2.4):

(a) For any $i$ and any prime number $\ell \neq p$, the cycle class map $\mathrm{CH}^{i}(X)_{\mathbb{Q}_{\ell}} \rightarrow$ $H_{e t}^{2 i}\left(X, \mathbb{Q}_{\ell}\right)$ is surjective.

(b) For any $i$, the cycle class map $\mathrm{CH}^{i}(X)_{K} \rightarrow H_{\text {cris }}^{2 i}(X / W)_{K}$ is surjective.

(c) For any $i$, there exist a supersingular abelian variety $B$ together with an algebraic correspondence $\Gamma \in \mathrm{CH}^{n-i}(X \times B)$ such that $\Gamma_{\star}: H_{\text {cris }}^{2 i+1}(X / W)_{K} \rightarrow$ $H_{\text {cris }}^{1}(B / W)_{K}$ is an isomorphism.

In particular, $X$ is fully Artin supersingular (Definition 2.2).

(iii) The numerical equivalence and the algebraic equivalence coincide. In particular, for any $i$, the Griffiths group is of torsion: $\operatorname{Griff}^{i}(X)_{\mathbb{Q}}=0$.

(iv) $\mathrm{CH}^{i}(X)_{\mathbb{Q}}=\mathrm{CH}^{i}(X)_{(0)} \oplus \mathrm{CH}^{i}(X)_{(1)}$ with $\mathrm{CH}^{i}(X)_{(0)} \simeq \mathbb{Q}^{\oplus b_{2 i}}$ providing a $\mathbb{Q}$ structure for cohomology and $\mathrm{CH}^{i}(X)_{(1)} \simeq E^{\frac{1}{2} b_{2 i-1}} \otimes_{\mathbb{Z}} \mathbb{Q}$ is the algebraically trivial part.

(v) $\mathrm{CH}^{i}(X)_{\mathrm{alg}}$ has an algebraic representative $\left(\nu_{i}, A b^{i}\right)$ with $\operatorname{ker}\left(\nu_{i}\right)$ finite and $A b^{i}$ a supersingular abelian variety of dimension $\frac{1}{2} b_{2 i-1}$.

(vi) The intersection product restricted to $\mathrm{CH}^{*}(X)_{\text {alg }}$ is zero.

Proof. For (i), using Theorem 2.13, the motive of $X$ must be a direct sum of Tate motives and Tate twists of $\mathfrak{h}^{1}(E)$. Then the precise numbers of Tate twists and copies are easily determined by looking at the realization.

For $(i i)$, applying various realization functors, $(a),(b)$ and $(c)$ all follow immediately from $(8)$ in $(i)$ by taking $B=E^{\frac{1}{2} b_{2 i-1}}$.

For (iii), Using $(i)$, it suffices to observe that the numerical equivalence and the algebraic equivalence are the same on elliptic curves and all the Griffiths groups of a point and an elliptic curve are trivial.

For $(i v)$, it is an immediate consequence of $(i)$ together with the simple fact that $\mathrm{CH}^{1}\left(\mathfrak{h}^{1}(E)\right)=\mathrm{CH}_{(1)}^{1}(E) \simeq \mathrm{Pic}^{0}(E) \otimes_{\mathbb{Z}} \mathbb{Q} \simeq E \otimes_{\mathbb{Z}} \mathbb{Q}$.

For $(v)$, we argue similarly as in [27]: denote $B=E^{\frac{1}{2} b_{2 i-1}}$, then in (8), the only term contributes non-trivially to $\mathrm{CH}^{i}(X)_{\text {alg }}$ is $\mathfrak{h}^{1}(E)(1-i)^{\oplus b_{2 i-1}}$. Now the isomorphisms (8) of rational Chow motives can be interpreted as follows: there exist a positive integer $N$ and two correspondences $Z_{1}, Z_{2} \in \mathrm{CH}^{n-i+1}(X \times B)$, such that the two compositions of

$$
Z_{1}^{*}: \mathrm{CH}^{1}(B)_{\mathrm{alg}}=\operatorname{Pic}^{0}(B) \rightarrow \mathrm{CH}^{i}(X)_{\mathrm{alg}}
$$

and

$$
Z_{2, *}: \mathrm{CH}^{i}(X)_{\mathrm{alg}} \rightarrow \mathrm{CH}^{1}(B)_{\mathrm{alg}}=\mathrm{Pic}^{0}(B)
$$

are both the multiplication by $N$. By the divisibility of $\mathrm{CH}^{i}(X)_{\text {alg }}$ and $\operatorname{Pic}^{0}(B)$ (cf. [15, Lemma 1.3]), both $Z_{1}^{*}$ and $Z_{2, *}$ are surjective and the kernel of $Z_{2, *}$ is finite. The surjectivity of $Z_{2, *}$ implies the representability of $\mathrm{CH}^{i}(X)_{\text {alg }}$ and in particular (by [78] for example) there exists an algebraic representative $\nu_{i}: \mathrm{CH}^{i}(X)_{\text {alg }} \rightarrow$ $A b^{i}$. By the universal property of algebraic representative, the (regular) surjective homomorphism $Z_{2, *}$ is factorized through $\nu_{i}$, hence the kernel of $\nu_{i}$ is finite and $\operatorname{Pic}^{0}(B)$ is dominated by $A b^{i}$. On the other hand, the surjectivities of $Z_{1}^{*}$ and 
$\nu_{i}$ show that $A b^{i}$ is also dominated by $\operatorname{Pic}^{0}(B)$. Therefore, $A b^{i}$ is isogenous to $\operatorname{Pic}^{0}(B)$, hence is supersingular of dimension $\frac{1}{2} b_{2 i-1}$.

Finally for $(v i)$, recall first that any algebraically trivial cycle on $X$ is a linear combination of cycles of the form $\Gamma_{*}(\alpha)$, where $\Gamma \in \mathrm{CH}(C \times X)$ is a correspondence from a connected smooth projective curve $C$ to $X$ and $\alpha$ is a 0 -cycle of degree 0 on $C$. Therefore we only need to show that for any two connected smooth projective curves $C_{1}, C_{2}, \Gamma_{i} \in \mathrm{CH}\left(C_{i} \times X\right)$ and $\alpha_{i} \in \mathrm{CH}_{0}\left(C_{i}\right)_{\operatorname{deg} 0}$ for $i=1,2$, then $\Gamma_{1, *}\left(\alpha_{1}\right) \cdot \Gamma_{2, *}\left(\alpha_{2}\right)=0$ in $\mathrm{CH}(X)$. Indeed, let $\Gamma$ be the correspondence from $C_{1} \times C_{2}$ to $X$ given by the composition $\delta_{X} \circ\left(\Gamma_{1} \times \Gamma_{2}\right)$, then $\Gamma_{1, *}\left(\alpha_{1}\right) \cdot \Gamma_{2, *}\left(\alpha_{2}\right)=\Gamma_{*}\left(\alpha_{1} \times \alpha_{2}\right)$, here $\times$ is the exterior product. Now on one hand, the Albanese invariant of the cycle $\alpha_{1} \times \alpha_{2} \in \mathrm{CH}_{0}\left(C_{1} \times C_{2}\right)$ alg is trivial. On the other hand, by the universal property of algebraic representative, whose existence is proved in $(v)$, we have the commutative diagram

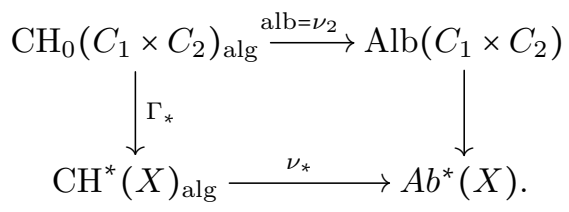

Hence $\Gamma_{*}\left(\alpha_{1} \times \alpha_{2}\right)$ belongs to $\operatorname{ker}\left(\nu_{*}\right)$, which is a finite abelian group by $(v)$. In other words, the image of the intersection product $\mathrm{CH}^{*}(X)_{\mathrm{alg}} \otimes \mathrm{CH}^{*}(X)_{\mathrm{alg}} \rightarrow \mathrm{CH}^{*}(X)_{\text {alg }}$ is annihilated by some integer. However, this image is also divisible, hence must be zero.

Remark 2.17 (Beauville's conjectures on supersingular abelian varieties). Let $A$ be an abelian variety of dimension $g$. Recall Beauville's decomposition [12] on rational Chow groups: for any $0 \leq i \leq g$,

$$
\mathrm{CH}^{i}(A)_{\mathbb{Q}}=\bigoplus_{s=i-g}^{i} \mathrm{CH}^{i}(A)_{(s)},
$$

where $\mathrm{CH}^{i}(A)_{(s)}$ is the common eigen-space of the multiplication-by- $m$ map (with eigenvalue $m^{2 i-s}$ ) for all $m \in \mathbb{Z}$. As is pointed out in [27], when $A$ is supersingular, Corollary 2.16 confirms all the conjectures proposed in [10] and [12]. A remarkable feature of the Chow rings of supersingular abelian varieties that is not shared by other varieties with supersingular abelian motives in general is that the decomposition $\mathrm{CH}^{*}(X)_{\mathbb{Q}}=\mathrm{CH}^{*}(X)_{(0)} \oplus \mathrm{CH}^{*}(X)_{(1)}$ is multiplicative, that is, in addition to the properties $(i)-(v i)$ in Corollary 2.16, we have that the subspace $\mathrm{CH}^{*}(X)_{(0)}$ is closed under the intersection product. We will see in $\S 3.5$ how this supplementary feature is extended to another class of supersingular varieties, namely the supersingular irreducible symplectic varieties.

Remark 2.18. There is more precise information on the Griffiths group of codimension two cycles on a supersingular abelian variety if the base field is the algebraic closure of a finite field of characteristic $p$ : in [33], Gordon-Joshi showed that it is at most a $p$-primary torsion group.

\section{SUPERSINGULAR IRREDUCIBLE SYMPLECTIC VARIETIES}

We now start to investigate the various notions of supersingularity introduced in the previous section, as well as their relations, for a special class of varieties, namely 
the irreducible symplectic varieties. As in the case for K3 surfaces, we expect that all these notions are equivalent in this case.

\subsection{Irreducible symplectic varieties.}

Definition 3.1. Let $X$ be a connected smooth projective variety defined over $k$ of characteristic $p>2$, and let $\Omega_{X / k}^{2}$ be the locally free sheaf of algebraic 2-forms over $k$. $X$ is called irreducible symplectic if

(1) $\pi_{1}^{e ́ t}(X)=0$;

(2) the Frölicher spectral sequence $E_{1}^{i, j}=H^{j}\left(X, \Omega_{X / k}^{i}\right) \Rightarrow H_{\mathrm{dR}}^{i+j}(X / k)$ degenerates at $E_{1}$

(3) $H^{0}\left(X, \Omega_{X / k}^{2}\right)$ is spanned by a nowhere degenerate closed algebraic 2-form;

In particular, $X$ is even-dimensional with trivial canonical bundle.

Remark 3.2. The conditions (1) and (2) in Definition 3.1 together imply that

$$
H^{1}\left(X, \mathcal{O}_{X}\right)=H^{0}\left(X, \Omega_{X}^{1}\right)=H^{0}\left(X, T_{X}\right)=0 .
$$

Due to the lack of Hodge symmetry in positive characteristic, we do not know whether $H^{2}\left(X, \mathcal{O}_{X}\right) \simeq k$, although we expect it is the case.

Example 3.3. The construction methods for irreducible symplectic varieties over fields of positive characteristic are somehow limited as is in the case over the complex numbers. Let us mention some examples which will be studied later in this paper:

(i) the 2-dimensional irreducible symplectic varieties are K3 surfaces;

(ii) the Hilbert scheme of length- $n$ subschemes on a K3 surface ([11]);

(iii) smooth moduli spaces of stable sheaves (or more generally stable complexes with respect to some Bridgeland stability condition) on a K3 surface ([62], [68], [39], [7], [8]), under some mild numeric conditions on the Mukai vector and the polarization (see Proposition 4.5);

(iv) the generalized Kummer varieties $K_{n}(A)$ associated to an abelian surface $A$ ([11]), provided that it is smooth over $k$ (the smoothness condition does not always hold, see [79]). By definition, it is the fiber of the isotrivial fibration $s: A^{[n+1]} \rightarrow A$, where $s$ sends a subscheme to the summation of its support (with multiplicities).

$(v)$ The Albanese fiber of a smooth moduli space of stable sheaves (or more generally Bridgeland stable objects in the derived category) on an abelian surface ([62], [91]), under some mild numeric conditions on the Mukai vector and the polarization, provided that it is smooth over $k$ (see Proposition 7.1).

(vi) the Fano varieties of lines of smooth cubic fourfolds ([14]).

(vii) O'Grady provides in [69] and [70] other examples by symplectic resolutions of some singular moduli space of sheaves on K3 and abelian surfaces.

We say that an irreducible symplectic variety is of $K 3^{[n]}$-type if it is deformation equivalent to example (ii) above; and of generalized Kummer type if it is deformation equivalent to example (iv) above. The examples (iii) and (vi) are of $K 3^{[n]}$-type ; the example $(v)$ is of generalized Kummer type; while examples in (vii) are of different deformation types from the previous ones.

3.2. Artin supersingularity and formal Brauer group. As in the situation over the complex numbers, we expect that the second cohomology of an irreducible symplectic variety should control most of its geometry, up to birational equivalence. This motivates us to single out the following most important piece in Definition 2.2: 
Definition 3.4. Let $X$ be an irreducible symplectic variety of dimension $2 n$ over $k$. $X$ is called $2^{\text {nd }}$-Artin supersingular if the $F$-crystal $\left(H^{2}(X), \varphi\right)$ is supersingular, i.e. the Newton polygon $\mathrm{Nt}^{2}(X)$ is a straight line (of slope 1 ).

For a K3 surface, Artin defined its supersingularity originally in [5] by looking at its formal Brauer group $\widehat{\mathrm{Br}}$, which turns out to be equivalent to the supersingularity of the $F$-crystal $\left(H^{2}(X), \varphi\right)$ discussed before. More generally, Artin and Mazur made the observation in [6] that the formal Brauer group $\widehat{\mathrm{Br}}$ actually fits into a whole series of formal groups. Recall that a formal group is simply a group object in the category of formal schemes, we refer to [56] for the general theory of (commutative) formal group. For any $i \in \mathbb{N}$, consider the following functor :

$$
\begin{aligned}
\Phi_{X}^{i}:(\text { Artin local } k \text {-algebras }) & \rightarrow \text { (Abelian groups) } \\
R & \mapsto \operatorname{ker}\left(H_{\text {ét }}^{i}\left(X \times_{k} R, \mathbb{G}_{m}\right) \rightarrow H_{\text {êt }}^{i}\left(X, \mathbb{G}_{m}\right)\right),
\end{aligned}
$$

If $H^{i-1}\left(X, \mathcal{O}_{X}\right)=0$, this functor is pro-representable by a formal group $\widehat{\Phi}^{i}(X)$, called the $i$-th Artin-Mazur formal group of $X$. In particular, $\widehat{\Phi}^{1}(X)=\widehat{\operatorname{Pic}}(X)$ is the formal Picard group and $\widehat{\Phi}^{2}(X)=\widehat{\operatorname{Br}}(X)$ is the formal Brauer group of $X$. Moreover, if $H^{i+1}\left(X, \mathcal{O}_{X}\right)$ vanishes, then the functor $\Phi_{X}^{i}$, as well as $\widehat{\Phi}^{i}(X)$, is formally smooth with abelian Lie algebra $H^{i}\left(X, \mathcal{O}_{X}\right)$.

In particular, for an irreducible symplectic variety $X$, we always have the vanishing of $H^{1}\left(X, \mathcal{O}_{X}\right)$, which implies that the formal Brauer group $\widehat{\operatorname{Br}}(X)$ is a welldefined formal group, i.e. the functor $\widehat{\Phi}^{2}$ is pro-representable. As an analogue of Artin's notion for the supersingularity of K3 surfaces in [5], we make the following:

Definition 3.5. An irreducible symplectic variety $X$ is called Artin $\widehat{\mathrm{Br}}$-supersingular if $\widehat{\operatorname{Br}}(X)$ is isomorphic to the formal additive group $\widehat{\mathbb{G}}_{a}$.

Remark 3.6. Let $X$ be an irreducible symplectic variety defined over $k$.

- $\widehat{\operatorname{Br}}(X)$ is expected to be formally smooth. We know that it is the case when $H^{3}\left(X, \mathcal{O}_{X}\right)$ vanishes (for instance, for $K 3^{[n]}$-type varieties).

- Provided that $\operatorname{dim} H^{2}\left(X, \mathcal{O}_{X}\right)=1$ and $\widehat{\operatorname{Br}}(X)$ is formally smooth (as is for most known examples of irreducible symplectic varieties of $K 3^{[n]}$-type), $\widehat{\operatorname{Br}}(X)$ is a smooth one-dimensional formal group and it is uniquely determined by its height ([56], [6]). In this case, $\widehat{\operatorname{Br}}(X)$ is isomorphic to $\widehat{\mathbb{G}}_{a}$ if and only if the height is $\infty$.

Recall that the Cartier ring Cart $(k)$ is by definition the quotient of the ring of non-commutative power series in $V$ and polynomials in $F$ with coefficients in the Witt ring $W(k)$, modulo the following relations for all $r \in W(k)$,

$$
F V=p ; V r F=V(r) ; F r=\sigma(r) F ; r V=V \sigma(r),
$$

where $V$ and $\sigma$ on the right hand sides are Verschiebung and Frobenius of $W(k)$. Note that a left Cart $(k)$-module which is torsion-free of finite rank over $W(k)$ is naturally an $F$-crystal with $\varphi$ given by the multiplication by $F$. The DieudonnéCartier module of the formal group $\widehat{\Phi}^{i}(X)$, which is a left module over $\operatorname{Cart}(k)$, provides a link to the $i$-th crystalline cohomology of $X$.

Proposition 3.7. ([6, Corollary 2.7]) Let $X$ be a smooth projective variety of $k$. Let $D\left(\widehat{\Phi}^{i}(X)\right)$ be the Dieudonné-Cartier module of $\widehat{\Phi}^{i}(X)$. If $\widehat{\Phi}^{i}(X)$ is formally 
smooth, then there is an isomorphism of F-isocrystals:

$$
D\left(\Phi^{i}(X)\right) \otimes K \cong\left(H^{i}(X)_{K}\right)_{[0,1[},
$$

where the right hand side denotes the direct sum of the sub-F-isocrystals of $H^{i}(X)_{K}$ with slopes strictly less than 1.

Using this result, one can obtain the following consequence which guarantees that the two notions of supersingularity in Definitions 3.4 and 3.5 coincide under mild condition, which presumably always holds for irreducible symplectic varieties.

Proposition 3.8. Let $X$ be an irreducible symplectic variety. If the functor $\Phi_{X}^{2}$ is formally smooth and $H^{2}\left(X, \mathcal{O}_{X}\right) \simeq k$, then $X$ is Artin $\widehat{\mathrm{Br}}$-supersingular if and only if the F-crystal $\left(H^{2}(X), \varphi\right)$ is supersingular, that is, $X$ is $2^{\text {nd }}$-Artin supersingular.

Proof. Under the hypothesis, $\widehat{\operatorname{Br}}(X)$ is a 1-dimensional formal Lie group, hence is classified by its height. It is the formal additive group if and only if $D\left(\Phi^{2}(X)\right)_{K}$ is zero. Then we can conclude by Proposition 3.7, since the Newton polygon of $\left(H^{2}(X), \varphi\right)$ is a straight line if and only if there is no sub- $F$-isocrystals with slopes strictly less than 1 .

Furthermore, similar to the case of K3 surfaces [5, Theorem 1.1], the Picard number behaves very well for families of Artin $\widehat{B r}$-supersingular irreducible symplectic varieties :

Corollary 3.9. Let $\pi: \mathfrak{X} \rightarrow B$ be a smooth projective family of Artin $\widehat{B r}$-supersingular irreducible symplectic varieties over a connected base scheme B. Assume either $\operatorname{Pic}^{\tau}(\mathfrak{X} / B)$ is smooth or the cohomology groups of $\mathfrak{X} / B$ are torsion free. Write $\mathfrak{X}_{b}=\pi^{-1}(b)$ for $b \in B$, then the Picard number $\rho\left(\mathfrak{X}_{b}\right)$ is constant for all $b \in B$. In particular, all the fibers of $\pi$ are $2^{\text {nd }}$-Shioda supersingular if and only if one of them is $2^{\text {nd }}$-Shioda supersingular.

Proof. The proof is similar to [5, Theorem 1.1]. It suffices to show $\rho\left(\mathfrak{X}_{\eta}\right)=\rho\left(\mathfrak{X}_{0}\right)$ for any $B=\operatorname{Spec} k[[t]]$, where $\mathfrak{X}_{\eta}$ is the generic fiber and $\mathfrak{X}_{0}$ is the special fiber. The supersingular assumption indicates that the $F$-crystal $H^{2}\left(\mathfrak{X}_{b} / W\right)$ is constant. Then the result in $[5,61,22]$ imply that the cokernel of the specialization map

$$
\mathrm{NS}\left(\mathfrak{X}_{\eta}\right) \rightarrow \operatorname{NS}\left(\mathfrak{X}_{0}\right)
$$

is finite and annihilated by powers of $p$. It follows that $\rho\left(\mathfrak{X}_{\eta}\right)=\rho\left(\mathfrak{X}_{0}\right)$.

3.3. Artin supersingularity vs. Shioda supersingularity. As is mentioned in Remark 2.5, the $2^{\text {nd }}$-Artin supersingularity is a priori weaker than the $2^{\text {nd }}$-Shioda supersingularity. In the other direction, for irreducible symplectic varieties, we have the Tate conjecture for certain irreducible symplectic varieties.

Theorem 3.10 (Charles [18]). Let $Y$ be an irreducible symplectic variety variety of dimension $2 n$ over $\mathbb{C}$ and let $L$ be an ample line bundle on $Y$ and $d=c_{1}(L)^{2 n}$. Assume that $p$ is prime to $d$ and that $p>2 n$. Suppose that $Y$ can be defined over a finite unramified extension of $\mathbb{Q}_{p}$ and that $Y$ has good reduction at $p$. If the Beauville-Bogomolov form of $Y$ induces a non-degenerate quadratic form on the reduction modulo $p$ of the primitive lattice in the second cohomology group of $Y$, then the reduction of $Y$ at $p$, denoted by $X$, satisfies the Tate conjecture for divisors.

In particular, when $Y$ is an irreducible symplectic variety of $K 3$ type and $p>$ $2 n, p+d$, then $X$ satisfies the Tate conjecture for divisors. 
This yields the following consequence:

Corollary 3.11. Suppose $X$ is an irreducible symplectic variety defined over $k$ satisfying all the conditions in Theorem 3.10, then $X$ is $2^{\text {nd }}$-Artin supersingular if and only if $X$ is $2^{\text {nd }}$-Shioda supersingular.

Example 3.12. If $X$ is the Fano variety of lines on a smooth cubic fourfold and $p \geq 5$, then $X$ is $2^{n d}$-Artin supersingular if and only if it is $2^{n d}$-Shioda supersingular.

A more difficult question is to go beyond the second cohomology to ask whether $X$ is fully Shioda supersingular (Definition 2.4) and hence fully Artin supersingular (Definition 2.2) if $X$ is $2^{\text {nd }}$-Artin supersingular. We will prove this for smooth moduli spaces of stable sheaves on K3 surfaces and abelian surfaces (Corollaries 1.4 and 1.6). We conjecture that this should hold in general:

Conjecture 3.13 (Equivalence Conjecture). Let $X$ be an irreducible symplectic variety defined over an algebraically closed field of positive characteristic. Then the following conditions are equivalent:

- $X$ is $2^{\text {nd }}$-Artin supersingular.

- $X$ is $2^{\text {nd }}$-Shioda supersingular.

- $X$ is fully Artin supersingular.

- $X$ is fully Shioda supersingular.

There are some easy implications in this conjecture, see Remark 2.5:

Fully Shioda supersingular $\Longrightarrow$ Fully Artin supersingular

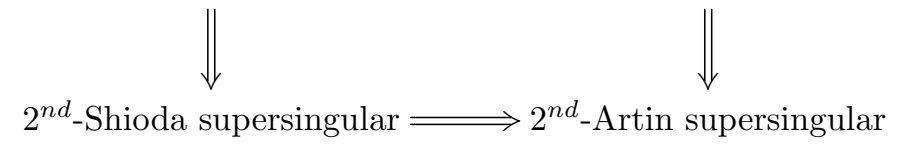

which is part of the diagram in Figure 1 in the introduction.

Conjecture 3.13 can be viewed as a strengthening of the Tate conjecture for supersingular irreducible symplectic varieties. Apart from the principle that the second cohomology should control the whole cohomology for such varieties, the more fundamental reason to believe it comes from the motivic consideration (Conjecture $3.16)$, which will be explained in $\S 3.5$.

3.4. Unirationality $v s$. supersingularity. In the direction of looking for a geometric characterization of the supersingularity for irreducible symplectic varieties, we expect the following conjecture. The motivation comes from the unirationality Conjecture 1.1 for K3 surfaces, which is solved by Rudakov-Šafarevič [77] in characteristic 2, Liedtke [53] in characteristic at least 5 and Bragg-Lieblich [16] in general.

Conjecture 3.14 (Unirationality Conjecture). Let $X$ be a smooth projective supersingular irreducible symplectic variety. Then $X$ is $2^{\text {nd }}$-Artin supersingular if and only if $X$ is unirational.

A slightly weaker version is the following. Recall that a smooth projective variety is rationally chain connected if any two points can be connected by a chain of rational curves.

Conjecture 3.15 (RCC Conjecture). With the same assumption as above, $X$ is $2^{\text {nd }}$-Artin supersingular if and only if $X$ is rationally chain connected. 
As evidence, we will verify the unirationality conjecture for most smooth moduli spaces of stable sheaves on K3 surfaces (Corollary 1.4) and prove the RCC conjecture for most smooth Albanese fibers of moduli spaces of stable sheaves on abelian surfaces (Corollary 1.6). However, the unirationality conjecture remains open even for generalized Kummer varieties of dimension at least four. It is also very interesting to investigate these conjectures for irreducible symplectic varieties of O'Grady-6 and O'Grady-10 types.

Thanks to Theorem 2.8, some implications are easy in the previous conjectures, namely:

Unirational $\Longrightarrow \mathrm{RCC} \Longrightarrow 2^{\text {nd }}$-Shioda supersingular $\Longrightarrow 2^{\text {nd }}$-Artin supersingular

This is part of the diagram in Figure 1 in the introduction.

3.5. Algebraic cycles and supersingularity. Over the field of complex numbers, thanks to the Kuga-Satake construction ( $c f$. [46]), one expects that the Chow motive of any projective irreducible symplectic variety is of abelian type. In positive characteristic, it is also natural to conjecture that any supersingular irreducible symplectic variety has supersingular abelian Chow motive, in the sense of Definition 2.14 .

Conjecture 3.16 (Abelian Motive Conjecture). Let $X$ be a $2^{\text {nd }}$-Artin supersingular irreducible symplectic variety. Then the rational Chow motive of $X$ belongs to $\mathcal{M}^{s s a b}$, the idempotent-complete additive tensor subcategory of $\mathrm{CHM}_{\mathbb{Q}}$ generated by supersingular abelian varieties.

Thanks to Theorem 2.13 and Corollary 2.16, the Abelian Motive Conjecture 3.16 implies the Equivalence Conjecture (i.e. full Shioda supersingularity) and Supersingular Bloch-Beilinson Conjecture in the introduction:

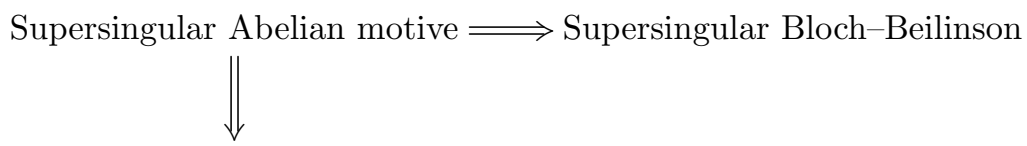

Fully Shioda supersingular

which is part of the diagrams in Figure 1 and Figure 2.

Summarize differently: for any fully supersingular variety having supersingular abelian motive, there is a short exact sequence of graded vector spaces

$$
0 \rightarrow A b^{*}(X)_{\mathbb{Q}} \rightarrow \mathrm{CH}^{*}(X)_{\mathbb{Q}} \rightarrow \overline{\mathrm{CH}}^{*}(X)_{\mathbb{Q}} \rightarrow 0,
$$

where $\overline{\mathrm{CH}}^{*}:=\mathrm{CH}^{*} / \equiv$ and $A b^{*}(X)_{\mathbb{Q}}=\mathrm{CH}^{*}(X)_{\mathrm{alg}, \mathbb{Q}}=\mathrm{CH}^{*}(X)_{\text {num, } \mathbb{Q}}$. However so far the only thing we can say in general about the ring structure on $\mathrm{CH}^{*}(X)_{\mathbb{Q}}$ given by the intersection product is that $A b^{*}(X)_{\mathbb{Q}}$ forms a square zero graded ideal. It is the insight of Beauville [13] that reveals a supplementary structure on $\mathrm{CH}^{*}(X)_{\mathbb{Q}}$ : if $X$ is moreover irreducible symplectic, then its rational Chow ring should have a multiplicative splitting of the Bloch-Beilinson filtration. In the fully supersingular case, the Bloch-Beilinson filtration is supposed to be (10) and Beauville's splitting conjecture reduces to the supersingular case of the following conjecture.

Conjecture 3.17 (Section Conjecture [30]). Let $X$ be an irreducible symplectic variety over an algebraically closed field. Then the algebra epimorphism $\mathrm{CH}^{*}(X)_{\mathbb{Q}} \rightarrow$ $\overline{\mathrm{CH}}^{*}(X)_{\mathbb{Q}}$ admits a (multiplicative) section whose image contains all Chern classes of the tangent bundle of $X$. 
The study of this conjecture in [30] was inspired by O'Sullvian's theory on symmetrically distinguished cycles on abelian varieties [74], which provides such an algebra section for abelian varieties. This is an important step towards Beauville's general conjecture in [10] and [12]. As a result, we call the elements in the image of the section in Conjecture 3.17 distinguished cycles and denote the corresponding subalgebra by $\mathrm{DCH}^{*}(X)$. By definition, the natural projection $\mathrm{DCH}(X) \rightarrow \overline{\mathrm{CH}}(X)$ is an isomorphism.

Remark 3.18. It is easy to deduce the Supersingular Beauville Splitting Conjecture in the introduction ( $c f$. [13] for the original version in the complex setting) from the other aforementioned conjectures, thus completing Figure $2:$ let $\mathrm{CH}^{*}(X)_{(0)}:=$ $\mathrm{DCH}^{*}(X)$ be the image of the algebra section and $\mathrm{CH}^{*}(X)_{(1)}=\mathrm{CH}^{*}(X)_{\text {alg, } \mathbb{Q}}$ be the algebraically trivial cycles. By the Supersingular Bloch-Beilinson Conjecture, we have $\mathrm{CH}^{*}(X)_{(0)} \oplus \mathrm{CH}^{*}(X)_{(1)}=\mathrm{CH}^{*}(X)_{\mathbb{Q}}$ and $\mathrm{CH}^{*}(X)_{(1)}$ is a square zero ideal. The only remaining multiplicativity condition that $\mathrm{CH}^{*}(X)_{(0)}:=\mathrm{DCH}^{*}(X)$ is closed under the intersection product is the content of the Section Conjecture.

Return to the Section Conjecture 3.17. It is observed in [30] that to establish this conjecture it is both more fundamental and practically easier to verify the Key Condition ( $\star$ ) defined in [30], which goes beyond the scope of irreducible symplectic varieties. Let us give it a brief account in the supersingular situation:

Definition 3.19 (The category $\mathcal{M}_{s d}^{s s a b}$, [30, Definitions 2.1 and 2.3]). The category of symmetrically distinguished supersingular abelian motives, denoted $\mathcal{M}_{s d}^{s s a b}$, is defined as follows :

(i) An object is the following data:

- a positive integer $r \in \mathbb{N}^{*}$;

- a length- $r$ sequence of supersingular abelian varieties $A_{1}, \ldots, A_{r}$;

- a length- $r$ sequence of integers $n_{1}, \ldots, n_{r} \in \mathbb{Z}$;

- an $(r \times r)$-matrix $P:=\left(p_{i, j}\right)_{1 \leq i, j \leq r}$ with $p_{i, j} \in \mathrm{CH}^{\operatorname{dim} A_{i}+n_{j}-n_{i}}\left(A_{i} \times A_{j}\right)_{(0)}$, such that $P \circ P=P$.

Such an object is denoted in the sequel by a triple

$$
\left(A_{1} \sqcup \cdots \sqcup A_{r}, P=\left(p_{i, j}\right),\left(n_{1}, \ldots, n_{r}\right)\right) .
$$

(ii) The group of morphisms (resp. symmetrically distinguished morphisms) from an object $M=\left(A_{1} \sqcup \cdots \sqcup A_{r}, P=\left(p_{i, j}\right),\left(n_{1}, \ldots, n_{r}\right)\right)$ to another object $N=$ $\left(B_{1} \sqcup \cdots \sqcup B_{s}, Q=\left(q_{i, j}\right),\left(m_{1}, \ldots, m_{s}\right)\right)$ is the subgroup of

$$
\bigoplus_{i=1}^{r} \bigoplus_{j=1}^{s} \mathrm{CH}^{\operatorname{dim} A_{i}+m_{j}-n_{i}}\left(A_{i} \times B_{j}\right)
$$

(whose elements are viewed as an $(s \times r)$-matrix) given by

$$
\operatorname{Hom}(M, N):=Q \circ\left(\bigoplus_{i=1}^{r} \bigoplus_{j=1}^{s} \mathrm{CH}^{\operatorname{dim} A_{i}+m_{j}-n_{i}}\left(A_{i} \times B_{j}\right)\right) \circ P
$$

resp.

$$
\operatorname{DHom}(M, N):=Q \circ\left(\bigoplus_{i=1}^{r} \bigoplus_{j=1}^{s} \mathrm{CH}^{\operatorname{dim} A_{i}+m_{j}-n_{i}}\left(A_{i} \times B_{j}\right)_{(0)}\right) \circ P,
$$

where the multiplication law is the one between matrices. The composition is defined as usual by composition of correspondences. 
(iii) The category $\mathcal{M}_{s d}^{s s a b}$ is naturally an additive rigid symmetric monoïdal category.

Be aware that $\mathcal{M}_{s d}^{s s a b}$ is not a subcategoryof $\operatorname{CHM}(k)_{\mathbb{Q}}$ since in the definition of motives, one uses varieties instead of pointed varieties or varieties with additional structures. However, let $\mathcal{M}^{s s a b}$ be the subcategory of $\operatorname{CHM}(k)_{\mathbb{Q}}$ generated by motives of supersingular abelian varieties (Definition 2.14), then there is an equivalence of categories:

$$
F: \mathcal{M}_{s d}^{s s a b} \stackrel{\sim}{\rightarrow} \mathcal{M}^{s s a b},
$$

which send an object $\left(A_{1} \sqcup \cdots \sqcup A_{r}, P=\left(p_{i, j}\right),\left(n_{1}, \ldots, n_{r}\right)\right)$ to the Chow motive $\operatorname{im}\left(P: \oplus_{i=1}^{r} \mathfrak{h}\left(A_{i}\right)\left(n_{i}\right) \rightarrow \oplus_{i=1}^{r} \mathfrak{h}\left(A_{i}\right)\left(n_{i}\right)\right)$.

For any object $M$ in $\mathcal{M}_{s d}^{\text {ssab }}$ and any $i \in \mathbb{Z}$, the $i$-th Chow group $\mathrm{CH}^{i}(M)$ is defined to be $\mathrm{CH}^{i}(F(M))_{\mathbb{Q}}$, that is, $\operatorname{Hom}_{\mathcal{M}_{s d}^{s s a b}}(\mathbb{1}(-i), M)$. It has the subspace of symmetrically distinguished cycles

$$
\operatorname{DCH}^{i}(M):=\operatorname{DHom}_{\mathcal{M}_{s d}^{s s a b}}(\mathbb{1}(-i), M) .
$$

Definition 3.20 (Markings and distinguished cycles). Let $X$ be a smooth projective variety with supersingular abelian Chow motive: $\mathfrak{h}(X) \in \mathcal{M}^{\text {ssab }}$. A marking for $X$ consists of an object $M \in \mathcal{M}_{s d}^{s s a b}$ together with an isomorphism

$$
\phi: \mathfrak{h}(X) \stackrel{\simeq}{\rightarrow} F(M) \quad \text { in } \operatorname{CHM}(k)_{\mathbb{Q}},
$$

where $F: \mathcal{M}_{s d}^{s s a b} \stackrel{\simeq}{\rightarrow} \mathcal{M}^{s s a b}$ is the equivalence in Definition 3.19 .

An algebraic cycle $z \in \mathrm{CH}(X)_{\mathbb{Q}}$ is called distinguished, if its image via the induced isomorphism $\phi_{*}: \mathrm{CH}(X)_{\mathbb{Q}} \stackrel{\sim}{\rightarrow} \mathrm{CH}(M)$ belongs to $\mathrm{DCH}(M)$. The subgroup of distinguished cycles, which depends on the marking $\phi$, is denoted by $\mathrm{DCH}_{\phi}(X)$.

By construction and Corollary 2.16 (iii), (iv), the composition

$$
\mathrm{DCH}_{\phi}^{i}(X) \rightarrow \mathrm{CH}^{i}(X)_{\mathbb{Q}} \rightarrow \overline{\mathrm{CH}}^{i}(X)_{\mathbb{Q}}
$$

is an isomorphism ( $c f$. [30, Lemma 3.3]). In other words, $\phi$ provides a section (as graded vector spaces) of the natural projection $\mathrm{CH}(X)_{\mathbb{Q}} \rightarrow \overline{\mathrm{CH}}(X)_{\mathbb{Q}}$.

The natural question is whether $\operatorname{DCH}_{\phi}(X)$ provides the desired multiplicative section. This led us to the following key condition introduced in [30, §3.2].

Definition 3.21. For a smooth projective variety $X$ with $\mathfrak{h}(X) \in \mathcal{M}^{s s a b}$, we say a marking $\phi: \mathfrak{h}(X) \stackrel{\simeq}{\rightarrow} F(M)$ (with $M \in \mathcal{M}_{s d}^{s s a b}$ ) satisfies $(\star)$, if

$\left(\star_{\text {Mult }}\right)$ (Multiplicativity) the small diagonal $\delta_{X}$ belongs to $\operatorname{DCH}_{\phi^{\otimes 3}}\left(X^{3}\right)$, that is, under the induced isomorphism $\phi_{*}^{\otimes 3}: \mathrm{CH}\left(X^{3}\right) \stackrel{\simeq}{\rightarrow} \mathrm{CH}\left(M^{\otimes 3}\right)$, the image of the small diagonal $\delta_{X}$ is in $\operatorname{DCH}\left(M^{\otimes 3}\right)$;

$\left({ }^{\star}\right.$ Chern) (Chern classes) all Chern classes of $T_{X}$ belong to $\operatorname{DCH}_{\phi}(X)$.

We say that $X$ satisfies $(\star)$ if such a marking exists.

The following proposition is proved in [30, Proposition 3.12].

Proposition 3.22. Let $X$ be a smooth projective variety with abelian motive. If $X$ satisfies $(\star)$, then there exists a section, as graded algebras, for the natural epimorphism $\mathrm{CH}(X)_{\mathbb{Q}} \rightarrow \overline{\mathrm{CH}}(X)_{\mathbb{Q}}$ and all Chern classes of $X$ are in the image of this section.

In other words, under $(\star)$, we have a graded $\mathbb{Q}$-subalgebra $\mathrm{DCH}(X)$ of the Chow ring 
$\mathrm{CH}(X)_{\mathbb{Q}}$, which contains all the Chern classes of $X$ and is mapped isomorphically to $\overline{\mathrm{CH}}(X)_{\mathbb{Q}}$. We call elements of $\mathrm{DCH}(X)$ distinguished cycles of $X$.

Therefore, to show the Section Conjecture 3.17 for supersingular irreducible symplectic varieties, it suffices to present a marking (Definition 3.20) satisfying the key condition ( $\star$ ) in Definition 3.21. We will indeed construct such markings, hence prove the Section Conjecture, for two series of examples of supersingular irreducible symplectic varieties, namely, smooth moduli spaces of stable sheaves on a supersingular K3 surface and smooth Albanese fibers of smooth moduli spaces of stable sheaves on a supersingular abelian surface.

3.6. Birational irreducible symplectic varieties. In this subsection, we compare the Chow rings and cohomology rings of two birationally equivalent irreducible symplectic varieties.

The starting point is the following result of Rieß, built on Huybrechts' fundamental work [39]. Actually her proof yields the following more precise result. We denote by $\Delta_{X} \subset X \times X$ the diagonal and $\delta_{X}=\{(x, x, x) \mid x \in X\} \subset X \times X \times X$ the small diagonal for a variety $X$.

Theorem 3.23 (Rieß $[76, \S 3.3$ and Lemma 4.4]). Let $X$ and $Y$ be d-dimensional projective irreducible symplectic varieties over $k=\mathbb{C}$. If they are birational, then there exists a correspondence $Z \in \mathrm{CH}_{d}(X \times Y)$ such that

(i) $(Z \times Z)_{*}: \mathrm{CH}_{d}(X \times X) \rightarrow \mathrm{CH}_{d}(Y \times Y)$ sends $\Delta_{X}$ to $\Delta_{Y}$;

(ii) $(Z \times Z \times Z)_{*}: \mathrm{CH}_{d}(X \times X \times X) \rightarrow \mathrm{CH}_{d}(Y \times Y \times Y)$ sends $\delta_{X}$ to $\delta_{Y}$.

(iii) $Z_{*}: \mathrm{CH}(X) \rightarrow \mathrm{CH}(Y)$ sends $c_{i}(X)$ to $c_{i}(Y)$ for any $i \in \mathbb{N}$;

(iv) $Z$ induces an isomorphism of algebra objects $\mathfrak{h}(X) \rightarrow \mathfrak{h}(Y)$ in $\operatorname{CHM}(k)$ with inverse given by ${ }^{t} Z$.

In particular, $Z$ induces an isomorphism between their Chow rings and cohomology rings.

Note that $(i v)$ is a reformulation of $(i)$ and $(i i)$. Our objective is to extend Rieß's result to other algebraically closed fields under the condition of liftability. More precisely,

Proposition 3.24. Let $X$ and $Y$ be two birationally equivalent projective irreducible symplectic varieties defined over an algebraically closed field $k$. If the characteristic of $k$ is positive, we assume moreover that $X$ and $Y$ are both liftable to $\mathcal{X}$ and $\mathcal{Y}$ over a characteristic zero base $W$ with generic fibers $\mathcal{X}_{\eta_{W}}$ and $\mathcal{Y}_{\eta_{W}}$ being geometrically birational. Then the same results as in Theorem 3.23 hold.

Proof. We first treat the case where $\operatorname{char}(k)=0$. Without loss of generality, we can assume that $k$ is finitely generated over its prime field $\mathbb{Q}$, and fix an embedding $k \rightarrow \mathbb{C}$. As $X_{\mathbb{C}}$ and $Y_{\mathbb{C}}$ are birational complex irreducible symplectic varieties, we have a cycle $Z_{\mathbb{C}} \in \mathrm{CH}^{d}\left(X_{\mathbb{C}} \times_{\mathbb{C}} Y_{\mathbb{C}}\right)$ verifying the properties in Theorem 3.23. Let $L$ be a finitely generated field extension of $k$, such that $Z_{\mathbb{C}}$, as well as all the rational equivalences involved, is defined over $L$. Take a smooth connected $k$-variety $B$ whose function field $k(B)=\kappa\left(\eta_{B}\right)=L$ and choose a closed point $b \in B(k)$. Let $Z \in \mathrm{CH}^{d}\left(X_{\eta_{B}} \times_{L} Y_{\eta_{B}}\right)$ with $Z \otimes_{L} \mathbb{C}=Z_{\mathbb{C}}$ and such that $Z$ satisfies the properties in Theorem 3.23 for $X_{\eta_{B}}$ and $Y_{\eta_{B}}$. Now the specialization of $Z$ from the generic point $\eta_{B}$ to the closed point $b$ gives rise to a cycle $\operatorname{sp}(Z) \in \mathrm{CH}^{d}(X \times Y)$, which satisfies 
all the properties of Theorem 3.23 because specialization respects compositions of correspondences, diagonals and small diagonals ( $c f .[31, \S 20.3])$.

In the case where $\operatorname{char}(k)>0$, let $W, \mathcal{X}$ and $\mathcal{Y}$ be as in the statement. Denote by $K=\operatorname{Frac}(W), w$ the closed point of $W$ with residual field $k$. By hypothesis, $\mathcal{X}_{K}$ and $\mathcal{Y}_{K}$ are geometrically birational, hence the result in characteristic zero proved in the previous paragraph implies that there exist a finite field extension $L / K$, an algebraic cycle $Z \in \mathrm{CH}^{d}\left(\mathcal{X}_{L} \times_{L} \mathcal{Y}_{L}\right)$ which satisfies all the properties of Theorem 3.23 for $\mathcal{X}_{L}$ and $\mathcal{Y}_{L}$. Take any $W$-scheme $B$ with $\kappa\left(\eta_{B}\right)=L$ and choose a closed point $b$ of $B$ in the fiber of $w$, then $\kappa(b)=k$ since $k$ is algebraically closed. Then as before, the specialization of $Z$ from the generic point $\eta_{B}$ to the closed point $b$ yields a cycle $\operatorname{sp}(Z) \in \mathrm{CH}^{d}\left(X \times_{k} Y\right)$ which inherits all the desired properties from $Z$.

In view of Proposition 3.24, the following notions are convenient.

Definition 3.25 ((Quasi-)liftably birational equivalence). Two irreducible symplectic varieties $X$ and $Y$ are called liftably birational if they are both liftable over some base $W$ of characteristic zero with geometric generic fibers being birationally equivalent.

Two irreducible symplectic varieties $X$ and $Y$ are called quasi-liftably birational if there exists a (finite) sequence of irreducible symplectic varieties

$$
X=X_{0}, X_{1}, \cdots, X_{m}=Y,
$$

such that for any $0 \leq i \leq m-1, X_{i}$ and $X_{i+1}$ are liftably birational.

As a consequence of Proposition 3.24, the Chow rings and the cohomology rings of irreducible symplectic varieties are invariant under quasi-liftably birational equivalences.

Remark 3.26. It is expected that any two birational irreducible symplectic varieties over algebraically closed fields always have isomorphic Chow motivic algebras (hence isomorphic Chow rings, cohomology rings etc.) without the hypothesis of liftability. However, we think a more thorough study of the deformation theory for irreducible symplectic varieties over fields of positive characteristic is necessary in order to establish results in this direction.

\section{Moduli spaces of Stable Sheaves on K3 Surfaces}

4.1. Preliminaries on K3 surfaces over positive characteristic. Some useful facts needed later on K3 surfaces are collected here. We start with the NéronSeveri lattices of supersingular K3 surfaces. Let $S$ be a smooth projective K3 surface defined over $k$, an algebraically closed field of positive characteristic $p$. As the Tate conjecture holds for K3 surfaces over finite fields of any characteristic ([18], [55], [19], [43]), $S$ is Artin supersingular if and only if it is Shioda supersingular (cf. the argument in [54, Theorem 4.8]), hence the notion of supersingularity has no ambiguity for K3 surfaces.

By Artin's work [5], if $S$ is supersingular, the discriminant of the intersection form on $\operatorname{NS}(S)$ is $\operatorname{disc} \operatorname{NS}(S)= \pm p^{2 \sigma(S)}$ for some integer $1 \leq \sigma(S) \leq 10$, which is called the Artin invariant of $S$. The lattice $\mathrm{NS}(S)$ is uniquely determined, up to isomorphism, by its Artin invariant $\sigma(S)$. Such lattices are completely classified by Rudakov-Šaferevič in $[77, \S 2]$ and when $p>2$, there is a refinement due to Shimada [80]. 
We summarize their results as follows. Given a lattice $\Lambda$ and an integer $n$, we denote by $\Lambda(n)$ the lattice obtained by multiplying its bilinear form by $n$.

Proposition 4.1 (Supersingular K3 lattices). Let $S$ be a supersingular K3 surface defined over an algebraically closed field of positive characteristic $p$. The lattice $\operatorname{NS}(S)$ is isomorphic to $-\Lambda_{\sigma(S)}$. When $p>2$, the intersection form of $\Lambda_{\sigma(S)}$ is given as below

(i) $\sigma(S)<10, \Lambda_{\sigma(S)}= \begin{cases}U \oplus V_{20,2 \sigma(S)}^{(p)}, & \text { if } p \equiv 3 \bmod 4, \text { and } 2+\sigma(S) \\ U \oplus H^{(p)} \oplus V_{16,2 \sigma(S)}^{(p)}, & \text { otherwise }\end{cases}$

(ii) $\sigma(S)=10, \Lambda_{10}=U(p) \oplus H^{(p)} \oplus V_{16,16}^{(p)}$,

Here, $U$ is the hyperbolic plane,

$$
H^{(p)}=\left(\begin{array}{cccc}
2 & 1 & 0 & 0 \\
1 & (q+1) / 2 & 0 & \gamma \\
0 & 0 & p(q+1) / 2 & p \\
0 & \gamma & p & 2\left(p+\gamma^{2}\right) / q
\end{array}\right)
$$

satisfying that the prime $q \equiv 3 \bmod 8,\left(\frac{-q}{p}\right)=-1, \gamma^{2}+p \equiv 0 \bmod q$, and

$$
V_{m, n}^{(p)}=V_{0} \cup\left(\frac{1}{2} \sum_{1}^{m} e_{i}+V_{0}\right)
$$

where

$$
V_{0}=\left\langle\sum_{i=1}^{m} a_{i} e_{i} \mid \sum a_{i} \equiv 0 \bmod 2\right\rangle \subseteq \oplus \mathbb{Z} e_{i} ; e_{i} e_{j}= \begin{cases}0, & \text { if } i \neq j, \\ 1, & \text { if } i=j>n, \\ p, & \text { otherwise. }\end{cases}
$$

When $p=2, \Lambda_{\sigma(S)}$ has been explicitly classified in [77, §2, P.157], we will only use the fact that it contains the hyperbolic lattice $U$ as a direct summand when $\sigma(S)$ is odd and $U(2)$ when $\sigma(S)$ is even. As a consequence, Liedtke [53] shows the existence of nice elliptic fibrations on supersingular K3 surfaces.

Corollary 4.2 (cf. [53, Proposition 3.9]). Let $S$ a supersingular K3 surface defined over an algebraically closed field of positive characteristic $p>2$. Then $S$ admits an elliptic fibration with a section if $\sigma(S) \leq 9$ and an elliptic fibration with a multisection of degree $p$ when $\sigma(S)=10$.

We will need elliptic fibrations with more special properties on supersingular K3 surfaces. A strengthening of Corollary 4.2 will be given in Theorem 4.15.

Let us turn to the liftability problem of K3 surfaces. Ogus [71, Corollary 2.3] (attributed also to Deligne) shows that every polarized K3 surface admits a projective lift. A more useful result is the following.

Proposition 4.3 (Lifting K3 surfaces with line bundles [52, Appendix A.1 (iii)], [19, Proposition 1.5]). Let $S$ be a smooth K3 surface over an algebraically closed field $k$ of characteristic $p>0$. Let $\Sigma \subseteq \mathrm{NS}(S)$ be a saturated subgroup of rank $<11$ containing an ample divisor. Then there exist a complete discrete valuation ring $W^{\prime}$ of characteristic 0 with fraction field $K^{\prime}$ and residue field $\kappa$ containing $k, a$ relative $K 3$ surface

$$
\mathcal{S} \rightarrow \operatorname{Spec}\left(W^{\prime}\right)
$$


with $\mathcal{S}_{\kappa} \cong S \times_{k} \kappa$, such that the specialization map gives an isomorphism

$$
\mathrm{NS}\left(\mathcal{S}_{\overline{K^{\prime}}}\right) \rightarrow \Sigma \text {. }
$$

where $\mathcal{S}_{\overline{K^{\prime}}}$ is the geometric generic fiber over the algebraic closure $\overline{K^{\prime}}$.

4.2. Moduli spaces of stable sheaves. One important source of examples of $K 3^{[n]}$-type irreducible symplectic varieties is the moduli spaces of stable sheaves on a K3 surface ([62], [68], [39]). Given a K3 surface $S$, we denote by

$$
\widetilde{H}(S)=\mathbb{Z} \cdot \mathbb{1} \oplus \mathrm{NS}(S) \oplus \mathbb{Z} \cdot \omega
$$

the algebraic Mukai lattice of $S$, where $\mathbb{1}$ is the fundamental class of $S$ and $\omega$ is the class of a point. An element $r \cdot \mathbb{1}+L+s \cdot \omega$ of $\widetilde{H}(S)$, with $r, s \in \mathbb{Z}$ and $L \in \mathrm{NS}(S)$, is often denoted by $(r, L, s)$. The lattice structure on $\widetilde{H}(S)$ is given by the following Mukai pairing $\langle-,-\rangle$ :

$$
\left\langle(r, L, s),\left(r^{\prime}, L^{\prime}, s^{\prime}\right)\right\rangle=L \cdot L^{\prime}-r s^{\prime}-r^{\prime} s \in \mathbb{Z} .
$$

For be a coherent sheaf $\mathcal{F}$ on $S$, its Mukai vector is defined by

$$
v(\mathcal{F}):=\operatorname{ch}(\mathcal{F}) \sqrt{\operatorname{td}(S)}=\left(\operatorname{rk}(\mathcal{F}), c_{1}(\mathcal{F}), \chi(\mathcal{F})-\operatorname{rk}(\mathcal{F})\right) \in \widetilde{H}(S) .
$$

Fix a polarization $H$ of $X$, we define the Hilbert polynomial of $\mathcal{F}$ as the function

$$
P_{\mathcal{F}}(m)=\operatorname{deg}(\operatorname{ch} \chi(\mathcal{F}(m)) \cdot \operatorname{td}(S)
$$

Let $\alpha_{d}$ be the leading coefficient and define $p(m)=\frac{1}{\alpha_{d}} P_{\mathcal{F}}(m)$. Then $\mathcal{F}$ is GiesekerMaruyama $H$-semistable (stable) if it is pure and

$$
p_{\mathcal{G}}(m) \leq(<) p_{\mathcal{F}}(m) \text {. }
$$

for all non-trivial proper subsheaves $\mathcal{G} \subset \mathcal{F}$.

Definition 4.4 (General polarizations). Let $v \in \widetilde{H}(S)$ be a primitive Mukai vector. We say a polarization $H$ is general with respect to $v$ if every $H$-semistable sheaf is $H$-stable.

For instance, if $v=\left(r, c_{1}, s\right)$, one can easily show that $H$ is general with respect to $v$ if it satisfies the following numerical condition:

$$
\operatorname{gcd}\left(r, c_{1} \cdot H, s\right)=1 .
$$

Given a primitive $e^{4}$ element $v=\left(r, c_{1}, s\right) \in \widetilde{H}(S)$ such that $r>0$ and $\langle v, v\rangle \geq 0$, together with a general ample line bundle $H$ (with respect to $v$ ) on $S$, we consider the moduli space of Gieseker-Maruyama $H$-stable sheaves $\mathcal{F}$ on $S$ with $v(\mathcal{F})=v$, denoted by $\mathcal{M}_{H}(S, v)$. According to the work of Langer [47], $\mathcal{M}_{H}(S, v)$ is a quasiprojective scheme over $k$. Mukai [62] proves that $\mathcal{M}_{H}(S, v)$ admits a symplectic form; when $\operatorname{char}(k)=0$, O'Grady [68] shows that it is an irreducible symplectic variety of dimension $2 n=\langle v, v\rangle+2$ and computes the Hodge structure on the degree two cohomology; Huybrechts [39] shows that it is of $K 3^{[n]}$-deformation type. Over fields of positive characteristic, the following analogous properties of $\mathcal{M}_{H}(S, v)$ hold.

Proposition 4.5 (cf. $[19, \S 2])$. Let $S$ be a smooth projective $K 3$ surface, $H$ an ample line bundle and $v=\left(r, c_{1}, s\right) \in \widetilde{H}(S)$ be a primitive Mukai vector with $r>0$ and $\langle v, v\rangle>0$. If $H$ is general with respect to $v$, then

\footnotetext{
4 that is, $v$ cannot be written as $m v^{\prime}$ for an integer $m \geq 2$ and $v^{\prime}$ another element in $\widetilde{H}(S)$.
} 
(i) $M_{H}(S, v)$ is a smooth projective, irreducible symplectic variety of dimension $2 n=\langle v, v\rangle+2$ over $k$ and it is deformation equivalent to the $n$-th Hilbert scheme $S^{[n]}$.

(ii) The Hodge numbers $h^{i, j}(X):=\operatorname{dim} H^{j}\left(X, \Omega_{X}^{i}\right)$ satisfy

$$
\begin{aligned}
h^{1,0}\left(M_{H}(S, v)\right)=h^{0,1}\left(M_{H}(S, v)\right)=0 & \text { if } p>2 \\
h^{2,0}\left(M_{H}(S, v)\right)=h^{0,2}\left(M_{H}(S, v)\right)=1 & \text { if } p>3 \\
h^{i, j}\left(M_{H}(S, v)\right)=h^{i, j}\left(S^{[n]}\right) & \text { if } p \geq 2 n
\end{aligned}
$$

(iii) When $\ell \neq p$, there is a canonical quadratic form on $H^{2}\left(M_{H}(S, v), \mathbb{Z}_{\ell}(1)\right)$ with values in $\mathbb{Z}\left[\frac{1}{\ell}\right]$. Let $v^{\perp}$ be the orthogonal complement of $v$ in the $\ell$-adic Mukai lattice of $S$. There is an injective isometry

$$
\theta_{v}: v^{\perp} \cap \widetilde{H}(S) \rightarrow \mathrm{NS}\left(M_{H}(S, v)\right),
$$

whose cokernel is a p-primary torsion group. Here $v^{\perp} \cap \widetilde{H}(S)$ is the orthogonal complement of $v$ in $\widetilde{H}(S)$. The discriminant of $\mathrm{NS}(S)$ divides the discriminant of $\mathrm{NS}\left(M_{H}(S, v)\right)$.

(iv) There is an isomorphism

$$
v^{\perp} \otimes K \rightarrow H^{2}\left(M_{H}(S, v), K\right)
$$

between the rational etále (resp. crystalline) cohomology groups, where $K=\mathbb{Q}_{\ell}$ (resp. the fraction field of $W$ ). Here $v^{\perp} \otimes K$ is the orthogonal complement of $v$ in $\oplus H^{i}(S, K)$. It is compatible with the isometry (iii) via the etále (resp. crystalline) cycle class map.

Proof. All the assertions are well-known over characteristic zero fields (cf. [62], [68]). When $\operatorname{char}(k)>0$ and assuming condition (17), the statements $(i),($ iii) and (iv) are proved in [19, Theorem 2.4] via lifting to characteristic zero. But there is no difficulty to extend them to the case $H$ is general. Let us sketch the proof as below.

For part (i), as $H$ is general, Mukai's resut [62] shows that $M_{H}(S, v)$ is a smooth projective variety of dimension $\langle v, v\rangle+2$ if non-empty and it is endowed with a symplectic structure. The non-emptyness of $M_{H}(S, v)$ follows from [92, Theorem $3.16]$.

To show that it has trivial fundamental group and is deformation equivalent to Hilbert scheme of points on K3 surfaces, one can use Proposition 4.3 to find a projective lift

$$
\mathcal{S} \rightarrow \operatorname{Spec} W^{\prime},
$$

of $S$ over some discrete valuation ring $W^{\prime}$ such that both $H$ and $v$ lift to $\mathcal{S}$, denoted by $\mathcal{H} \in \operatorname{Pic}(\mathcal{S})$ and $v$ respectively. We consider the relative moduli space of semistable sheaves on $\mathcal{S}$ over $W^{\prime}$

$$
M_{\mathcal{H}}(\mathcal{S}, v) \rightarrow \operatorname{Spec} W^{\prime} .
$$

Let $\mathcal{S}_{\eta}$ be the generic fiber of $(21)$ and let $\mathcal{H}_{\eta}$ be the restriction of $\mathcal{H}$ to $\mathcal{S}_{\eta}$. Observe that the ample line bundle $\mathcal{H}_{\eta}$ on the generic fiber $\mathcal{S}_{\eta}$ remains general with respect to $v$ ( $c f$. [16, Theorem $4.19(1)]$ ), so the generic fiber of (22) is a smooth (geometrically) irreducible symplectic variety and it is deformation equivalent to $\mathcal{S}_{\eta}^{[n]}$. It follows that $M_{H}(S, v)$ is deformation equivalent to $S^{[n]}$. 
It remains to prove that $M_{H}(S, v)$ has trivial étale fundamental group. This follows from the general fact that the specialization morphism for étale fundamental group is surjective [1, Éxposé X, Corollaire 2.3].

Similarly, one can prove (iii) and (iv) as in [19, Theorem 2.4]. We shall remark that [19] only deal with the étale cohomology groups, but the same argument holds for crystalline cohomology groups as well. The last sentence of (iii) is given in [19, Corollary 2.7].

For part (ii), we only sketch the proof of the last equality in (18) as the rest of the proof is very similar ( $c f$. [19, Proposition 2.5]). In characteristic zero, the assertion follows from Hodge theory. When $k$ has positive characteristic, the well-known result of Deligne-Illusie [25] shows that the Frölicher spectral sequence of $M_{H}(S, v)$ degenerate at $E_{1}$ if $p \geq 2 n$. So it suffices to show the Betti numbers of $M_{H}(S, v)$ is the same as those of the generic fiber $M_{H}\left(\mathcal{S}_{\eta}, v\right)$ of $M_{H}(\mathcal{S}, v) \rightarrow$ Spec $W^{\prime}$. Due to the universal coefficient theorem and integral comparison theorem ( $c f$. [28]), this holds if the $p$-adic cohomology groups of $M_{H}\left(\mathcal{S}_{\eta}, v\right)$ are torsion free. Since $M_{H}\left(\mathcal{S}_{\eta}, v\right)$ is deformation equivalent to the Hilbert scheme $\mathcal{S}_{\eta}^{[n]}$, the torsion freeness then follows from Markman's work [57].

Remark 4.6. One can also consider the moduli space of semistable purely 1dimensional sheaves and a similar result still holds. In particular, if $v=\left(0, c_{1}, s\right)$ satisfying $\langle v, v\rangle=0$ and $\operatorname{gcd}\left(H \cdot c_{1}, s\right)=1$, then every $H$-semistable coherent sheaf is $H$-stable and $M_{H}(S, v)$ is a (non-empty) smooth K3 surface (cf. [16, Proposition 4.20]).

4.3. Moduli spaces of sheaves on supersingular K3 surfaces. Now we specialize to supersingular K3 surfaces. Our first goal is to show in Lemma 4.8 that in the supersingular case, the condition (17) in Proposition 4.5 can always be achieved, up to the twisting of a line bundle, except an extremal case.

We recall two standard auto-equivalences of the derived category $\mathrm{D}^{\mathrm{b}}(S)$ of bounded complexes of coherent sheaves, as well as the corresponding cohomological transforms $(c f .[38])$ :

(1) Let $L$ be a line bundle, then tensoring with $L$ gives an equivalence

$$
-\otimes L: \mathrm{D}^{\mathrm{b}}(S) \rightarrow \mathrm{D}^{\mathrm{b}}(S) .
$$

the corresponding map on cohomology

$$
\exp _{L}: \tilde{H}(S) \rightarrow \tilde{H}(S)
$$

sends a Mukai vector $v=\left(r, c_{1}, s\right)$ to $e^{L} \cdot v=\left(r, c_{1}+r L, s+\frac{r L^{2}}{2}+c_{1} \cdot L\right)$.

(2) Let $E \in \mathrm{D}^{\mathrm{b}}(S)$ be spherical object, that is, $\operatorname{Ext}^{*}(E, E)=H^{*}\left(\mathbb{S}^{2}, k\right)$. There is the spherical twist with respect to $E$ (see $[38, \S 8.1]$ )

$$
T_{E}: \mathrm{D}^{\mathrm{b}}(S) \rightarrow \mathrm{D}^{\mathrm{b}}(S)
$$

and the corresponding cohomological transform

$$
T_{E}: \tilde{H}(S) \rightarrow \tilde{H}(S) .
$$

In particular, when $E=\mathcal{O}_{C}(-1)$ with $C \cong \mathbb{P}^{1}$ a $(-2)$-curve on $S$, we have

$$
T_{E}\left(r, c_{1}, s\right)=\left(r, s_{[C]}\left(c_{1}\right), s\right)
$$

where $s_{[C]}$ is the Mukai refection with respect to the $(-2)$-class $[C] \epsilon$ $\mathrm{NS}(S)$. 
Definition 4.7. Let $\Lambda$ be a lattice. Given an element $x \in \Lambda$ and $m \in \mathbb{Z}^{>0}$, we say $x \cdot \Lambda$ is divisible by $m$ and denote it by $m \mid x \cdot \Lambda$ if $m \mid(x, y)$ for all $y \in \Lambda$. We denote by $m+x \cdot \Lambda$ if $x \cdot \Lambda$ is not divisible by $m$.

Let $S$ be a supersingular K3 surface defined over an algebraically closed field of positive characteristic $p$. A Mukai vector $v=\left(r, c_{1}, s\right) \in \widetilde{H}(S)$ is called coprime to $p$, if $p+r$ or $p+s$ or $p+c_{1} \cdot \mathrm{NS}(S)$.

The following observation says that we do not need to worry about the numerical condition (17) if the K3 surface is supersingular.

Lemma 4.8. Let $S$ be a supersingular K3 surface. If $v \in \tilde{H}(S)$ is a Mukai vector coprime to $p$, then up to tensoring with a line bundle, the numerical condition (17) and hence the conclusions in Proposition 4.5 holds for any ample line bundle $H$.

Proof. As tensoring a sheaf with a line bundle $L$ induces an isomorphism of moduli spaces

$$
M_{H}(S, v) \cong M_{H}\left(S, \exp _{L}(v)\right)
$$

it suffices to prove the existence of line bundle $L$, such that condition (17) is verified by $\exp _{L}(v)$, i.e.

$$
\operatorname{gcd}\left(r,\left(c_{1}+r L\right) \cdot H, s+\frac{r L^{2}}{2}+c_{1} \cdot L\right)=1
$$

We will make use of Proposition 4.1 on the structure of the lattice $\operatorname{NS}(S)$.

Set $q=\operatorname{gcd}\left(r, c_{1} \cdot H\right)$ and we have

$$
\operatorname{gcd}\left(r,\left(c_{1}+r L\right) \cdot H, s+\frac{r L^{2}}{2}+c_{1} \cdot L\right)=\operatorname{gcd}\left(q, s+c_{1} \cdot L\right) .
$$

Let $q_{1}, \cdots, q_{r}$ be the distinct prime numbers dividing $q$. If for any $i$, we could manage to find a line bundle $L_{i} \in \mathrm{NS}(S)$ such that $\operatorname{gcd}\left(q_{i}, s+c_{1} \cdot L_{i}\right)=1$, i.e. $q_{i}+s+c_{1} \cdot L_{i}$, then the line bundle

$$
L:=\sum_{i=1}^{r}\left(a_{i} \prod_{j \neq i} q_{j}\right) \cdot L_{i}
$$

satisfies that $q_{i}+s+c_{1} \cdot L$ for any $i$, or equivalently, $\operatorname{gcd}\left(q, s+c_{1} \cdot L\right)=1$, where $a_{i}$ 's are integers such that $\sum_{i=1}^{r} a_{i} \prod_{j \neq i} q_{j}=1$.

As a result, one can assume that $q=\operatorname{gcd}\left(r, c_{1} \cdot H\right)$ is a prime number. Now, if $q=p$, then by the hypothesis that the Mukai vector $v$ is coprime to $p$, we know that either $p+s$ or there exists $L_{0} \in \operatorname{NS}(S)$ such that $p+c_{1} \cdot L_{0}$. Then we can take $L$ to be the trivial bundle or $L_{0}$ respectively. If $q \neq p$ and assume by absurd that $\operatorname{gcd}\left(q, s+c_{1} \cdot L\right)=q$ for all $L \in \mathrm{NS}(S)$. This means

$$
q \mid s \text { and } q \mid c_{1} \cdot \operatorname{NS}(S) .
$$

Then, note that $\operatorname{NS}(S)$ is a $p$-primary lattice, the class $c_{1}$ has to be divisible by $q$. This is invalid because the vector $v=\left(r, c_{1}, s\right)$ is primitive by our assumption.

Remark 4.9. We shall remark that the exceptional case where the Mukai vector is not coprime to $p$ can only happen when $p \mid \frac{1}{2}\langle v, v\rangle$. This is trivial when $p>2$. When $p=2$, this follows from the fact that $\mathrm{NS}(S)$ is an even lattice of type I in the sense of Rudakov-Safarevic $(c f .[77, \S 2])$. In other words, $M_{H}(S, v)$ is a smooth projective irreducible symplectic variety for any polarization $H$ whenever $M_{H}(S, v) \neq \varnothing$ and $p+\frac{1}{2}\left(\operatorname{dim} M_{H}(S, v)-2\right)$. 
Next, we discuss the birational equivalences between $M_{H}(S, v)$ via changing the stability conditions. The following results is based on Bayer-Macrì's wall-crossing principle in characteristic 0 ( $c f .[7])$.

Theorem 4.10. Let $S$ be a supersingular $K 3$ surface over an algebraically closed field $k$ of characteristic $p>0$. Let $v_{1}, v_{2} \in \tilde{H}(S)$ be two Mukai vectors coprime to $p$ (Definition 4.7). Let $H$ and $H^{\prime}$ be two ample line bundles on $S$. Suppose that $v_{1}$ and $v_{2}$ are differed by a cohomological transform induced by an auto-equivalence of the following form:

(1) tensoring with a line bundle;

(2) spherical twist associated to a line bundle or $\mathcal{O}_{C}(-1)$ for some smooth rational curve $C$ on $S$;

Then $M_{H}\left(S, v_{1}\right)$ is liftably birational to $M_{H^{\prime}}\left(S, v_{2}\right)$ in the sense of Definition 3.25.

Proof. This is indeed a special case of wall-crossing principle for the moduli space of stable complexes on K3 surfaces, which is proved in characteristic zero. Over positive characteristic fields, the wall-crossing principle for K3 surfaces is not completely known. Therefore, we prove the assertion by lifting the K3 surfaces to characteristic 0 .

Write $v_{1}=\left(r, c_{1}(L), s\right)$ and $v_{2}=\left(r^{\prime}, c_{1}\left(L^{\prime}\right), s^{\prime}\right)$ with $L, L^{\prime} \in \operatorname{Pic}(S)$. According to Lemma 4.8, we can assume that $v_{1}$ and $v_{2}$ satisfy the condition (17) without changing the moduli spaces. Suppose $v_{1}$ and $v_{2}$ are differed by a spherical twist $T_{\mathcal{O}_{C}(-1)}$ with $C \cong \mathbb{P}^{1}$, i.e. $v_{2}=T_{\mathcal{O}_{C}(-1)}\left(v_{1}\right)$. As in Proposition 4.5 , there exists a projective lift $\left(\mathcal{S}, \mathcal{H}, \mathcal{H}^{\prime}, \mathcal{L}, \mathcal{L}^{\prime}, \mathcal{C}\right)$ of the 6 -tuples $\left(S, H, H^{\prime}, L, L^{\prime}, C\right)$ over a finite extension $W^{\prime}$ of the Witt ring $W(k)$, which induces projective lifts

$$
M_{\mathcal{H}}\left(\mathcal{S}, v_{1}\right) \rightarrow \operatorname{Spec} W^{\prime}, M_{\mathcal{H}^{\prime}}\left(\mathcal{S}, v_{2}\right) \rightarrow \operatorname{Spec} W^{\prime}
$$

of $M_{H}\left(S, v_{1}\right)$ and $M_{H^{\prime}}\left(S, v_{2}\right)$ respectively as the moduli space of relative stable sheaves on $\mathcal{S}$ over $W^{\prime}$ with given Mukai vectors by Langer's result [47, Theorem 0.2]. Here, by abuse of notations, we keep using $v_{1}=\left(r, c_{1}(\mathcal{L}), s\right)$ and $v_{2}=\left(r^{\prime}, c_{1}\left(\mathcal{L}^{\prime}\right), s^{\prime}\right)$ as Mukai vectors of $\mathcal{S}$.

Let $K^{\prime}$ be the fraction field of $W^{\prime}$. Note that the auto-equivalence $T_{\mathcal{O}_{C}(-1)}$ lifts to $T_{\mathcal{O}_{\mathcal{C}}(-1)}$ and $v_{2}=T_{\mathcal{O}_{\mathcal{C}}(-1)}\left(v_{1}\right)$, then the result [7, Corollary 1.3] (see Remark 4.11) shows that $M_{\mathcal{H}}\left(\mathcal{S}_{\bar{K}}, v_{1}\right)$ is birationally equivalent to $M_{\mathcal{H}^{\prime}}\left(\mathcal{S}_{\bar{K}}, v_{2}\right)$ over $\bar{K}$. Then there exists a discrete valuation ring $W^{\prime \prime}$ as a finite extension of $W^{\prime}$ such that the birational equivalence

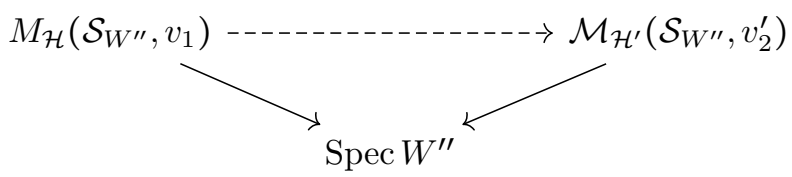

is defined over $W^{\prime \prime}$. Let $\kappa$ be the residue field of $W^{\prime \prime}$ which contains $k$. Then the special fibers of $M_{\mathcal{H}}\left(\mathcal{S}_{W^{\prime \prime}}, v_{i}\right), i=1,2$ over $\operatorname{Spec} W^{\prime \prime}$ is just $M_{H}\left(S_{\kappa}, v_{i}\right)$.

The following argument is then standard. Since the generic fibers of (25) are birational and the special fibers are not ruled, it follows from [58, Theorem 1] that the special fibers $M_{H}\left(S_{\kappa}, v\right)$ and $M_{H^{\prime}}\left(S_{\kappa}, v^{\prime}\right)$ are birationally equivalent over $\kappa$. Finally, as $k$ is algebraically closed and $M_{H}\left(S_{\kappa}, v_{1}\right)=M_{H}\left(S, v_{1}\right) \otimes \kappa$, then $M_{H}\left(S, v_{1}\right)$ and $M_{H^{\prime}}\left(v_{2}\right)$ are birationally equivalent over $k$ as well.

For other cases, the proof is basically the same without much change. 
Remark 4.11. The result [7, Corollary 1.3] is originally stated over complex numbers, but there is no constraint to extend it to any algebraically closed field of characteristic 0 .

Remark 4.12. In general, one may consider the moduli space of Bridgeland stable complexes on K3 surfaces. According to the wall-crossing principle, they are expected to be birational equivalent to the moduli space of stable sheaves considered above. See [60] for more details.

4.4. Hilbert schemes and their birational models. In this subsection, we establish all our conjectures in the introduction for irreducible symplectic varieties that are quasi-liftably birational (Definition 3.25) to Hilbert schemes of K3 surfaces. We will see in the next subsection that almost all moduli spaces of stable sheaves on supersingular K3 surfaces are of this type.

Proposition 4.13 (Birational Hilbert schemes). Let $X$ be an irreducible symplectic variety of dimension $2 n$ over an algebraically closed field $k$. Suppose that $X$ is birational to the Hilbert scheme $S^{[n]}$ for some K3 surface $S$. Then the following are equivalent

(i) $S$ is supersingular.

(ii) $X$ is $2^{\text {nd }}$-Artin supersingular.

(iii) $X$ is $2^{\text {nd }}$-Shioda supersingular.

(iv) $X$ is unirational.

If moreover $X$ is quasi-liftably (Definition 3.25) birational to the Hilbert scheme $S^{[n]}$ for some K3 surface $S$, then the above $(i) \sim(i v)$ are also equivalent to any of the following

$\left(i i^{\prime}\right) X$ is fully Artin supersingular.

(iii') $X$ is fully Shioda supersingular.

(v) The Chow motive of $X$ is of Tate type.

In particular, in this case, the natural epimorphism $\mathrm{CH}^{*}(X)_{\mathbb{Q}} \rightarrow \overline{\mathrm{CH}^{*}}(X)_{\mathbb{Q}}$ is an isomorphism, and they provide, via the cycle class maps, canonical $\mathbb{Q}$-structures for the crystalline cohomology $H_{\mathrm{cris}}^{*}(X / W)_{K}$ and étale cohomology $H_{e ̂ t}^{*}\left(X, \mathbb{Q}_{\ell}\right)$ for all $\ell \neq p$.

Proof. The implications $(i v) \Rightarrow(i i i) \Rightarrow(i i)$ are explained in Remark 2.5 and Theorem 2.8.

For $(i i) \Leftrightarrow(i)$ : recall the notation $W:=W(k), K:=\operatorname{Frac}(W)$ and $H^{*}(X, K):=$ $H_{\text {cris }}^{*}(X / W) \otimes_{W} K$. Suppose that $X$ is birational to $S^{[n]}$ for some K3 surface $S$. Consider the following commutative diagram

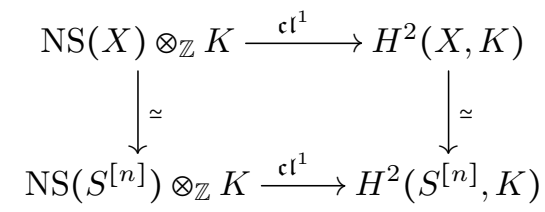

whose vertical arrows are isomorphisms because $X$ and $S^{[n]}$ are K-equivalent ( $c f$. [42], [88], [86]). Hence $X$ is $2^{n d}$-Artin supersingular if and only if $S^{[n]}$ is $2^{n d}$-Artin supersingular, which is again equivalent to the supersingularity of $S$ because we have an isomorphism of $F$-crystals $H^{2}\left(S^{[n]}\right) \simeq H^{2}(S) \oplus W(-1)$.

For $(i) \Rightarrow(i v)$ : The results of [77], [53] and [16] prove Conjecture 1.1, so $(i)$ 
implies that $S$ is unirational. Hence $S^{[n]}$, being rationally dominated by $S^{n}$, is also unirational and so is $X$.

Let us now consider the case where $X$ is quasi-liftably birational to $S^{[n]}$. The implications $(v) \Rightarrow\left(i i i^{\prime}\right) \Rightarrow\left(i i^{\prime}\right)$ are explained respectively in Corollary 2.16 (note that Tate motives are in particular supersingular abelian motives) and in Remark 2.5. As $\left(i i^{\prime}\right)$ is clearly stronger than $(i i)$, it only remains to show $(i) \Rightarrow(v)$. Thanks to Proposition 3.24, two quasi-liftably birational irreducible symplectic varieties have isomorphic Chow motives, hence it suffices to show that the Chow motive of $S^{[n]}$ is of Tate type for a supersingular K3 surface $S$.

To this end, we invoke the following result of de Cataldo-Migliorini [20] on the motivic decomposition of $S^{[n]}$ : in the category of rational Chow motives,

$$
\mathfrak{h}\left(S^{[n]}\right)(n) \simeq \bigoplus_{\lambda \dashv n} \mathfrak{h}\left(S^{|\lambda|}\right)^{\mathfrak{S}_{\lambda}}(|\lambda|)
$$

where $\mathfrak{h}$ is the Chow motive functor, the direct sum is indexed by all partitions of $n$ and for a given partition $\lambda=\left(1^{a_{1}} 2^{a_{2} \cdots} n^{a_{n}}\right)$, its length $|\lambda|:=\sum_{i} a_{i}$ and

$$
\mathfrak{S}_{\lambda}:=\mathfrak{S}_{a_{1}} \times \cdots \times \mathfrak{S}_{a_{n}} .
$$

As a result, we only need to show that the Chow motive of $S$ is of Tate type. However, as $S$ is unirational, there exists a surjective morphism $\widetilde{\mathbb{P}^{2}} \rightarrow S$, where $\widetilde{\mathbb{P}^{2}}$ is a successive blow-up of $\mathbb{P}^{2}$ at points. Therefore $\mathfrak{h}\left(\widetilde{\mathbb{P}^{2}}\right)$ is of Tate type by the blow-up formula. Hence $\mathfrak{h}(S)$, being a direct summand of $\mathfrak{h}\left(\widetilde{\mathbb{P}^{2}}\right)$, must be of Tate type too. All the claimed equivalences are proved.

Finally, the last assertion on cycle class map follows directly from $(v)$

4.5. Relating moduli spaces to Hilbert schemes. The goal of this subsection is to establish main Theorem 1.3 (2). The basic strategy is to exploit the elliptic fibration structure on supersingular K3 surfaces and use Bridgeland's result.

4.5.1. Bridgeland's result. The following is our main tool:

Theorem 4.14 (Bridgeland [17]). Let $\pi: X \rightarrow C$ be a smooth relatively minimal elliptic surface over an algebraically closed field $\bar{K}$. We denote by $f \in \operatorname{NS}(X)$ the fiber class of $\pi$. Given $v=\left(r, c_{1}, s\right) \in \widetilde{H}(X)$ satisfying $r>0$ and assume $\operatorname{gcd}\left(r, c_{1} \cdot f\right)=1$. If $\operatorname{char}(\bar{K})=0$, then there exists an ample line bundle $H$ and $a$ birational morphism

$$
M_{H}(X, v) \rightarrow \operatorname{Pic}^{0}(Y) \times Y^{[n]}
$$

where $Y$ is an smooth elliptic surface. In particular, if $X$ is a K3 surface or an abelian surface, so is $Y$ and the assertion also holds for some liftably birational map (27) when $\operatorname{char}(\bar{K})>0$.

Proof. This theorem is due to Bridgeland [17] over the filed of complex numbers and his proof can be easily generalized to any algebraically closed field of characteristic 0. See also [93, Appendix] for a proof over arbitrary characteristic for elliptic surfaces without non reduced fibers.

We sketch here a simplified proof when $X$ is a K3 surface (resp. an abelian surface), by using the wall-crossing results in [7] and [60], and we refer the reader to $[17,93]$ for more details. As $\operatorname{gcd}\left(r, c_{1} \cdot f\right)=1$, we can set $(a, b)$ be the unique pair of integers satisfying

$$
b r-a\left(c_{1} \cdot f\right)=1
$$


and $0<a<r$. As in [17], there exists an ample line bundle $H \in \operatorname{Pic}(X)$ such that a torsion-free sheaf $\mathcal{F}$ with Mukai vector $v$ is stable if and only if its restriction to the general fiber of $\pi$ is stable. Let

$$
Y:=M_{H^{\prime}}(X,(0, a f, b))
$$

be the fine moduli space of stable sheaves of pure dimension 1 on $X$ with Mukai vector $(0, a f, b)$, and $H^{\prime}$ is a generic ample line bundle defined over $K$. Then $Y$ is a K3 surface (resp. an abelian surface) as well. Bridgeland's work (cf. [17, Theorem 5.3]) essentially shows that the universal Poincáre sheaf on $X \times Y$ induced an equivalence $\Phi$ from $\mathrm{D}^{b}(Y)$ to $\mathrm{D}^{b}(X)$ with $\Phi_{*}(1,0, n)=v$. This enables us to conclude the assertion by using [7, Corollary 1.3].

When $\operatorname{char}(\bar{K})>0$, assume first $X$ is a K3 surface, following the notations above, one can use lifting method to find a K3 surface surface $\mathcal{X}$ over $W^{\prime}$ with special fiber $X_{\kappa}$ such that up to a finite extension of $W^{\prime}$, there is a birational map



where $\mathcal{Y}=M_{\mathcal{H}^{\prime}}(\mathcal{X},(0, a f, b))$. The special fiber $Y_{\kappa}$ of $\mathcal{Y} \rightarrow$ Spec $W^{\prime}$ is exactly $M_{H^{\prime}}\left(X_{\kappa},(0, a f, b)\right)$, which is a smooth K3 surface. Then by taking their reduction, one can get $M_{H}\left(X_{\kappa}, v\right)$ and $\operatorname{Pic}^{0}\left(Y_{\kappa}\right) \times Y_{\kappa}^{[n]}$ are birationally equivalent. This proves the assertion. For abelian surfaces, as the lifting method is still valid (see Proposition 6.4), the same argument allows us to conclude.

4.5.2. Elliptic fibrations. The existence of elliptic fibrations plays an important role in the study of supersingular K3 surfaces, for example in Liedtke's proof of their unirationality [53]. For our purpose, we need a more refined analysis of elliptic fibrations on supersingular K3 surfaces. The first key result is the following.

Theorem 4.15. Let $S$ be a supersingular K3 surface defined over an algebraic closed field $k$ with characteristic $p>0$. Let $v=\left(r, c_{1}, s\right) \in \tilde{H}(S)$ be a Mukai vector coprime to $p$. Then up to changing the Mukai vector $v$ via the auto-equivalences listed in Theorem 4.10, there exists an elliptic fibration $\pi: S \rightarrow \mathbb{P}^{1}$ such that $\operatorname{gcd}\left(r, c_{1} \cdot E\right)=1$, where $E \in \mathrm{NS}(S)$ is the fiber class of $\pi$.

Proof. We proceed the proof by several steps.

Step 0. We claim that it suffices to show that there exists a primitive element $x \in \operatorname{NS}(S)$ such that

$$
x^{2}=0 \text { and } \operatorname{gcd}\left(r, c_{1} \cdot x\right)=1 .
$$

This is because the solution $x$ in (30) gives an effective divisor $E$ on $S$ with $E^{2}=0$ and $c_{1}(E)= \pm x$. If $E$ is base point free, the linear system $|E|$ defines an elliptic fibration $S \rightarrow \mathbb{P}^{1}$ as desired. If $E$ is not base point free, one can find a base point free divisor $E^{\prime}$ with $\left(E^{\prime}\right)^{2}=0$ obtained by taking finitely many Mukai reflections of $E$ with respect to a sequence of $(-2)$ curves $C_{1}, C_{2}, \ldots, C_{n}$ on $S$, i.e. $E^{\prime}=$ $s_{\left[C_{1}\right]} \circ s_{\left[C_{2}\right]} \ldots \circ s_{\left[C_{n}\right]}(E)$. Then we set

$$
v^{\prime}=T_{\mathcal{O}_{C_{1}}(-1)} \circ T_{\mathcal{O}_{C_{2}}(-1)} \ldots T_{\mathcal{O}_{C_{n}}(-1)}(v) .
$$


The Mukai vector $v^{\prime}$ and the elliptic fibration $S \rightarrow \mathbb{P}^{1}$ induced by $\left|E^{\prime}\right|$ thus satisfy the desired condition.

Step 1. We reduce to the case $c_{1}$ is primitive. First, if the vector $\left(r, c_{1}\right) \in \mathbb{Z} \oplus \operatorname{NS}(S)$ is primitive and $c_{1}$ is not primitive, then $c_{1}+r L$ will be primitive for some $L \in \operatorname{NS}(S)$. This means that we can replace $v$ by the Mukai vector $v^{\prime}=\exp _{L}(v)$. Similarly, if $\left(c_{1}, s\right)$ is primitive, one can get $v^{\prime}=T_{L}(v)$ satisfying the condition for some line bundle $L$.

Suppose now the vectors $\left(r, c_{1}\right)$ and $\left(c_{1}, s\right)$ are both non-primitive respectively, we can write

$$
r=q_{1} r^{\prime}, c_{1}=q_{1} q_{2} c_{1}^{\prime}, s=q_{2} s^{\prime},
$$

such that $\left(r^{\prime}, q_{2} c_{1}^{\prime}\right)$ and $\left(q_{1} c_{1}^{\prime}, s^{\prime}\right)$ are primitive, where $r^{\prime}, q_{i}, s^{\prime} \in \mathbb{Z}$ are non-zero integers and $c_{1}^{\prime} \in \operatorname{NS}(S)$. Then what we need is the following: find $L \in \operatorname{NS}(S)$ and $L^{2}=0$ such that the vector

$$
\left(c_{1}+r L, s+\frac{r L^{2}}{2}+c_{1} \cdot L\right)=\left(q_{1}\left(q_{2} c_{1}^{\prime}+r^{\prime} L\right), s+c_{1} \cdot L\right)
$$

is primitive because one can replace $v$ by $\exp _{L}(v)$ and the same argument as above works. To see the existence of such $L$, note that

$$
\left(q_{1}, s+c_{1} \cdot L\right)=\left(q_{1}, q_{2}\left(s^{\prime}+q_{1} c_{1}^{\prime} \cdot L\right)\right)
$$

is always primitive, it suffices to find a square zero element $L$ such that the class $q_{2} c_{1}^{\prime}+r^{\prime} L$ is primitive. This can be easily achieved because $\left(r^{\prime}, q_{2} c_{1}^{\prime}\right) \in \mathbb{Z} \oplus \operatorname{NS}(S)$ is primitive by our assumption and the natural basis of $\operatorname{NS}(S)$ contains square zero element (See Proposition 4.1).

Step 2. Let us first assume that $p+r$. Suppose $r$ is a prime number. Recall that $\operatorname{NS}(S)$ is isomorphic to the lattice $-\Lambda_{\sigma(S)}$ given in Proposition 4.1, we may have two cases as below:

(1) If $\Lambda_{\sigma(S)}$ contains a hyperbolic lattice $U$, we denote by $\left\{f_{1}, f_{2}\right\}$ the natural basis of $U$ satisfying $f_{1}^{2}=f_{2}^{2}=0$ and $f_{1} \cdot f_{2}=1$. We could find a basis of $\operatorname{NS}(S)$ of the form the

$$
f_{1}, f_{2}, v-\frac{\langle v, v\rangle}{2} f_{1}+f_{2} v
$$

for some $v \in U^{\perp}$. Note that every element in (33) is square zero, so if there is no solution of (30), we must have $r \mid c_{1} \cdot \mathrm{NS}(S)$. However, as $c_{1}$ is primitive and the lattice $\operatorname{NS}(S)$ is $p$-primary, it forces $r=p$ which contradicts to our assumption.

(2) If $\Lambda_{\sigma(S)}$ contains $U(p)$ with basis $\left\{f_{1}, f_{2}\right\}$ satisfying $f_{i}^{2}=0, f_{1} \cdot f_{2}=p$, the primitive square zero elements

$$
f_{1}, f_{2}, p v-\frac{p\langle v, v\rangle}{2} f_{1}+f_{2}
$$

can also form a basis of the $p$-primary sublattice

$$
U(p) \oplus p\left(U(p)^{\perp}\right) \subseteq \mathrm{NS}(S) .
$$

Then the same argument shows that there must exists $x \in U(p) \oplus p\left(\left(U(p)^{\perp}\right)\right.$ satisfying (30).

Combining (1) and (2), we prove the case when $r$ is a single prime. In general, for any positive integer $r$, let $\Xi \subseteq \mathbb{Z}$ be the collection of all prime factors of $r$. Let $X$ be the projective quadric hypersurface over $\mathbb{Z}$ defined by $x^{2}=0$, which is geometrically 
integral. We can view the desired element $x \in \mathrm{NS}(S)$ as a rational point in $X(\mathbb{Z})$. By weak approximation on quadrics ( $c f .[75, \S 7.1$ Corollary 1]), the diagonal map

$$
X(\mathbb{Z}) \rightarrow \prod_{q \in \Xi} X\left(\mathbb{Z}_{q}\right)
$$

is dense if $X(\mathbb{Z}) \neq \varnothing$. Set

$$
U_{q}=\left\{x \in X\left(\mathbb{Z}_{q}\right) \mid x \cdot c_{1} \in \mathbb{Z}_{q}^{\times}\right\} \subseteq X\left(\mathbb{Z}_{q}\right) .
$$

and we know that $U_{q}$ is an open subset of $X\left(\mathbb{Z}_{q}\right)$ if $U_{q} \neq \varnothing$. From the discussion above, $U_{q}$ is non-empty for each $q \in \Xi$ and hence it is an open dense subset. Thus, there exists $x \in X(\mathbb{Z})$ whose image via (35) lies in $\prod_{q \in \Xi} U_{q}$. Then we have $\operatorname{gcd}(x$. $\left.c_{1}, q\right)=1$ for all $q \in \Xi$ from the construction, which proves the assertion.

Next, if $p+s$, we can replace $v$ by $T_{\mathcal{O}_{S}}(v)$. Then the same argument applies to $T_{\mathcal{O}_{S}}(v)$.

Step 3. Finally, if $p \mid \operatorname{gcd}(r, s)$, then we have $p+c_{1} \cdot \operatorname{NS}(S)$ by $(*)$. As in Step 2, we only need to show $U\left(\mathbb{Z}_{q}\right)$ is not empty for all $q \in \Sigma$. When $q \neq p$, it is easy to see $U\left(\mathbb{Z}_{q}\right) \neq \varnothing$ via the same analysis.

When $q=p$, note that $p+c_{1} \cdot \operatorname{NS}(S)$, the argument in Step 2 actually shows that there exists an element $x$ in (33) or (34) such that $p+c_{1} \cdot x$. This implies that $U_{p}$ is non-empty and hence proves the assertion.

4.5.3. Birationality to Hilbert schemes. The result above yields the birational equivalences between (almost) all moduli spaces of sheaves on a supersingular K3 surface.

Theorem 4.16. Let $S$ be a supersingular K3 surface defined over an algebraic closed field $k$ with characteristic $p>0$. Let $v=\left(r, c_{1}, s\right) \in \tilde{H}(S)$ be a Mukai vector coprime to $p$. Then the moduli space $M_{H}(S, v)$ is quasi-liftably birational to $S^{[n]}$.

Proof. By Theorem 4.10 and Theorem 4.15, there exists $v^{\prime}=\left(r^{\prime}, c_{1}(L), s^{\prime}\right) \in \tilde{H}(S)$ for some $r^{\prime}, s^{\prime} \in \mathbb{Z}$ and $L \in \operatorname{Pic}(S)$ and an elliptic fibration $\pi: S \rightarrow \mathbb{P}^{1}$ of $S$ such that $M_{H}(S, v)$ is quasi-liftably birational to $M_{H}\left(S, v^{\prime}\right)$ and $\operatorname{gcd}\left(r^{\prime}, c_{1}(L) \cdot E\right)=1$ where $E$ is the fiber class of $\pi$.

As in the proof of Theorem 4.10, up to an algebraically closed base field extension $\kappa / k$, we can take a projective lift $(\mathcal{S}, \mathcal{H}, \mathcal{L}, \mathcal{E})$ of the 4 -tuple $\left(S_{\kappa}, H, L, E\right)$ over some discrete valuation ring $W^{\prime}$ of characteristic zero and $\mathcal{H}, \mathcal{L}, \mathcal{E} \in \operatorname{Pic}\left(\mathcal{S} / W^{\prime}\right)$. By considering the relative moduli space of stable sheaves, these data gives rise to a lift

$$
M_{\mathcal{H}}(\mathcal{S}, v) \rightarrow \operatorname{Spec} W^{\prime}
$$

of $M_{H}\left(S_{\kappa}, v\right)$ over $W^{\prime}$. Let $K^{\prime}$ be the fraction field of $W^{\prime}$ and $\mathcal{S}_{K^{\prime}}$ be the generic fiber of $\mathcal{S} \rightarrow$ Spec $W^{\prime}$. By our construction, the linear system $|\mathcal{E}|$ defines an elliptic fibration

$$
\pi: \mathcal{S}_{K^{\prime}} \rightarrow \mathbb{P}_{K^{\prime}}^{1}
$$

whose fiber class $f \in \mathrm{NS}\left(\mathcal{S}_{K^{\prime}}\right)$ of $\pi$ satisfies the condition $\operatorname{gcd}\left(r, c_{1} \cdot f\right)=1$. Then Bridgeland's result Theorem 4.14, combined with Theorem 4.10, shows that up to a finite extension of $W^{\prime}$, there exists a smooth K3 surface $\mathcal{S}^{\prime}$ defined over $W^{\prime}$ with smooth special fiber $S_{\kappa}^{\prime}$, such that the generic fiber $M_{H}\left(S_{K^{\prime}}, v\right)$ of (36) is birational equivalent to the Hilbert scheme $\mathcal{S}_{K^{\prime}}^{\prime[n]}$ over $K^{\prime}$. Then the standard reduction argument implies the existence of birational equivalence

$$
M_{H}\left(S_{\kappa}, v\right) \rightarrow\left(S_{\kappa}^{\prime}\right)^{[n]}
$$


on the special fiber.

At last, note that the two K3 surfaces $S_{\kappa}$ and $S_{\kappa}^{\prime}$ are derived equivalent, then they have to be isomorphic because supersingular K3 surfaces have no FourierMukai partners ( $c f$. [50, Theorem 1.1]). This concludes our assertion.

Proof of Theorem 1.3. The assertion (1) follows from Theorem 4.5 (i) and (iv).

(2) follows from Theorem 4.16 and Proposition 4.13.

Proof of Corollary 1.4. The equivalence follows from the combination of Proposition 4.13 and Theorem 4.16.

\section{Moduli SPACES OF TWISTED SHEAVES ON K3 SURfaCeS}

In this section, we extend our results in $\S 4$ to the moduli spaces of twisted sheaves on K3 surfaces.

5.1. Twisted sheaves on K3 surfaces. We mainly follow [51] and [16] to review the basic facts of twisted sheaves on K3 surfaces. Let $S$ be a K3 surface over $k$ and let $\mathscr{S} \rightarrow S$ be a $\mu_{m}$-gerbe over $S$. This corresponds to a pair $(S, \alpha)$ for some $\alpha \in H_{f l}^{2}\left(S, \mu_{m}\right)$, where the cohomology group is with respect to the flat topology. There is a Kummer exact sequence

$$
1 \rightarrow \mu_{m} \rightarrow \mathbb{G}_{m} \stackrel{x \mapsto x^{m}}{\longrightarrow} \mathbb{G}_{m} \rightarrow 1
$$

in flat topology and it induces a surjective map

$$
H_{f l}^{2}\left(S, \mu_{m}\right) \rightarrow \operatorname{Br}(S)[m] .
$$

Definition 5.1 ( $B$-fields). For a prime $\ell \neq p$, an $\ell$-adic $B$-field on $S$ is an element $B \in H_{e ̂ t}^{2}\left(S, \mathbb{Q}_{\ell}(1)\right)$. It can be written as $\alpha / \ell^{n}$ for some $\alpha \in H_{\text {ét }}^{2}\left(S, \mathbb{Z}_{\ell}(1)\right)$ and $n \in \mathbb{Z}$. We associate a Brauer class $\left[B_{\alpha}\right]$ as the image of $\alpha$ under the following composition of natural maps

$$
H_{e ́ t}^{2}\left(S, \mathbb{Z}_{\ell}(1)\right) \rightarrow H^{2}\left(S, \mu_{\ell^{n}}\right) \rightarrow \operatorname{Br}(S)\left[\ell^{n}\right], \text { if } \ell+p .
$$

A crystalline $B$-field is an element $B=\frac{\alpha}{p^{n}} \in H_{\text {cris }}^{2}(S / W) \otimes K$ with $\alpha \in H_{\text {cris }}^{2}(S / W)$, so that the projection of $\alpha$ in $H_{\text {cris }}^{2}\left(S / W_{n}(k)\right)$ lies in the image of the map

$$
H_{f l}^{2}\left(S, \mu_{p^{n}}\right) \stackrel{d \log }{\longrightarrow} H_{\text {cris }}^{2}\left(S / W_{n}(k)\right)
$$

See [40, I.3.2, II.5.1] for the details of the map (39). Then we can associate a $p^{n}$-torsion Brauer class $\left[B_{\alpha}\right]$ via the map (38).

Let us write $B=\frac{\alpha}{r}$ as either a $\ell$-adic or crystalline $B$-field of $\mathscr{S} \rightarrow S$, we define the twisted Mukai lattice as

$$
\widetilde{H}(\mathscr{S})=\left\{\begin{array}{l}
e^{a / r}\left(\tilde{H}(S) \otimes \mathbb{Z}_{\ell}\right) ; \text { if } p+m \\
e^{a / r}\left(\tilde{H}_{\text {cris }}(S / W)\right) ; \text { if } m=p^{n}
\end{array}\right.
$$

under the Mukai pairing (15).

Definition 5.2. An $\mathscr{S}$-twisted sheaf $\mathcal{F}$ on $\mathscr{S}$ is an $\mathcal{O}_{\mathscr{S}}$-modulo compatible with the $\mu_{m}$-gerbe structure ( $c f$. [49, Def 2.1.2.4]). With the notation as above, the Mukai vector of $\mathcal{F}$ is defined as

$$
v^{\alpha / r}(\mathcal{F})=e^{a / r} \operatorname{ch} \mathscr{S}(\mathcal{F}) \sqrt{\operatorname{td}_{S}} \in \operatorname{CH}^{*}(S, \mathbb{Q}) .
$$


where $e^{a / r}=\left(1, a / r, \frac{(a / r)^{2}}{2}\right)$ and $\operatorname{ch}_{\mathscr{S}}(\mathcal{F})$ is the twisted Chern character of $\mathcal{F}(c f$. [51, 3.3.4]). It can be also viewed as an element in the twisted Mukai lattice $\widetilde{H}(\mathscr{S})$ via the corresponding cycle class map.

Similarly as the case of untwisted sheaves, we say that $H$ is general with respect to $v$ if every $H$-semistable twisted sheaf is $H$-stable.

\subsection{Moduli spaces of twisted sheaves.}

Definition 5.3 ( $c f$. [49]). Fix a polarization $H$ on a K3 surface $S$, the moduli stack $\mathscr{M}_{H}(\mathscr{S}, v)$ of $\mathscr{S}$-twisted sheaves with Mukai vector $v$ is the stack whose objects over a $k$-scheme $T$ are pairs $(\mathcal{F}, \phi)$, where $\mathcal{F}$ is a $T$-flat quasi-coherent twisted sheaf of finite presentation and $\phi: \operatorname{det} \mathcal{F} \rightarrow \mathcal{O}(D)$ is an isomorphism of invertible sheaves on $X$, such that for every geometric point $t \rightarrow T$, the fiber sheaf $\mathcal{F}_{t}$ has Mukai vector $v$ and endomorphism ring $k(t)$.

The first result is

Theorem 5.4. Assume that $\operatorname{char}(k)=p>0$ and $v=\left(r, c_{1}, s\right)$ satisfies $\langle v, v\rangle \geq 0$ and $r>0$. If $H$ is general with respect to $v$, the moduli stack $\mathscr{M}_{H}(\mathscr{S}, v)$ has a smooth and projective coarse moduli space $M_{H}(\mathscr{S}, v)$ of dimension $\frac{\langle v, v\rangle}{2}+1$ if non-empty. The coarse moduli space $M_{H}(\mathscr{S}, v)$ is an irreducible symplectic variety and there exists a canonical quadratic forms on the Néron-Severi group $\operatorname{NS}\left(M_{H}(\mathscr{S}, v)\right)$ such that there is an injective isometry

$$
\left(v^{\perp}\right) \cap \widetilde{H}(\mathscr{S}) \rightarrow \mathrm{NS}\left(M_{H}(\mathscr{S}, v)\right) \otimes R .
$$

where $R=\mathbb{Z}_{\ell}$ or $W$ depending on $\mathscr{S}$ as in (40). Moreover, there is an isomorphism

$$
v^{\perp} \otimes K \rightarrow H_{\text {cris }}^{2}\left(M_{H}(\mathscr{S}, v) / K\right)
$$

as $F$-isocrystals. Here we regard $v$ as an element in $\oplus H^{i}(S / K)$ and $v^{\perp} \otimes K$ is the orthogonal complement.

Proof. Everything is known in characteristic 0 by Yoshioka [92, Theorem 3.16; Theorem 3.19]. Similar to the untwisted case, as $H$ is general, all the assertion can be proved by lifting to characteristic 0 (see [19, Theorem 2.4]). We also refer to [16] for the case where $S$ is supersingular.

Let us consider the case where $S$ is supersingular. By [5], we know that the Brauer group $\operatorname{Br}(S)$ is of $p$-torsion. In this case, there is an explicit description of twisted Mukai lattice in [16]. This enable us to give a sufficient condition for $M_{H}(\mathscr{S}, v)$ to be smooth and projective. The following result is analogous to Lemma 4.8 .

Proposition 5.5. Suppose $S$ is supersingular. If the Mukai vector $v$ is coprime to $p$ with $r>0$ and $\langle v, v\rangle \geq 0$, the coarse moduli space $M_{H}(\mathscr{S}, v)$ is an irreducible symplectic variety for any ample polarization $H$.

Proof. The non-emptyness again follows from [92, Theorem 3.16] (See also [16, Proposition 4.20]). It suffices to show that $H$ is general with respect to $v$. Since $v$ is coprime to $p$, up to change $c_{1}$ by twisting a line bundle, we know that $H$ and $v$ satisfy the numerical condition (17) by using the same argument in the proof of Lemma 4.8 . 
5.3. From moduli spaces of twisted sheaves to those of untwisted ones. We show all our conjectures in the introduction for most moduli spaces of twisted sheaves on K3 surfaces. The key result is Theorem 5.9, which shows that the moduli space $M_{H}(\mathscr{S}, v)$ of twisted sheaves on $\mathscr{S}$ is quasi-liftably birational to some moduli space of untwisted sheaves. To start, we have

Lemma 5.6. Assume that $M_{H}(\mathscr{S}, v)$ is an irreducible symplectic variety. Then it is supersingular if and only if $S$ is supersingular.

Proof. Similar as the untwisted case, this follows from the isomorphism (42).

By Lemma 5.6, we are reduced to consider the moduli space of (semi)-stable twisted sheaves on supersingular K3 surfaces. Let $\mathrm{D}^{(1)}(\mathscr{S})$ be the bounded derived category of twisted sheaves on $\mathscr{S}$. If the gerbe $\mathscr{S} \rightarrow S$ is trivial, then $\mathrm{D}^{(1)}(\mathscr{S})=$ $\mathrm{D}^{b}(S)$. The following result shows that every twisted supersingular K3 surface is derived equivalent to a untwisted supersingular K3 surface, provided that the Artin invariant is less than 10 .

Theorem 5.7 (Untwisting). If $\sigma(S)<10$, there is a Fourier-Mukai equivalence from $\mathrm{D}^{(1)}(\mathscr{S})$ to $\mathrm{D}^{b}\left(S^{\prime}\right)$ for some supersingular K3 surface $S^{\prime}$.

Proof. First, we claim that there exists primitive vectors $\tau \in \tilde{H}(\mathscr{S})$ and $w \in \tilde{H}(\mathscr{S})$ satisfying the condition

$$
\tau^{2}=0 \text { and } p+\tau \cdot w .
$$

Assuming this, we consider the moduli space $\mathscr{M}_{H}(\mathscr{S}, \tau)$ for an ample line bundle $H$. By [16, Theorem 4.19], $\mathscr{M}_{H}(\mathscr{S}, \tau)$ is a $\mathbb{G}_{m}$-gerbe over a supersingular K3 surface $S^{\prime}=M_{H}(\mathscr{S}, \tau)$ if non-empty. The universal sheaf induces a Fourier-Mukai equivalence

$$
\Phi: \mathrm{D}^{(1)}(\mathscr{S}) \rightarrow \mathrm{D}^{(-1)}\left(\mathscr{M}_{H}(\mathscr{S}, \tau)\right),
$$

So it suffices to show the gerbe $\mathscr{M}_{H}(\mathscr{S}, \tau) \rightarrow S^{\prime}$ is trivial. By [16, Theorem 4.19 (3)], this is equivalent to find a vector $w \in \tilde{H}(\mathscr{S})$ such that $\tau \cdot w$ is coprime to $p$. Thus we can conclude our assertion.

Now we start to prove the claim. Write $\alpha=\alpha_{0}+L$ for some $L \in \operatorname{NS}(S)$ and $\left[\alpha_{0}\right]$ the image of $\alpha_{0}$ in $\operatorname{Br}(S)$. For a vector $w \in \tilde{H}(\mathscr{S})$, we can write

$$
w=\left\{\begin{array}{l}
\left(x, \frac{x L}{p}+y D, \frac{x L^{2}}{2 p^{2}}+\frac{y}{p} D \cdot L+z\right), \text { if }\left[\alpha_{0}\right]=0 ; \\
\left(x p, x L+y D, \frac{x L^{2}}{2 p}+\frac{y}{p} D \cdot L+z\right), \text { if }\left[\alpha_{0}\right] \neq 0 ;
\end{array}\right.
$$

for some $D \in \operatorname{NS}(S)$ and $x, y, z \in \mathbb{Z}$ (cf. [16, Proposition 3.60]). Then we have two possibilities:

(a). If $L \cdot \mathrm{NS}(S)$ is not divisible by $p$, then we can find a primitive element $E \in \operatorname{NS}(S)$ such that $E^{2}=0$ and $p+L \cdot E$. Then we take

$$
\tau=(0,[E], s) \text { and } w=\left(p, L, \frac{L^{2}}{2 p}\right) .
$$

It follows that $\tau \cdot w=L \cdot E-s p$ is coprime to $p$.

(b). If $p \mid L \cdot \mathrm{NS}(S)$, then we can take $\tau=\left(p, c_{1}, s\right)$ with $c_{1}^{2}=2 p s$ and choose $w=\left(0, D, \frac{D \cdot L}{p}\right)$ for some $D$ with $p+c_{1} \cdot D$. It follows that

$$
\tau \cdot w=c_{1} \cdot D-D \cdot L
$$


is coprime to $p$. The existence of $c_{1}$ and $D$ can be also deduced from Proposition 4.1. When $p>2$, as $\sigma(S)<10$ and $\operatorname{NS}(S)$ contains a hyperbolic lattice $U$, we can let $f_{i}, i=1,2$ be the standard basis of $U$ and take

$$
c_{1}=f_{1}+p f_{2} \text { and } D=f_{2}
$$

as desired.

When $p=2$, this is more complicated. The lattice $\operatorname{NS}(S)$ contains $U$ only when $\sigma(S)$ is odd. If $\sigma(S)$ is even, $\mathrm{NS}(S)$ contains $U(2)$ as a direct summand instead. We claim that there exist an element $y \in(U(2))^{\perp}$ such that $y^{2}=-4$ and $2+y \cdot \operatorname{NS}(S)$. Assuming this, we can pick $x \in U(2)$ with $x^{2}=4$ and $c_{1} \in \mathrm{NS}(S)$ with $2+c_{1} \cdot y$, one can easily see that the classes $D=x-y$ and $c_{1}$ are as desired.

The proof of the claim is due to the explicit description of $\operatorname{NS}(S)$. For instance, if $\sigma(S)=8$, Rudakov and Safarevic [77] showed that $\Lambda_{\sigma(S)}$ is isomorphic to

$$
U(2) \oplus D_{4}^{\oplus 3} \oplus E_{8}(2),
$$

where $D_{4}$ and $E_{8}$ are root lattices defined by the corresponding Dynkin diagram. There certainly exist some element $y^{\prime} \in D_{4}$ with $\left(y^{\prime}\right)^{2}=2$ and $2+y^{\prime} \cdot D_{4}$. Then we can select $y=\left(y^{\prime}, y^{\prime}\right) \in D_{4}^{\oplus 2}$ with $y^{2}=4$ and $2+y \cdot D_{4}^{\oplus 2}$ which automatically satisfies the conditions. Similar analysis holds when $\sigma(S)=2,4$ and 6 . Here we omit the details and left it to the readers.

Remark 5.8. Theorem 5.7 can be viewed as a converse of [16, Proposition 5.15], which shows every supersingular K3 surface is derived equivalent to a supersingular twisted K3 surface with Artin invariant less than 10.

Theorem 5.9. Let $v$ be a Mukai vector which is coprime to $p$. If the Artin invariant $\sigma(S)<10$, then $M_{H}(\mathscr{S}, v)$ is quasi-liftably birational to $M_{H^{\prime}}\left(S^{\prime}, v^{\prime}\right)$ for some supersingular K3 surface $S^{\prime}, v^{\prime} \in \tilde{H}\left(S^{\prime}\right)$ a Mukai vector which is coprime to $p$ and $H^{\prime} \in \operatorname{Pic}\left(S^{\prime}\right)$ an ample line bundle.

Proof. By assumption, as in the proof of Theorem 5.7, we can find $\tau \in \widetilde{H}(\mathscr{S})$ with $\tau^{2}=0$ such that there is a Fourier-Mukai equivalence

$$
\Phi: \mathrm{D}^{(1)}(\mathscr{S}) \rightarrow \mathrm{D}^{b}\left(S^{\prime}\right)
$$

where $S^{\prime}=M_{H}(\mathscr{S}, \tau)$. As before, we prove the birational equivalence from the wall-crossing principle. As this remain unknown over positive characteristic fields, we proceed the proof by lifting to characteristic 0 . As in Theorem 5.4 (See also [16, Theorem 4.19]), we take a projective lift

$$
\mathscr{S}_{W} \rightarrow \mathcal{S}_{W}, \mathcal{H} \in \operatorname{Pic}(\mathcal{S}), \tau_{W} \in \tilde{H}\left(\mathscr{S}_{W}\right) .
$$

of the triple $\left(\mathscr{S}_{W} \rightarrow S, H, \tau\right)$ over $W$ for some discrete valuation ring $W$. Consider the relative moduli space

$$
\mathscr{M}_{\mathcal{H}}\left(\mathscr{S}, \tau_{W}\right) \rightarrow \operatorname{Spec}(W)
$$

whose special fiber is the (untwisted) supersingular K3 surface $M_{H}(S, \tau)$ shown in Theorem 5.7. Let

By [16, Theorem 4.19], we have a Fourier-Mukai equivalence

$$
\Phi: \mathrm{D}^{(1)}\left(\mathscr{S}_{\eta}\right) \rightarrow \mathrm{D}^{(1)}\left(\mathscr{M}_{\mathcal{H}}\left(\mathscr{S}_{\eta}, \tau_{\eta}\right)\right) .
$$

for the generic fiber of (45) and (46). 
Now we can apply Bayer-Macri's wall-crossing theorem to get a birational map between the coarse moduli spaces

$$
M_{\mathcal{H}}\left(\mathscr{S}_{\eta}, v\right) \rightarrow M_{\mathcal{H}^{\prime}}\left(\mathscr{S}_{\eta}^{\prime}, v^{\prime}\right)
$$

after possibly taking a finite extension of $W$, where $v^{\prime}=\Phi_{*}(v)$. Then we can conclude the assertion by taking the reduction.

Finally, we have to check that the Mukai vector $v^{\prime}$ is coprime to $p$. This is clear as the Fourier-Mukai transform does not change the divisibility of Mukai vectors.

Corollary 5.10. If the Artin invariant $\sigma(S)<10$, then the moduli space $M_{H}(\mathscr{S}, v)$ is unirational and has Chow motive of Tate type. In particular, the same statements in Theorem 1.3 and Corollary 1.4 hold for such moduli spaces.

Proof. As quasi-liftably birational irreducible symplectic varieties have isomorphic Chow motive by Proposition 3.24, Theorem 5.9 allows us to reduce to the untwisted case, namely, Theorem 1.3 and Corollary 1.4, which is proved in $\S 4$.

Remark 5.11. When the Artin invariant $\sigma(S)=10$, the gerbe $\mathscr{S} \rightarrow S$ is no longer derived equivalent to any untwisted supersingular K3 surface because of the constraint of Artin invariants. It remains open whether $M_{H}(\mathscr{S}, v)$ is unirational.

\section{Generalized Kummer varieties}

In this section, we shall prove all our conjectures (§1.3) in the introduction, except the unirationality conjecture, for generalized Kummer varieties.

6.1. Preliminaries on abelian surfaces over positive characteristic. Let $A$ be an abelian surface defined over an algebraically closed field $k$ of positive characteristic $p$. For $n \in \mathbb{Z}$, consider the multiplication by $n$ map

$$
n_{A}: A \rightarrow A,
$$

which is separable if and only if $p+n$. When $p \mid n$, the inseparable morphism factors through the absolute Frobenius map $F: A \rightarrow A$. Denote by $A[n]$ the kernel of $n_{A}$. The Newton polygon of $H^{1}(A)$ can be computed via the $k$-rational points of $A[p]$ and in particular,

- $A$ is ordinary if and only if $A[p](k) \cong(\mathbb{Z} / p \mathbb{Z})^{2}$

- $A$ is ( $1^{s t}$-Artin) supersingular if and only if $A[p](k)=\{0\}$.

When $A$ is supersingular, the Néron-Severi group $\operatorname{NS}(A)$ equipped with the intersection form, is an even lattice of rank 6 with discriminant equal to $p^{2}$ or $p^{4}$ (one could say that the Artin invariant is 1 or 2). As before, one can use the work of Rudakov-Šaferevič [77] and Shimada [80] to have a classification of such lattices and yield the following description of the Néron-Severi lattice of supersingular abelian surfaces, in a parallel way to Proposition 4.1.

Proposition 6.1 ([77, §2], [80]). Let $A$ be a supersingular abelian surface defined over an algebraically closed field of positive characteristic $p$.

If $\operatorname{disc}(\mathrm{NS}(A))=p^{2}$, then the lattice $\operatorname{NS}(A)$ is isomorphic to $-\Lambda_{1}$, with

$$
\Lambda_{1}= \begin{cases}U \oplus D_{4}, & \text { if } p=2, \\ U \oplus V_{4,2}^{(p)}, & \text { if } p \equiv 3 \bmod 4, \\ U \oplus H^{(p)}, & \text { if } p \equiv 1 \bmod 4,\end{cases}
$$


If $\operatorname{disc}(\mathrm{NS}(A))=p^{4}$, then the lattice $\mathrm{NS}(A)$ is isomorphic to $-\Lambda_{2}$, with

$$
\Lambda_{2}= \begin{cases}U(p) \oplus D_{4}, & \text { if } p=2, \\ U(p) \oplus H^{(p)}, & \text { otherwise }\end{cases}
$$

Here, $U, V_{4,2}^{(p)}$ and $H^{(p)}$ are the lattices defined in Proposition 4.1.

As in the case of K3 surfaces, we obtain

Corollary 6.2 (cf. [53, Proposition 3.9]). Let A a supersingular abelian surface defined over an algebraically closed field of positive characteristic $p$. Then $A$ admits an elliptic fibration with a section if $\operatorname{disc}(\mathrm{NS}(A))=p^{2}$ and an elliptic fibration with a multisection of degree $p$ when $\operatorname{disc}(\mathrm{NS}(A))=p^{4}$.

A simple observation is the following

Lemma 6.3. Any supersingular abelian surface admits a principal polarization.

Proof. Let $A$ be a supersingular abelian surface. By Proposition 6.1, we know that the Néron-Severi lattice $\mathrm{NS}(A)$ has two possibilities $-\Lambda_{1}$ or $-\Lambda_{2}$ given by (49) and (50). In any case, we have a line bundle $L$ with $\left(L^{2}\right)=2$. Replacing $L$ by its inverse if necessary, we see that $L$ is ample. Since $h^{0}(A, L)=\chi(A, L)=\frac{\left(L^{2}\right)}{2}=1$, it is a principal polarization.

Now we turn to the liftability of supersingular abelian surfaces. Lifting polarized abelian surfaces to characteristic 0 has been established by Mumford, Norman and Oort (cf. [65]). For our purpose, we need to slightly generalize their results to the lifting of abelian surface together with several line bundles. The following result is analogous to Proposition 4.3.

Proposition 6.4. Let $A$ be an abelian surface over a perfect field $k$ of characteristic $p>0$. Suppose $L_{1}, L_{2}$ are two line bundles on $A$ and $L_{1}$ is a separable polarization. Then there exists a complete discrete valuation ring $W^{\prime} \supseteq W(k)$, finite over $W(k)$ and a projective lift of $A$

$$
\mathcal{A} \rightarrow \operatorname{Spec}\left(W^{\prime}\right)
$$

such that rank $\operatorname{NS}\left(\mathcal{A}_{\eta}\right)=2$ and the image of the specialization map

$$
\mathrm{NS}\left(\mathcal{A}_{\eta}\right) \rightarrow \mathrm{NS}(A)
$$

contains $L_{1}, L_{2}$, where $\mathcal{A}_{\eta}$ is the generic fiber of $\mathcal{A}$ over $W^{\prime}$. In particular, every supersingular abelian surface $A$ admits a projective lift over $W$ such that $A$ is isogenous to the product of elliptic curves.

Proof. The proof is similar to that of Proposition 4.3. We first consider the case that $A$ is not supersingular. As in [50], let $\operatorname{Def}\left(A ; L_{1}, L_{2}\right)$ be the deformation functor parametrizing deformations of $A$ together with $L_{1}$ and $L_{2}$. For simplicity, we can assume that $L_{2}$ is a separable polarization. By deformation theory of abelian varieties, the formal deformation space $\operatorname{Def}(A)$ of $A$ over $W$ is isomorphic to

$$
\operatorname{Spf}\left(W\left[\left[t_{1}, t_{2}, t_{3}, t_{4}\right]\right]\right) .
$$

As inspired by Grothendieck and Mumford, each $L_{i}$ imposes one equation $f_{i}$ on $W\left[\left[t_{1}, t_{2}, t_{3}, t_{4}\right]\right](c f .[72, \S 2.3-2.4])$ and the forgetful functor $\operatorname{Def}\left(A ; L_{1}, L_{2}\right) \rightarrow$ $\operatorname{Def}(A)$ can be identified as the quotient map

$$
W\left[\left[t_{1}, t_{2}, t_{3}, t_{4}\right]\right] \rightarrow W\left[\left[t_{1}, t_{2}, t_{3}, t_{4}\right]\right] /\left(f_{1}, f_{2}\right) .
$$


Then it is easy to see that $\operatorname{Def}\left(A ; L_{1}, L_{2}\right)$ is formally smooth over $W$ (cf. $[72,2.4 .1]$ and [50, Proposition 4.1]). Since $W$ is Henselian, the $k$-valued point $\left(A ; L_{1}, L_{2}\right)$ extends to a $W$-valued point, giving a formal lifting. Moreover, the formal family is formally projective. By the Grothendieck Existence Theorem, this lift is therefore algebraizable as a projective scheme, as desired.

If $A$ is supersingular, as in [52, Lemma A.4], it suffices to show each triple $\left(A, L_{1}, L_{2}\right)$ is the specialization of an object $\left(A^{\prime}, L_{1}^{\prime}, L_{2}^{\prime}\right)$ of finite height along a local ring. In other words, we can deform an abelian surface in equi-characteristic to a non supersingular abelian surface. Consider the formal universal deformation space

$$
\Delta_{A}=\operatorname{Spec} k\left[\left[x_{1}, \ldots, x_{4}\right]\right]
$$

of $A$ over $k$. Similarly as above, each line bundle $L_{i}$ determines a divisor in $\Delta_{A}$. The complete local ring of $\Delta_{A}$ at $\left(X, L_{1}, L_{2}\right)$ is given by two equations $f_{1}, f_{2}$. Moreover, since $L_{1}$ is a separable polarization, the universal deformation $\left(\mathcal{A}, \mathcal{L}_{1}, \mathcal{L}_{2}\right)$ of the triple $\left(A, L_{1}, L_{2}\right)$ is

and it is algebraizable.

$$
\operatorname{Spf} k\left[\left[t_{1}, \ldots, t_{4}\right]\right] /\left(f_{1}, f_{2}\right)
$$

Now, it is known that the supersingular locus in the deformations of $A$ has dimension 1 ( $c f .[48])$. But the deformation space

$$
T:=\operatorname{Spec} k\left[\left[t_{1}, \ldots, t_{4}\right]\right] /\left(f_{1}, f_{2}\right)
$$

has dimension at least 2 and hence can not lie entirely in the supersingular locus. The generic point of $T$ parametrizes a triple $\left(A^{\prime}, L_{1}^{\prime}, L_{2}^{\prime}\right)$ with $A^{\prime}$ of finite height. This shows the claim and proves the assertion.

6.2. Generalized Kummer varieties. Let $A$ be an abelian surface over $k$ and $s: A^{[n+1]} \rightarrow A$ be the morphism induced by the additive structure on $A$, which is an isotrivial fibration. By definition, the generalized Kummer variety (see [11]) is its fiber over the origin:

$$
K_{n}(A):=s^{-1}\left(O_{A}\right),
$$

which is an integral variety of dimension $2 n$ with trivial dualizing sheaf. It will be an irreducible symplectic variety if it is smooth. We shall remark that different from the case of characteristic zero, the generalized Kummer varieties over positive characteristic fields can be singular and even non-normal (see [79] for some examples in characteristic 2). In [79], Schröer raised the question when $K_{n}(A)$ is smooth. Here we partially answer this question.

Proposition 6.5. $K_{n}(A)$ is a smooth irreducible symplectic variety if $p+n+1$.

Proof. We first show the smoothness. After the base change $(n+1)_{A}: A \rightarrow A$, we obtain a trivialization of $s: A^{[n+1]} \rightarrow A$, i.e. there is a cartesian commutative diagram

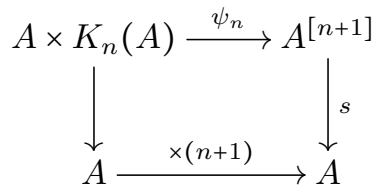

Since $p+n+1$, the map $\psi_{n}$ is étale. Hence $K_{n}(A)$ is smooth as well.

To show that $K_{n}(A)$ is irreducible symplectic, we proceed as in Proposition 4.5: 
by lifting $A$ to a base of characteristic zero, the simple connectedness and the degeneration of the Frölicher spectral sequence are obtained from their validity in characteristic zero by specialization.

6.3. Supersingularity and abelian motive. It turns out that the various notions of supersingularity coincide for generalized Kummer varieties, and they have supersingular abelian motives :

Proposition 6.6. Let $K_{n}(A)$ be a smooth generalized Kummer variety associated to an abelian surface $A$. The following conditions are equivalent:

(i) $A$ is supersingular.

(ii) $K_{n}(A)$ is $2^{\text {nd }}$-Artin supersingular;

(ii') $K_{n}(A)$ is fully Artin supersingular;

(iii) $K_{n}(A)$ is $2^{\text {nd }}$-Shioda supersingular;

(iii') $K_{n}(A)$ is fully Shioda supersingular;

(iv) The Chow motive of $K_{n}(A)$ is a supersingular abelian motive.

In this case, all the conclusions in the Supersingular Bloch-Beilinson Conjecture hold for $K_{n}(A)$.

Proof. Since the standard conjecture is known for generalized Kummer varieties, the notion of $2^{\text {nd }}$-Shioda supersingularity is independent of the cohomology theory used. Let us denote by $X$ the generalized Kummer variety $K_{n}(A)$ and $H^{i}(X)$ the crystalline cohomology $H^{i}(X / W)$, where $W$ is the Witt ring of the base field $k$.

Now we proceed the proof as following.

For $(i i) \Longrightarrow(i)$, since $H^{2}(X) \cong H^{2}(A) \oplus W(-1)$, the supersingularity of the $F$ crystal $H^{2}(X)$ implies the supersingularity of the crystal $H^{2}(A)$, which says that $A$ is a supersingular abelian surface.

The implications $\left(i i i^{\prime}\right) \Longrightarrow\left(i i^{\prime}\right)$ and $(i i i) \Longrightarrow(i i)$ are explained in Remark 2.5.

The implication $(i v) \Longrightarrow($ iii $)$ is Corollary $2.16(i i)$.

To complete the claimed equivalences, it remains to show that $(i) \Longrightarrow(i v)$. To this end, we use the following motivic decomposition of $K_{n}(A)$ ( $c f$. [21], [29, Theorem 7.9] and [90]) :

$$
\mathfrak{h}(X)(n) \cong \bigoplus_{\lambda \dashv(n+1)} \mathfrak{h}\left(A_{0}^{\lambda}\right)(|\lambda|)
$$

where $\lambda$ runs through all partitions of $n+1$; for a partition $\lambda=\left(\lambda_{1}, \cdots, \lambda_{l}\right),|\lambda|:=l$ denotes its length and

$$
A_{0}^{\lambda}:=\left\{\left(x_{1}, \cdots, x_{l}\right) \in A^{l} \mid \sum_{i} \lambda_{i} x_{i}=O_{A}\right\} .
$$

Observe that $A_{0}^{\lambda}$ is isomorphic, as algebraic varieties, to the disjoint union of $\operatorname{gcd}(\lambda)^{4}$ copies of the abelian variety $A^{l-1}$, where $\operatorname{gcd}(\lambda)$ is the greatest common divisor of $\lambda_{1}, \cdots, \lambda_{l}$. As a result, the motive of $X$ is a direct sum of the motives of some powers of $A$ with Tate twists, precisely:

$$
\mathfrak{h}(X)(n) \cong \bigoplus_{i} \mathfrak{h}\left(A^{l_{i}-1}\right)\left(l_{i}\right) .
$$

Since $A$ is supersingular, $\mathfrak{h}(X)$ is by definition a supersingular abelian motive.

Finally, the last assertion on supersingular Bloch-Beilinson conjecture follows from (iv) and Corollary 2.16. 
Remark 6.7. When $n>1$, the cohomology group $H^{3}\left(K_{n}(A), \mathcal{O}_{X}\right) \neq 0$ (it is of dimension $\left.\left(\begin{array}{c}2 n \\ 3\end{array}\right)+2 n-2\right)$. Therefore, we do not know the smoothness of its formal Brauer group and whether the $2^{\text {nd }}$-Artin supersingularity of $K_{n}(A)$ coincides with its Artin $\widehat{\mathrm{Br}}$-supersingularity. It is also very interesting to investigate the smoothness of formal Artin-Mazur functors of abelian varieties and its connection to supersingular abelian varieties.

6.4. Rational chain connectedness. We show the RCC conjecture for generalized Kummer varieties:

Proposition 6.8. Let $A$ be a supersingular abelian surface and $n$ a natural number, then the generalized Kummer variety $K_{n}(A)$ is rationally chain connected.

Proof. The main input is Shioda's theorem [82] that the Kummer K3 surface $K_{1}(A)$ is unirational. Let us first consider the singular model of $K_{n}(A)$, namely

$$
K_{n}^{\prime}(A):=A_{0}^{n+1} / \mathfrak{S}_{n+1},
$$

where $A_{0}^{n+1}:=\left\{\left(x_{0}, \cdots, x_{n}\right) \in A^{n+1} \mid \sum_{i} x_{i}=O\right\}$ equipped with the natural $\mathfrak{S}_{n+1^{-}}$ action by permutation. A typical element of $K_{n}^{\prime}(A)$ is thus denoted by $\left\{x_{0}, \cdots, x_{n}\right\}$.

Now we show that any two points of $K_{n}^{\prime}(A)$ can be connected by a chain of unirational surfaces $K_{1}^{\prime}(A)=A /-1$. Indeed, for any $\left\{x_{0}, \cdots, x_{n}\right\} \in K_{n}^{\prime}(A)$ (hence $\left.\sum_{i} x_{i}=0\right)$, let us explain how it is connected to $\{O, \cdots, O\}$. Firstly, the image of the map

$$
\begin{aligned}
\varphi_{1}: A /-1 & \rightarrow K_{n}^{\prime}(A) \\
t & \mapsto\{t,-t, O, \cdots, O\}
\end{aligned}
$$

connects $\{O, O, O, \cdots, O\}$ and $\left\{x_{0},-x_{0}, O, \cdots, O\right\}$. Next, one can choose any $u \in A$ such that $2 u=x_{0}$, then the surface

$$
\begin{aligned}
A /-1 & \rightarrow K_{n}^{\prime}(A) \\
t & \mapsto\left\{x_{0}, t-u,-t-u, O, \cdots, O\right\}
\end{aligned}
$$

connects the two points:

$$
\left\{x_{0},-x_{0}, O, \cdots, O\right\} \text { and }\left\{x_{0}, x_{1},-x_{0}-x_{1}, O, \cdots, O\right\}
$$

by taking $t=-u$ and $t=u+x_{1}$ respectively. Continuing this process $n$ times, we connect $\{O, \cdots, O\}$ to $\left\{x_{0}, \cdots, x_{n}\right\}$ in $K_{n}^{\prime}(A)$ by the unirational surfaces $K_{1}^{\prime}(A)$. In conclusion, $K_{n}^{\prime}(A)$ is rationally chain connected. At last, note that we have a birational morphism

$$
K_{n}(A) \rightarrow K_{n}^{\prime}(A)
$$

as the crepant resolution. As the exceptional divisors of (54) are rationally chain connected (see also Remark 7.9), it follows that $K_{n}(A)$ is rationally chain connected as well.

Remark 6.9. The existence of a unirational parametrization of $K_{n}^{\prime}(A)$ is unknown, even in the case $n=2$. 
6.5. Chow rings of supersingular generalized Kummer varieties. We establish the Section Conjecture and Beauville Splitting Conjecture for supersingular generalized Kummer varieties. Let $A$ be a supersingular abelian surface and $n \in \mathbb{N}$. Let $X=K_{n}(A)$ be a smooth generalized Kummer variety.

Proposition 6.10. The notation is as above. The rational Chow ring $\mathrm{CH}^{*}(X)_{\mathbb{Q}}$ contains a graded subalgebra $\mathrm{DCH}^{*}(X)$ containing all the Chern classes of $T_{X}$ and such that the composition $\mathrm{DCH}^{*}(X) \rightarrow \mathrm{CH}^{*}(X)_{\mathbb{Q}} \rightarrow \overline{\mathrm{CH}^{*}}(X)_{\mathbb{Q}}$ is an isomorphism.

Proof. By Proposition 3.22, it is enough to construct a marking (Definition 3.20) for $\mathfrak{h}(X)$ satisfying the condition $(\star)$ in Definition 3.21. Such a marking is given in $[30, \S 5.5 .2]$, which works also in positive characteristic.

Corollary 6.11. The Supersingular Beauville Splitting Conjecture holds for X.

Proof. This conjecture is a consequence of the combination of the Supersingular Bloch-Beilinson Conjecture and Supersingular Section Conjecture, which are established respectively in Proposition 6.6 and Proposition 6.10 for smooth generalized Kummer varieties.

\section{IrReducible symplectic Varieties of Generalized Kummer type}

Recall that an irreducible symplectic variety defined over the base field $k$ is called of generalized Kummer type if it is deformation equivalent to the generalized Kummer variety associated to an abelian surface (§6). Typical examples of such varieties are provided by the Albanese fibers of moduli spaces of stable sheaves on abelian surfaces. We give a similar construction in positive characteristic.

7.1. Moduli spaces of stable sheaves on abelian surfaces. Given an abelian surface $A$, the (algebraic) Mukai lattice $\widetilde{H}(A)$ is the free abelian group

$$
\widetilde{H}(A):=\mathbb{Z} \cdot \mathbb{1} \oplus \mathrm{NS}(A) \oplus \mathbb{Z} \cdot \omega
$$

endowed with the Mukai pairing $\langle-,-\rangle$ defined by

$$
\left\langle\left(v_{0}, v_{1}, v_{2}\right),\left(v_{0}^{\prime}, v_{1}^{\prime}, v_{2}^{\prime}\right)\right\rangle=v_{1} v_{1}^{\prime}-v_{0} v_{2}^{\prime}-v_{0}^{\prime} v_{2},
$$

where $\mathbb{1} \in H^{0}(A)$ is the fundamental class, $\omega \in H^{4}(A)$ is the class of a point and an element $r \cdot \mathbb{1}+L+s \cdot \omega$ is often denoted by $(r, L, s) \in \widetilde{H}(S)$. The Mukai vector of a sheaf $\mathcal{F}$ on $A$ is the same thing as its Chern character:

$$
v(\mathcal{F}):=\operatorname{ch}(\mathcal{F})=\left(\operatorname{rk}(\mathcal{F}), c_{1}(\mathcal{F}), \chi(\mathcal{F})\right) \in \widetilde{H}(A) .
$$

As in Definition 4.7 for K3 surfaces, a Mukai vector $v=\left(r, c_{1}, s\right) \in \widetilde{H}(A)$ is said to be coprime to $p$, if $p+r$ or $p+s$ or $p+c_{1} \cdot \mathrm{NS}(A)$. A polarization $H$ is said to be general with respect to $v$ if every $H$-semistable sheaf with Mukai vector $v$ is $H$-stable. Clearly, the numerical condition (17) implies the generality of $H$.

Given $v \in \widetilde{H}(A)$ and a general polarization $H$ with respect to $v$, we denote by $M_{H}(A, v)$ the moduli space of stable sheaves on $A$ with Mukai vector $v$. Associating to a sheaf its determinant and second Chern class induces the Albanese morphism

$$
\left(\operatorname{det}, c_{2}\right): M_{H}(A, v) \rightarrow \operatorname{Pic}^{0}(A) \times A,
$$

which is an isotrivial fibration. The Albanese fiber, denoted by $K_{H}(v)$, is an irreducible symplectic variety of generalized Kummer type of dimension $\langle v, v\rangle-2$, provided that it is smooth.

The following result is similar to Proposition 4.5 for K3 surfaces. 
Proposition 7.1. Let $A$ be a smooth abelian surface, $H$ a polarization and $v=$ $\left(r, c_{1}, s\right) \in \widetilde{H}(A)$ a primitive element such that $r>0$ and $\langle v, v\rangle \geq 2$. If $H$ is general with respect to $v$, then

(i) $M_{H}(A, v)$ is a smooth projective variety of dimension $\langle v, v\rangle+2$ over $k$. The fiber of the Albanese map

$$
M_{H}(A, v) \rightarrow A \times \widehat{A},
$$

denoted by $K_{H}(v)$, if smooth, is an irreducible symplectic variety of dimension $2 n:=\langle v, v\rangle-2$ and deformation equivalent to the generalized Kummer variety $K_{n}(A)$. In particular, it is the case when $p+n+1$.

(ii) When $\ell \neq p$, there is a canonical quadratic form on $H^{2}\left(K_{H}(v), \mathbb{Z}_{\ell}(1)\right)$ with values in $\mathbb{Z}\left[\frac{1}{\ell}\right]$. Let $v^{\perp}$ be the orthogonal complement of $v$ in the $\ell$-adic Mukai lattice of $A$. There is an injective isometry

$$
\theta_{v}: v^{\perp} \cap \widetilde{H}(A) \rightarrow \mathrm{NS}\left(K_{H}(v)\right),
$$

whose cokernel is a p-primary torsion group. Moreover, $|\operatorname{disc}(\mathrm{NS}(A))|$ divides $\mid \operatorname{disc}\left(\mathrm{NS}\left(K_{H}(v)\right) \mid\right.$.

(iii) If $p+n+1$, there is an isomorphism $v^{\perp} \otimes K \rightarrow H_{\text {cris }}^{2}\left(K_{H}(v) / W\right)_{K}$ as $F$ isocrystals.

Proof. Everything is known in characteristic zero ( $c f$. [62], [91, Theorem 0.1]). For fields of positive characteristic, the proof is the same as the case of K3 surfaces by the lifting argument, except for the smoothness of $K_{H}(v)$. For the smoothness, if suffices to note that there is also a trivialization of (56) via the base change $(n+1)_{A} \times \mathrm{id}: A \times \widehat{A}\left(c f\right.$. [91]), then $K_{H}(v)$ is smooth once $(n+1)_{A} \times$ id is etále.

Exactly as in Lemma 4.8, we do not have to worry about the numerical condition on the ample line bundle $H$ in Proposition 7.1, if the abelian surface is supersingular :

Lemma 7.2. Let $A$ be a supersingular abelian surface defined over an algebraically closed field of characteristic $p>0$. If $v \in \tilde{H}(A)$ is coprime to $p$, then any ample line bundle $H$ satisfies the numerical condition (17); hence is general with respect to $v$.

Proof. This is the same as the proof of Lemma 4.8.

Theorem 7.3 ([60, Theorem 0.2.11]). Let $k$ be an algebraically closed field of characteristic $p>0$. Let $A$ be a supersingular ablian surface over $k$. Let $v \in \tilde{H}(A)$ be a Mukai vector which is coprime to $p$. Let $H$ and $H^{\prime}$ be two ample line bundles on $A$. Then $M_{H}(A, v)$ is quasi-liftably birational to $M_{H^{\prime}}(A, v)$.

Proof. The birational equivalence is exactly the assertion of [60, Theorem 0.2.11]. To show this birational equivalence is liftable, we now prove it by lifting to characteristic 0 and its idea is very similar to Theorem 4.10 for the case of K3 surfaces. The only thing one has to be careful is that according to Proposition 6.4, we can only lift supersingular abelian surface with at most two independent line bundles.

Firstly, note that tensoring with a line bundle on $c_{1}$ would not change the moduli space, therefore, we can assume that $c_{1}=\left[H^{\prime \prime}\right]$ for some separably ample line bundle $H^{\prime \prime}$ after twisting some sufficiently separable ample line bundle to $c_{1}$. Using the coprime condition, Lemma 7.2 then ensure $\operatorname{gcd}\left(r, c_{1} \cdot H, s\right)=1$. Then we can lift the supersingular abelian surface with line bundles $H$ and $H^{\prime \prime}$. In this case, the Mukai vector $v$ can be lifted as well. So the same argument in Theorem 4.10 shows 
that $M_{H}(A, v)$ is birationally equivalent to $M_{H^{\prime \prime}}(A, v)$ via some liftably birational map by using the wall-crossing result [60, Theorem 0.2.11] of abelian surfaces in characteristic 0. Similarly, there is a quasi-liftably birational map $M_{H^{\prime}}(A, v) \rightarrow$ $M_{H^{\prime \prime}}(A, v)$ and the assertion follows.

7.2. Main results for generalized Kummer type moduli spaces. The goal of this subsection is to establish our conjectures, except the Unirationality Conjecture, for most of the smooth Albanese fibers of moduli spaces of stable sheaves on abelian surfaces (Theorem 1.5 and Corollary 1.6). Similarly to Proposition 4.13, we firstly establish them for all irreducible symplectic varieties which are quasi-liftably birational to smooth generalized Kummer varieties.

Proposition 7.4 (Birational generalized Kummer varieties). Let $X$ be an irreducible symplectic variety of dimension $2 n$ defined over an algebraically closed field $k$ of positive characteristic $p$. Suppose that $X$ is birational equivalent to the generalized Kummer variety $K_{n}(A)$ for some abelian surface $A$. Then the following are equivalent

(i) $A$ is supersingular.

(ii) $X$ is $2^{\text {nd }}$-Artin supersingular.

(iii) $X$ is $2^{\text {nd }}$-Shioda supersingular.

Moreover, if $X$ is quasi-liftably birational to $K_{n}(A)$, then $(i) \sim($ iii $)$ are also equivalent to any of the following

$\left(i i^{\prime}\right) X$ is fully Artin supersingular.

$\left(\right.$ iii' $\left.{ }^{\prime}\right) X$ is fully Shioda supersingular.

(iv) $X$ is rationally chain connected.

(v) $X$ has supersingular abelian motive.

In this case, all the conclusions in the Supersingular Bloch-Beilinson Conjecture, the Section Conjecture and the Supersingular Beauville Splitting Conjecture hold for $X$.

Proof. For the equivalences, use Remark 2.5, Theorem 2.8 and Corollary 2.16, we only need to establish the implications $(i i) \Rightarrow(i),(i) \Rightarrow(i v)$ and $(i) \Rightarrow(v)$.

For $(i i) \Rightarrow(i)$, as $X$ and $K_{n}(A)$ are $K$-equivalent, we have an isomorphism of isocrystals $H^{2}(X, K) \cong H^{2}\left(K_{n}(A), K\right)$, hence $K_{n}(A)$ is also $2^{n d}$-Artin supersingular. Hence $A$ is supersingular by Proposition 6.6.

For $(i) \Rightarrow(i v)$, given supersingular abelian surface $A$, its generalized Kummer variety $K_{n}(A)$ is rationally chain connected by Proposition 6.8. The the implication follows from the fact that rational chain connectedness is preserved under liftable birational transformations. Let us explain briefly this fact: according to the weak factorization theorem ([89], [2]), a liftable birational transformation can be viewed as the reduction of a sequence of blow-ups and blow-downs along smooth centers. As the exception divisors of the blow-ups and blow-downs are projective bundles over the center, the rational chain connectedness is preserved. It follows that $X$ is also rationally chain connected.

For $(i) \Rightarrow(v)$, the abelian surface $A$ being supersingular, we know from Proposition 6.6 that $K_{n}(A)$ has supersingular abelian motive. Hence so is $X$ because Proposition 3.24 implies that the Chow motives $\mathfrak{h}(X)$ and $\mathfrak{h}\left(K_{n}(A)\right)$ are isomorphic. All the equivalences are proved.

For the last assertion, the Supersingular Bloch-Beilinson Conjecture is a consequence of $(v)$ by Corollary 2.16. The Section Conjecture for $X$ follows from that 
for $K_{n}(A)$, which is established in Proposition 6.10, because Proposition 3.24 provides an algebraic correspondence inducing an isomorphism of $\mathbb{Q}$-algebras between $\mathrm{CH}^{*}(X)_{\mathbb{Q}}$ and $\mathrm{CH}^{*}\left(K_{n}(A)\right)_{\mathbb{Q}}$ which sends Chern classes of $T_{X}$ to the corresponding ones of $T_{K_{n}(A)}$. Finally, the Supersingular Beauville Splitting Conjecture follows by combining Supersingular Bloch-Beilinson Conjecture and Supersingular Section Conjecture.

Next, we turn to the study of moduli spaces of sheaves on abelian surfaces and their Albanese fibers in general. The key observation is again the existence of elliptic fibrations.

Theorem 7.5. Let $v=\left(r, c_{1}, s\right) \in \tilde{H}(A)$ be a Mukai vector, which is coprime to $p$. Then there exists an elliptic fibration $\pi: A \rightarrow E$ such that $\operatorname{gcd}\left(r, c_{1} \cdot f\right)=1$ where $f \in \mathrm{NS}(A)$ is the fiber class of $\pi$ and $E$ is an elliptic curve.

Proof. The existence of elliptic fibration is equivalent to say that there exists a square zero element in $\operatorname{NS}(A)$ satisfying the coprime condition (note that there is no $(-2)$ curves on an abelian surface). As the argument is exactly the same as in Theorem 4.15 (replace $\operatorname{NS}(S)$ by $\operatorname{NS}(A)$ everywhere and skip Step 0), we omit the details here.

Now we can relate a moduli space of generalized Kummer type to some generalized Kummer variety.

Theorem 7.6. Let $A$ be a supersingular abelian surface over an algebraically closed field $k$ of positive characteristic $p$. Let $v=\left(r, c_{1}, s\right) \in \widetilde{H}(A)$ be a Mukai vector coprime to $p$, with $r>0$ and $v^{2} \geq 2$, then there is a birational map

$$
M_{H}(A, v) \rightarrow \operatorname{Pic}^{0}\left(A^{\prime}\right) \times A^{\prime[n+1]}
$$

for some supersingular abelian surface $A^{\prime}$ and $n=\frac{v^{2}}{2}-1$. Moreover, when $K_{H}(v)$ is smooth, there is a quasi-liftably birational equivalence

$$
K_{H}(v) \rightarrow K_{n}\left(A^{\prime}\right) .
$$

Proof. Set $c_{1}=c_{1}(L)$ for some line bundle $L \in \operatorname{Pic}(A)$. Here we can assume $L$ is ample by tensoring a sufficiently ample line bundle to $L$. Let $E \in \operatorname{Pic}(A)$ be the line bundle which induces the elliptic fibration in Theorem 7.5. We claim that there exists a separable polarization

$$
H=L+n E \in \operatorname{Pic}(A)
$$

for some $n \in \mathbb{Z}^{\geq 0}$ up to a replacement of $L$ by $L+r L_{1}$ for any $L_{1} \in \operatorname{Pic}(A)$. To prove the claim, first note that it holds trivially if $p+c_{1}(L)^{2}$ or $p+c_{1}(L) \cdot c_{1}(E)$. Suppose $p \mid \operatorname{gcd}\left(c_{1}(L)^{2}, c_{1}(L) \cdot c_{1}(E)\right)$, then $p+r$ by our assumption. If $p \mid c_{1}(L) \cdot \mathrm{NS}(A)$, we can replace $L$ by $L+r H_{1}$, where $H_{1}$ is the principal polarization in Lemma 6.3. If $p+c_{1}(L) \cdot \mathrm{NS}(A)$, there must exist a square zero element $c_{1}\left(E^{\prime}\right) \in \mathrm{NS}(A)$ such that $p+c_{1}(L) \cdot c_{1}\left(E^{\prime}\right)$. Then one can replace $L$ by $L+r E^{\prime}$ which satisfies the condition as desired.

Now, one can lift the triple $(A, H, E)$ to characteristic 0 by Proposition 6.4. Using the same argument as in Theorem 4.16, one can deduce (57) by using Theorem 7.3, Theorem 4.14 and the specialization argument. To see that $A^{\prime}$ is supersingular, note that $M_{H}(A, v)$ and $\operatorname{Pic}^{0}\left(A^{\prime}\right) \times A^{\prime[n+1]}$, being birational, must have isomorphic Albanese varieties:

$$
\hat{A} \times A \simeq \hat{A}^{\prime} \times A^{\prime},
$$


which are supersingular since $A$ is. Hence so is $A^{\prime}$.

Furthermore, the birational equivalence (57) yields the following diagram

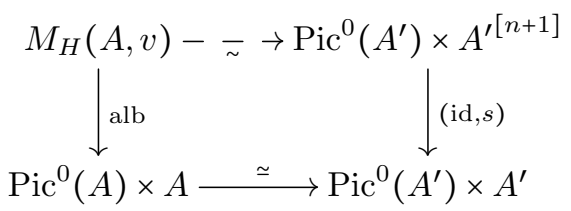

where the vertical maps are the isotrivial albanese fibrations, the commutativity of the diagram follows from the functoriality of Albanese map and the bottom map is an isomorphism, thanks to the general fact that a birational equivalence between two smooth projective varieties induces an isomorphism between their Albanese varieties. Now take any non-empty open subsets $U$ in $M_{H}(v)$ and $V$ in $\operatorname{Pic}^{0}\left(A^{\prime}\right) \times\left(A^{\prime}\right)^{[n+1]}$ which are identified under the birational equivalence (57). By restricting to general fibers of the two isotrivial Albanese fibrations, this induces an isomorphism of a non-empty open subset of $K_{H}(v)$ to an open subset of $K_{n}\left(A^{\prime}\right)$, that is, a birational equivalence between them.

Remark 7.7. Similarly as in Remark 4.9, the coprime assumption on $v$ is automatically satisfied if $p+\frac{1}{2} \operatorname{dim} X+1$.

Remark 7.8. The abelian surface $A^{\prime}$ in Theorem 7.6 is derived equivalent to $A$. When $A$ is the product of elliptic curves, we know that $A^{\prime}$ has to be isomorphic to $A$ ( $c f$. [36]). In general, the supersingular abelian surface $A^{\prime}$ is expected to be isomorphic to either $A$ or its dual $A^{\vee}$ (cf. [37]).

Proof of Theorem 1.5 and Corollary 1.6. Combine Proposition 7.4 with Theorem 7.6.

Remark 7.9. Another way to see that the rational chain connectedness is preserved under the birational transformation (58) without lifting to characteristic zero is to consider the unirational surfaces covering of $K_{n}\left(A^{\prime}\right)$ constructed in Proposition 6.8. We left it to the interested readers.

7.3. A remark on the unirationality. Theorem 7.6 also provides us an approach to the Unirationality Conjecture 3.15 for generalized Kummer type varieties $K_{H}(A, v)$.

Corollary 7.10. Notation and assumption are as in Theorem 7.6. If $A$ is supersingular, then $K_{H}(A, v)$ is unirational if the generalized Kummer variety $K_{n}(E \times E)$ is unirational for some supersingular elliptic curve $E$, where $n=\frac{v^{2}}{2}-1$.

Proof. By Theorem 7.6, $K_{H}(A, v)$ is birational to $K_{n}\left(A^{\prime}\right)$ for some supersingular abelian surface $A^{\prime}$. By [73, Theorem 4.2], for any supersingular elliptic curve $E, A^{\prime}$ and $E \times E$ are isogenous. Therefore it suffices to see that for two isogenous abelian surfaces $A_{1}$ and $A_{2}$, the generalized Kummer varieties $K_{n}\left(A_{1}\right)$ and $K_{n}\left(A_{2}\right)$ are dominated by each other. To this end, we remark that $B_{1}:=\operatorname{ker}\left(A_{1}^{n+1} \stackrel{+}{\rightarrow} A_{1}\right)$ has an isogeny to $B_{2}:=\operatorname{ker}\left(A_{2}^{n+1} \stackrel{+}{\rightarrow} A_{2}\right)$ which is compatible with the natural $\mathfrak{S}_{n+1}$ actions. Therefore $K_{n}^{\prime}\left(A_{1}\right):=B_{1} / \mathfrak{S}_{n+1}$ has a dominant map to $K_{n}^{\prime}\left(A_{2}\right):=B_{2} / \mathfrak{S}_{n+1}$. Hence $K_{n}\left(A_{1}\right)$ dominates $K_{n}\left(A_{2}\right)$. By symmetry, they dominate each other. 
For each $p$ and $n$, Corollary 7.10 allows us to reduce Conjecture 3.14 for moduli spaces of generalized Kummer type to a very concrete question:

Question 7.11. Let $E$ be a supersingular elliptic curve. Is the generalized Kummer variety $K_{n}(E \times E)$ unirational?

\section{REFERENCES}

[1] Revêtements étales et groupe fondamental (SGA 1), volume 3 of Documents Mathématiques (Paris) [Mathematical Documents (Paris)]. Société Mathématique de France, Paris, 2003. Séminaire de géométrie algébrique du Bois Marie 1960-61. [Algebraic Geometry Seminar of Bois Marie 1960-61], Directed by A. Grothendieck, With two papers by M. Raynaud, Updated and annotated reprint of the 1971 original [Lecture Notes in Math., 224, Springer, Berlin; MR0354651 (50 \#7129)]. 30

[2] Dan Abramovich, Kalle Karu, Kenji Matsuki, and Jarosł aw Wł odarczyk. Torification and factorization of birational maps. J. Amer. Math. Soc., 15(3):531-572, 2002. 49

[3] Yves André. Une introduction aux motifs (motifs purs, motifs mixtes, périodes), volume 17 of Panoramas et Synthèses [Panoramas and Syntheses]. Société Mathématique de France, Paris, 2004. 11, 12

[4] Yves André. Motifs de dimension finie (d'après S.-I. Kimura, P. O’Sullivan...). Astérisque, (299):Exp. No. 929, viii, 115-145, 2005. Séminaire Bourbaki. Vol. 2003/2004. 15

[5] M. Artin. Supersingular K3 surfaces. Ann. Sci. École Norm. Sup. (4), 7:543-567 (1975), 1974. 3, 4, 19, 20, 26, 39

[6] M. Artin and B. Mazur. Formal groups arising from algebraic varieties. Ann. Sci. École Norm. Sup. (4), 10(1):87-131, 1977. 3, 19

[7] Arend Bayer and Emanuele Macrì. MMP for moduli of sheaves on K3s via wall-crossing: nef and movable cones, Lagrangian fibrations. Invent. Math., 198(3):505-590, 2014. 18, 32, 33, 34,35

[8] Arend Bayer and Emanuele Macrì. Projectivity and birational geometry of Bridgeland moduli spaces. J. Amer. Math. Soc., 27(3):707-752, 2014. 18

[9] Arnaud Beauville. Variétés de Prym et jacobiennes intermédiaires. Ann. Sci. École Norm. Sup. (4), 10(3):309-391, 1977. 12

[10] Arnaud Beauville. Quelques remarques sur la transformation de Fourier dans l'anneau de Chow d'une variété abélienne. In Algebraic geometry (Tokyo/Kyoto, 1982), volume 1016 of Lecture Notes in Math., pages 238-260. Springer, Berlin, 1983. 14, 17, 23

[11] Arnaud Beauville. Variétés Kähleriennes dont la première classe de Chern est nulle. J. Differential Geom., 18(4):755-782 (1984), 1983. 4, 18, 44

[12] Arnaud Beauville. Sur l'anneau de Chow d'une variété abélienne. Math. Ann., 273(4):647651, 1986. 14, 17, 23

[13] Arnaud Beauville. On the splitting of the Bloch-Beilinson filtration. In Algebraic cycles and motives. Vol. 2, volume 344 of London Math. Soc. Lecture Note Ser., pages 38-53. Cambridge Univ. Press, Cambridge, 2007. 6, 7, 22, 23

[14] Arnaud Beauville and Ron Donagi. La variété des droites d'une hypersurface cubique de dimension 4. C. R. Acad. Sci. Paris Sér. I Math., 301(14):703-706, 1985. 18

[15] Spencer Bloch. Lectures on algebraic cycles, volume 16 of New Mathematical Monographs. Cambridge University Press, Cambridge, second edition, 2010. 16

[16] Daniel Bragg and Max Lieblich. Twistor spaces for supersingular K3 surfaces. arXiv:1804.07282., 2018. 3, 21, 29, 30, 33, 38, 39, 40, 41

[17] Tom Bridgeland. Fourier-Mukai transforms for elliptic surfaces. J. Reine Angew. Math., 498:115-133, 1998. 34, 35

[18] François Charles. The Tate conjecture for $K 3$ surfaces over finite fields. Invent. Math., 194(1):119-145, 2013. 3, 20, 26

[19] François Charles. Birational boundedness for holomorphic symplectic varieties, Zarhin's trick for K3 surfaces, and the Tate conjecture. Ann. of Math. (2), 184(2):487-526, 2016. 3, 4, 26, $27,28,29,30,39$

[20] Mark Andrea A. de Cataldo and Luca Migliorini. The Chow groups and the motive of the Hilbert scheme of points on a surface. J. Algebra, 251(2):824-848, 2002. 34 
[21] Mark Andrea A. de Cataldo and Luca Migliorini. The Chow motive of semismall resolutions. Math. Res. Lett., 11(2-3):151-170, 2004. 45

[22] A.J. De Jong. On a result of Artin. available at www.math.columbia.ed/d̃ejong/, 2011. 20

[23] Pierre Deligne. La conjecture de Weil. I. Inst. Hautes Études Sci. Publ. Math., (43):273-307, 1974. 10

[24] Pierre Deligne. La conjecture de Weil. II. Inst. Hautes Études Sci. Publ. Math., (52):137-252, 1980. 10

[25] Pierre Deligne and Luc Illusie. Relèvements modulo $p^{2}$ et décomposition du complexe de de Rham. Invent. Math., 89(2):247-270, 1987. 30

[26] Christopher Deninger and Jacob Murre. Motivic decomposition of abelian schemes and the Fourier transform. J. Reine Angew. Math., 422:201-219, 1991. 14

[27] Najmuddin Fakhruddin. On the Chow groups of supersingular varieties. Canad. Math. Bull., 45(2):204-212, 2002. 5, 6, 9, 13, 15, 16, 17

[28] Jean-Marc Fontaine and William Messing. p-adic periods and $p$-adic étale cohomology. In Current trends in arithmetical algebraic geometry (Arcata, Calif., 1985), volume 67 of Contemp. Math., pages 179-207. Amer. Math. Soc., Providence, RI, 1987. 30

[29] Lie Fu, Zhiyu Tian, and Charles Vial. Motivic hyperKähler resolution conjecture: I. generalized Kummer varieties. To appear in Geometry and Topology, arXiv:1608.04968, 2018. 45

[30] Lie Fu and Charles Vial. Distinguished cycles on varieties with motive of abelian type and the Section Property. To appear in Journal of Algebraic Geometry, arXiv:1709.05644, 2018. $6,22,23,24,47$

[31] William Fulton. Intersection theory, volume 2 of Ergebnisse der Mathematik und ihrer Grenzgebiete. 3. Folge. A Series of Modern Surveys in Mathematics [Results in Mathematics and Related Areas. 3rd Series. A Series of Modern Surveys in Mathematics]. Springer-Verlag, Berlin, second edition, 1998. 26

[32] William Fulton and Joe Harris. Representation theory, volume 129 of Graduate Texts in Mathematics. Springer-Verlag, New York, 1991. A first course, Readings in Mathematics. 15

[33] B. Brent Gordon and Kirti Joshi. Griffiths groups of supersingular abelian varieties. Canad. Math. Bull., 45(2):213-219, 2002. 17

[34] Frank Gounelas and Ariyan Javanpeykar. Invariants of Fano varieties in families. arXiv:1703.05735, 2017. 12

[35] M. Gross, D. Huybrechts, and D. Joyce. Calabi-Yau manifolds and related geometries. Universitext. Springer-Verlag, Berlin, 2003. Lectures from the Summer School held in Nordfjordeid, June 2001. 4

[36] Katrina Honigs, Luigi Lombardi, and Sofia Tirabassi. Derived equivalences of canonical covers of hyperelliptic and Enriques surfaces in positive characteristic. arXiv:1606.02094., 2016. 51

[37] Shinobu Hosono, Bong H. Lian, Keiji Oguiso, and Shing-Tung Yau. Kummer structures on K3 surface: an old question of T. Shioda. Duke Math. J., 120(3):635-647, 2003. 51

[38] D. Huybrechts. Fourier-Mukai transforms in algebraic geometry. Oxford Mathematical Monographs. The Clarendon Press, Oxford University Press, Oxford, 2006. 30

[39] Daniel Huybrechts. Compact hyper-Kähler manifolds: basic results. Invent. Math., 135(1):63113, 1999. 4, 18, 25, 28

[40] Luc Illusie. Complexe de de Rham-Witt et cohomologie cristalline. Ann. Sci. École Norm. Sup. (4), 12(4):501-661, 1979. 38

[41] Nicholas M. Katz and William Messing. Some consequences of the Riemann hypothesis for varieties over finite fields. Invent. Math., 23:73-77, 1974. 10

[42] Yujiro Kawamata. D-equivalence and K-equivalence. J. Differential Geom., 61(1):147-171, 2002. 33

[43] Wansu Kim and Keerthi Madapusi Pera. 2-adic integral canonical models. Forum Math. Sigma, 4:e28, 34, 2016. 3, 26

[44] Guido Kings. Higher regulators, Hilbert modular surfaces, and special values of $L$-functions. Duke Math. J., 92(1):61-127, 1998. 14

[45] Klaus Künnemann. On the Chow motive of an abelian scheme. In Motives (Seattle, WA, 1991), volume 55 of Proc. Sympos. Pure Math., pages 189-205. Amer. Math. Soc., Providence, RI, 1994. 14

[46] Nikon Kurnosov, Andrey Soldatenkov, and Misha Verbitsky. Kuga-Satake construction and cohomology of hyperkähler manifolds. preprint, arXiv:1703.07477, 2017. 22 
[47] Adrian Langer. Semistable sheaves in positive characteristic. Ann. of Math. (2), 159(1):251276, 2004. 28, 32

[48] Ke-Zheng Li and Frans Oort. Moduli of supersingular abelian varieties, volume 1680 of Lecture Notes in Mathematics. Springer-Verlag, Berlin, 1998. 44

[49] Max Lieblich. Moduli of twisted sheaves. Duke Math. J., 138(1):23-118, 2007. 38, 39

[50] Max Lieblich and Davesh Maulik. A note on the cone conjecture for K3 surfaces in positive characteristic. arXiv:1102.3377., 2011. 38, 43, 44

[51] Max Lieblich, Davesh Maulik, and Andrew Snowden. Finiteness of K3 surfaces and the Tate conjecture. Ann. Sci. Éc. Norm. Supér. (4), 47(2):285-308, 2014. 38, 39

[52] Max Lieblich and Martin Olsson. Fourier-Mukai partners of K3 surfaces in positive characteristic. Ann. Sci. Éc. Norm. Supér. (4), 48(5):1001-1033, 2015. 27, 44

[53] Christian Liedtke. Supersingular K3 surfaces are unirational. Inventiones Mathematicae, 200:979-1014, 2015. 3, 21, 27, 33, 35, 43

[54] Christian Liedtke. Lectures on supersingular K3 surfaces and the crystalline Torelli theorem. In K3 surfaces and their moduli, volume 315 of Progr. Math., pages 171-235. Birkhäuser/Springer, [Cham], 2016. 3, 26

[55] Keerthi Madapusi Pera. The Tate conjecture for K3 surfaces in odd characteristic. Invent. Math., 201(2):625-668, 2015. 3, 26

[56] Ju. I. Manin. Theory of commutative formal groups over fields of finite characteristic. Uspehi Mat. Nauk, 18(6 (114)):3-90, 1963. 10, 19

[57] Eyal Markman. Integral generators for the cohomology ring of moduli spaces of sheaves over Poisson surfaces. Adv. Math., 208(2):622-646, 2007. 30

[58] T. Matsusaka and D. Mumford. Two fundamental theorems on deformations of polarized varieties. Amer. J. Math., 86:668-684, 1964. 32

[59] Davesh Maulik. Supersingular K3 surfaces for large primes. Duke Math. J., 163(13):23572425, 2014. With an appendix by Andrew Snowden. 3

[60] Hiroki Minamide, Shintarou Yanagida, and Kōta Yoshioka. The wall-crossing behavior for Bridgeland's stability conditions on abelian and K3 surfaces. arXiv:1106.5217. J. Reine Angew. Math. to appear (available online, DOT: 10.1515/crelle-2015-0010), 2015. 33, 34, 48,49

[61] Matthew Morrow. A Variational Tate conjecture in crystalline cohomology. To appear in J. Eur. Math. Soc. (JEMS), arXiv:1408.6783., 2018. 4, 20

[62] Shigeru Mukai. Symplectic structure of the moduli space of sheaves on an abelian or $K 3$ surface. Invent. Math., 77(1):101-116, 1984. 18, 28, 29, 48

[63] David Mumford. Rational equivalence of 0-cycles on surfaces. J. Math. Kyoto Univ., 9:195204, 1968. 3, 12

[64] J. P. Murre. Applications of algebraic $K$-theory to the theory of algebraic cycles. In Algebraic geometry, Sitges (Barcelona), 1983, volume 1124 of Lecture Notes in Math., pages 216-261. Springer, Berlin, 1985. 12, 13

[65] Peter Norman and Frans Oort. Moduli of abelian varieties. Ann. of Math. (2), 112(3):413-439, 1980. 43

[66] N. O. Nygaard. The Tate conjecture for ordinary K3 surfaces over finite fields. Invent. Math., 74(2):213-237, 1983. 3

[67] Niels Nygaard and Arthur Ogus. Tate's conjecture for $K 3$ surfaces of finite height. Ann. of Math. (2), 122(3):461-507, 1985. 3

[68] Kieran G. O'Grady. The weight-two Hodge structure of moduli spaces of sheaves on a $K 3$ surface. J. Algebraic Geom., 6(4):599-644, 1997. 18, 28, 29

[69] Kieran G. O'Grady. Desingularized moduli spaces of sheaves on a K3. J. Reine Angew. Math., 512:49-117, 1999. 18

[70] Kieran G. O'Grady. A new six-dimensional irreducible symplectic variety. J. Algebraic Geom., 12(3):435-505, 2003. 18

[71] Arthur Ogus. Supersingular K3 crystals. In Journées de Géométrie Algébrique de Rennes (Rennes, 1978), Vol. II, volume 64 of Astérisque, pages 3-86. Soc. Math. France, Paris, 1979. 27

[72] Frans Oort. Finite group schemes, local moduli for abelian varieties, and lifting problems. Compositio Math., 23:265-296, 1971. 43, 44

[73] Frans Oort. Subvarieties of moduli spaces. Invent. Math., 24:95-119, 1974. 14, 15, 51 
[74] Peter O'Sullivan. Algebraic cycles on an abelian variety. J. Reine Angew. Math., 654:1-81, 2011. 23

[75] Vladimir Platonov and Andrei Rapinchuk. Algebraic groups and number theory, volume 139 of Pure and Applied Mathematics. Academic Press, Inc., Boston, MA, 1994. Translated from the 1991 Russian original by Rachel Rowen. 37

[76] Ulrike Rieß. On the Chow ring of birational irreducible symplectic varieties. Manuscripta Math., 145(3-4):473-501, 2014. 25

[77] A. N. Rudakov and I. R. Šafarevič. Supersingular $K 3$ surfaces over fields of characteristic 2. Izv. Akad. Nauk SSSR Ser. Mat., 42(4):848-869, 1978. 3, 21, 26, 27, 31, 33, 41, 42

[78] Hiroshi Saito. Abelian varieties attached to cycles of intermediate dimension. Nagoya Math. J., 75:95-119, 1979. 16

[79] Stefan Schröer. The Hilbert scheme of points for supersingular abelian surfaces. Ark. Mat., 47(1):143-181, 2009. 18, 44

[80] Ichiro Shimada. Supersingular $K 3$ surfaces in odd characteristic and sextic double planes. Math. Ann., 328(3):451-468, 2004. 26, 42

[81] Tetsuji Shioda. An example of unirational surfaces in characteristic p. Math. Ann., 211:233236, 1974. 3, 4

[82] Tetsuji Shioda. Some results on unirationality of algebraic surfaces. Math. Ann., 230(2):153168, 1977. 46

[83] John T. Tate. Algebraic cycles and poles of zeta functions. In Arithmetical Algebraic Geometry (Proc. Conf. Purdue Univ., 1963), pages 93-110. Harper \& Row, New York, 1965. 3

[84] G. van der Geer and T. Katsura. On the height of Calabi-Yau varieties in positive characteristic. Doc. Math., 8:97-113 (electronic), 2003. 11

[85] Claire Voisin. Théorie de Hodge et géométrie algébrique complexe, volume 10 of Cours Spécialisés [Specialized Courses]. Société Mathématique de France, Paris, 2002. 12

[86] Claire Voisin. Intrinsic pseudo-volume forms and $K$-correspondences. In The Fano Conference, pages 761-792. Univ. Torino, Turin, 2004. 33

[87] Claire Voisin. On the Chow ring of certain algebraic hyper-Kähler manifolds. Pure Appl. Math. Q., 4(3, part 2):613-649, 2008. 6

[88] Chin-Lung Wang. $K$-equivalence in birational geometry and characterizations of complex elliptic genera. J. Algebraic Geom., 12(2):285-306, 2003. 33

[89] Jarosł aw Wł odarczyk. Toroidal varieties and the weak factorization theorem. Invent. Math., 154(2):223-331, 2003. 49

[90] Ze Xu. Algebraic cycles on a generalized Kummer variety. Int. Math. Res. Not. IMRN, (3):932-948, 2018. 45

[91] Kōta Yoshioka. Moduli spaces of stable sheaves on abelian surfaces. Math. Ann., 321(4):817884, 2001. 18, 48

[92] Kōta Yoshioka. Moduli spaces of twisted sheaves on a projective variety. In Moduli spaces and arithmetic geometry, volume 45 of Adv. Stud. Pure Math., pages 1-30. Math. Soc. Japan, Tokyo, 2006. 29, 39

[93] Kōta Yoshioka. Moduli spaces of stable sheaves on Enrique surfaces. Preprint, arXiv:1602.06914, 2016. 34

Institut Camille Jordan, Université Claude Bernard Lyon 1, 43 Boulevard du 11 novembre 1918, 69622 Villeurbanne Cedex, France

E-mail address: fu@math.univ-lyon1.fr

Shanghai Center for Mathematical Science, Fudan University, 2005 Songhu Road, 20438 Shanghai, China

E-mail address: zhiyuan_li@fudan.edu.cn 